\title{
A summary of strontium and oxygen isotope variation in human tooth enamel excavated from Britain over the last 6000 years.
}

\author{
J. A. Evans ${ }^{1}$, C. A. Chenery ${ }^{1}$ and J. Montgomery ${ }^{2}$ \\ 1) NIGL, BGS, Keyworth, Nottingham, NG12 5GG, UK. \\ 2) Department of Archaeology, Durham University, South Road Durham, DH1 3LE \\ UK
}

Contact author J.A Evans. je@bgs.ac.uk

Keywords: strontium, oxygen, isotopes, enamel, Britain, migration.

\begin{abstract}
This paper presents a compilation of strontium and oxygen isotope data from human tooth enamel that has been produced at NERC Isotope Geosciences Laboratory over the last c. 15 years. These many and often small studies are here combined to provide an overview of data from Britain. The strontium isotope composition ranges between 0.7078 and 0.7165 (excluding individuals deemed to be of non-British origin). The median $\mathrm{Sr}$ concentration is $84 \mathrm{ppm}$ but there is a vector of increasing Sr concentrations related to seawater strontium isotope composition that is seen in individuals predominantly from the west coast of Scotland attributed to the used of kelp as a fertilizer. The oxygen isotope data is normally distributed with a mean value of $17.7 \%$ $\pm 1.4 \%$ ( $2 \mathrm{SD} n=615)$. Two sub-populations of local individuals have been identified that provide control groups for human enamel values from the eastern side of Britain where there are lower rainfall levels: $17.2 \% \pm \pm 1.26 \%,(2 \mathrm{SD}, \mathrm{n}=83)$ and western area of Britain where rainfall levels are higher $=18.2 \% 0 \pm 0.98 \%$, $(2 \mathrm{SD}, \mathrm{n}=41)$. These data make it possible to make direct comparisons of population means between burial populations and the control dataset to assess commonality of origin.
\end{abstract}

\section{Introduction}

Since the 1990's the isotope compositions of strontium and oxygen have been used by archaeologists to determine residential origins of our human ancestors. The basic premise is "you are what you eat and drink" and that the isotope values for strontium and oxygen in body tissues, such as bone and enamel bioapatite, can be linked through the biosphere to the geologic (strontium) and climatic (oxygen) environment in which an individual lived. Over the past $c .15$ years the NERC Isotope Geosciences Laboratory (NIGL) has analysed $c$. 1000 archaeological tooth enamel samples. The aim of this paper is to use this data to: 1) 
assess the overall structure and range of human isotope data from Britain over the past 6000 years; 2) compare these data with independent assessments of such ranges; 3) define limiting values and datasets in order to provide a set of references and methods that can be used for comparative studies in archaeological, forensic and modern migratory studies.

The basic principles of using strontium and oxygen isotope analysis of tooth enamel to comment on the childhood origins of humans is well documented ${ }^{1}$. In essence, strontium isotope composition provides links to the land where food was grown or grazed, and oxygen isotope composition is linked to the source of drinking water ${ }^{2-4}$.

\section{Materials and Methods}

The data for this study come from a large number of published sources ${ }^{4-25}$ and from a number of unpublished and grey literature studies. The tooth enamel analysed is from individuals excavated at 74 British archaeological sites dating from the Neolithic to the $19^{\text {th }}$ century A.D. and from across much of Britain. These samples were not collected systematically and this has inevitably introduced certain geographic biases, such that East Anglia, SW England and Wales are poorly represented (Figure 1). The reported dataset has not been filtered and includes individuals that are unlikely to be of British origin.

To preserve internal consistency and avoid inter-laboratory method bias, only data produced at NIGL is used for this study. With the exception of oxygen isotope data for West Heslerton and Monkton, all ${ }^{87} \mathrm{Sr} /{ }^{86} \mathrm{Sr}$ and $\delta^{18} \mathrm{O}$ values were obtained using the methods given in Evans et al. (2006). Oxygen isotope analysis for West Heslerton and Monkton samples was carried out by laser fluorination ${ }^{26}$. The data are presented in Appendix 1, location and references to published sources in Appendix 2. The Levinson ${ }^{2}$ drinking water equation modified by Chenery et al. $2010^{4}$ and equation 4 from Daux et al. $2008^{3}$ are the phosphate oxygen to drinking water conversions used in this paper when discussing the relationship between oxygen isotope composition measured in tooth enamel and 'drinking water'.

\section{Strontium concentration range and isotope composition found in individuals excavated from Britain.}

Knowing the range of biosphere isotope values that can be found within Great Britain gives us an important tool for identifying individuals who fall outside those limits and hence can be defined as non-British. To do this we need to characterise British variation, but this is not straightforward. It is helpful to consider Scotland separately from England and Wales because it has a different geological history and thus different geological and strontium 
isotope characteristics. Britain is divided, geologically, along a line referred to as the "Iapetus Suture". This geological junction runs from what is now the Solway Firth north east across England, roughly parallel with the England/Scotland border. This marks the contact between two different geological terrains: Scotland, derived from an ancient continent with parts still present in NW Scotland over 2000 million year old, known as Laurentia; and England and Wales, which were part of a micro-continent called Avalonia which has a maximum age of only c. 700 million years ${ }^{27}$. Scotland also contains the youngest rocks in Britain, the Tertiary Basalts, and hence has the potential for a much wider range of strontium isotope biosphere compositions compared with England and Wales.

The strontium concentrations and isotope compositions of the human tooth enamel available from Britain (Scotland, England and Wales combined) are presented in Figure 2. The mean and standard deviation of the concentrations and isotope ratios are $105 \pm 138 \mathrm{ppm}$ (2SD, $\mathrm{n}=614)$ and $0.7099 \pm 0.0026(2 \mathrm{SD}, \mathrm{n}=614)$, respectively. The isotope compositions range between a minimum value of 0.7064 and a maximum of 0.7205 . However, there is independent evidence to suggest that neither of these individuals is of British origin ${ }^{8,10,28}$ and consequently they have been excluded, reducing the range for Britain to $0.7078-0.7165$. Maximum ${ }^{87} \mathrm{Sr} /{ }^{86} \mathrm{Sr}$ values. Because the geology of Scotland is much older than that of most of England and Wales, and biosphere values up to 0.72 have been obtained from Scottish vegetation $^{29}$, it is to be expected that individuals with the highest values will be found in Scotland. However, bone preservation is poor in upland acidic soils, which means that skeletal survival is low in the Scottish Highlands and there is limited data available to provide a reliable estimate. The highest human value from Scotland is ${ }^{87} \mathrm{Sr} /{ }^{86} \mathrm{Sr}=0.7165(\mathrm{PH} \mathrm{SK} 71)$. The best estimate for a maximum human value for England and Wales comes from the average provided by the analysis of a historically defined group of 16 local individuals recovered from the medieval cemetery at Hereford Cathedral. With a highest measured value of 0.7140 , the group provides a mean ${ }^{87} \mathrm{Sr} /{ }^{86} \mathrm{Sr}$ of $0.7124 \pm 0.0022(2 \mathrm{SD}, \mathrm{n}=16)$. A high value of 0.7142 was also obtained in England from a single individual from the Blackfriars cemetery in Gloucester, less than 30 miles away from Hereford ${ }^{5}$, but as a single individual there is more uncertainty about its likely geographic origin.

We have, therefore, a local maximum of 0.7140 from Hereford as the current highest human value from England and Wales and a possible highest value from Scotland of 0.7165. 


\section{Minimum values}

Unlike the maximum human isotope values, which show a high degree of dispersal, there is a relatively clear minimum baseline for England and Wales of ${ }^{87} \mathrm{Sr} /{ }^{86} \mathrm{Sr}=0.7078$, which originates within the Cretaceous Chalk terrain. In Scotland, the Tertiary volcanic rocks are the primary source of low ${ }^{87} \mathrm{Sr} /{ }^{86} \mathrm{Sr}$ values and the lowest recorded human individual comes from Cnip in the Outer Hebrides with a ${ }^{87} \mathrm{Sr} /{ }^{86} \mathrm{Sr}$ value of 0.7078 (Cnip-D, Montgomery et al., 2003); this individual is not of Outer Hebridean origin but has a value that could be derived from the basalt terrains of the inner Hebridean islands such as Skye.

The range of strontium isotope ratios in human enamel is compared with the range of values measured in the British biosphere ${ }^{30}$ (Figure 3). These box plots highlight two features: 1) that the medians $0.7096(, n=614)$ and $0.7097(n=313)$ are similar for enamel and biosphere respectively with both having a tail of values towards the higher ranges of values; 2) that the human data is less diverse (inter-quartile rang 0.0014) than the biosphere data (inter-quartile range $=0.0024)$ which is largely based on plant samples. This can be explained as a result of humans ingesting food from a variety of origins and hence creating an average composition of their intake, thereby reducing the range of values in comparison to populations lower down the food chain ${ }^{31}$.

\section{The effect of environmental factors on the strontium concentrations}

The majority of samples have strontium concentrations below $150 \mathrm{ppm}$ (median value of data is $84 \mathrm{ppm}$ ), but a significant number have values up to $417 \mathrm{ppm}$ (Cnip G). A notable feature of the strontium system is the large proportion of individuals displaying high enamel strontium concentrations that are accompanied by a ${ }^{87} \mathrm{Sr} /{ }^{86} \mathrm{Sr}$ value resembling that of modern seawater. The majority of individuals, with elevated strontium concentration and seawater strontium isotope compositions, were excavated from island or coastal areas of Scotland that are associated with machair soils. These are calcium carbonate-rich soils, developed in marine shell debris, and often constitute the most fertile areas of these islands. There is no evidence that high enamel strontium concentrations are related fundamentally either to diagenesis within a carbonate-rich burial environment, or to the exploitation of a carbonaterich soil resource during life; that is, elevated concentrations are not seen in other high carbonate terrains such those of chalk and limestone ${ }^{32}$. Accordingly, the characteristic strontium compositions seen in some Scottish island communities have been linked to the use of kelp as food, fodder and fertilizer ${ }^{32}$. Seaweed, such as kelp, has high strontium content ${ }^{33-36}$ 
and thus abundant strontium with a seawater signature is released into the terrestrial biosphere and food chain when it is used to fertilize soils and as a food source ${ }^{35,36}$.

\section{Oxygen isotope data from Britain}

The best estimate of enamel phosphate oxygen isotope composition of individuals of British origin describes a near normal distribution (kurtosis $=0.3$, skewness $=-0.13$ ) with a mean of $17.7 \%$ o $\pm .7(1 \mathrm{SD}, \mathrm{n}=615)$ (Figure 4). This is in good agreement with the value of $17.7 \% \mathrm{~m} \pm$ 0.9 (2SD, $\mathrm{n}=55$ from 9 sites) proposed in Chenery et al. (2010). The distribution of the complete dataset is more asymmetric (kurtosis $=1.6$ and skewness $=-0.61$ ), with a mean of $17.6 \% \pm 0.8$ ( $1 \mathrm{SD}, \mathrm{n}=666)$. However, this includes data from exotic individuals from sites where there is evidence for non-British origin, comprising: 1) intrusive burials at the Lankhills cemetery in Winchester (Hampshire) classified as non-British on the basis of burial practice and burial goods ${ }^{8}$; 2) decapitated individuals from a Viking-Period execution pit near Weymouth (Dorset) interpreted as the executed crew of a long ship ${ }^{28}$ and 3) Roman Period burials of decapitated individuals from York ${ }^{13,17,38}$; 4) a Bronze Age individual with a rich burial assemblage including artefacts of continental origin ${ }^{39}$; 5) a Roman Period burial with eastern European artefacts ${ }^{40}$. We accept that there is a degree of circularity in this rejection procedure, but the exclusion of 51 samples represents a $7.1 \%$ rejection rate, and we feel that the resulting data are a more realistic representation of the British population.

The range of oxygen isotope values for local individuals within Britain is not unique ${ }^{41}$. As similar values can be found in France, northern Mediterranean countries ${ }^{42}$ and south eastern Norway, it is not possible to discriminate exotic (non-British) individuals from these locations using oxygen isotope values alone.

Information for the range of British $\delta^{18} \mathrm{O}_{\text {(VSMOW) }}$ in drinking water comes from two sources: 1) the contour map of Darling et al. ${ }^{46}$ representing geographic variation in recent spring and groundwater (dataset not available) and UK tap water values; 2) the British Geological Survey's (BGS) archive of shallow borehole and surface water data. The oxygen isotope map presents the weighted mean of spatially distributed $\delta^{18} \mathrm{O}_{\text {(VSMOW) }}$ values across Britain at $0.5 \%$ intervals ranging from $>-5.0 \%$ to $<8.5 \%$. We have calculated a mean for Britain of $-7.2 \%$, using the area distribution of this map, based on $1 \%$ contour intervals. While Darling et al. ${ }^{46}$ do not provide the whole dataset used to construct the map, they do provide data for tap water; these $\delta^{18} \mathrm{O}_{\text {(VSMOW) }}$ values range from -4.6\% (Lands End, extreme SW England) to 9.3\% (Cairngorms, Scotland). Although the range of water $\delta^{18} \mathrm{O}_{\text {(VSMOw) }}$ values for the 
Darling map range between $-4.0 \%$ and $-9.0 \%$, values above $-5.0 \%$ are spatially restricted and hence rare (see Table 1). The BGS data (Figure 5) provide a mean for Britain of -7.4\%o \pm 1.7 ( $n=975,2 \mathrm{SD}$ ) which is good agreement with our calculated. While the BGS dataset is not as comprehensive as the mapped data, they are sufficient for comparison with archaeological data in the discussion below. The equation 4 of Daux et al. ${ }^{3}$ was applied to our enamel data (excluding known exotic individuals), from which a mean of $-6.7 \%$ o \pm 1.2 (1SD, $\mathrm{n}=615$ ) was derived.

The mean and standard deviation for the British drinking water and for the calculated drinking water values derived from the archaeological tooth enamel do overlap, but they are not the same (Figure 6). This is largely caused by the inequalities in sample distribution across Britain (Figure 1), and the particular lack of archaeological enamel data from Scotland.

However, there are a number of other factors that might contribute to the imperfect match between actual and calculated water values that need to be taken into account when assigning a place of origin to an individual. These are: 1) the teeth that have been used include some that mineralized in early childhood when the consumption of breast milk was high; 2) ingested fluids were fractionated during food processing; 3 ) the climate has varied through time; 4) the dataset includes more exotic individuals from warmer than those from colder climates. We can address some of these issues, and in so doing, comment generally on the processes that may affect oxygen isotope results from human dental enamel.

\section{1) Choice of tooth}

The choice of tooth for analysis is important. Teeth mineralize at different times in childhood $^{43}$ and in order to relate the tooth enamel value to that of drinking water it is necessary to analyse teeth that do not have a pre-weaning dietary component. Breast milk oxygen is fractionated during the metabolic processes which take place in the body of the mother, such that enamel formed during breast feeding can be up to $2 \%$ higher in oxygen isotope composition than enamel formed after weaning. Although deciduous teeth are not included in this dataset, breastfeeding may have continued during the development of the permanent dentition that commences mineralization in the first year of life, particularly the incisors, canines and first molars. The oxygen isotope composition of first, second and third molars from thirty Anglo-Saxon individuals from the Lincoln area of Britain ${ }^{44}$ is presented in 
Figure 7a. These data show that M2 and M3 agree well with each other and that the earlier forming M1 enamel has a higher mean oxygen isotope value.

The main dataset has been acquired over many years and includes a mixture of tooth types and their range is displayed in Fig 7b. The data do not show the same relationship between the M1 and the M2 \& M3 as seen in the Lincolnshire dataset. This is probably because the relationship between the compositions of the teeth that form at different ages is masked by sources of greater variation such as that caused by the geographic distribution of archaeological sites. Hence, although the variation in tooth type may have contributed to the overall scatter of the data, it is not the primary cause of apparent offset between tooth enamel and British aquifer values.

\section{2) The effect of modified water sources}

Evaporation can shift the isotope compositions of potable water sources away from that of average regional rainwater, towards a higher range of oxygen isotope values. For example, lakes may show enrichment of 1-2\%o in temperate climates and much more in arid conditions ${ }^{45}$. This effect is recorded in the large lakes of northern Denmark where evaporation from the surface increases the $\delta^{18} \mathrm{O}$ value of the lake by 1.5 to $2 \%$ above rainwater ${ }^{46}$. This water then flows out of the lakes in rivers that transport enriched ${ }^{18} \mathrm{O}$ water across northern Denmark. The evaporative effect is minimal in Britain where meteoric ground water computations fall within $0.5 \%$ of average rainfall values ${ }^{47}$. Accordingly, for British born individuals it is unlikely that there is a significant shift caused by drinking from water sources modified by naturally occurring evaporative processes. However, drinking processed water (such as beer or tea), or deriving a significant proportion of water intake from cooked foods with a high water content such as stews, soups and porridges (i.e. pottage ) could result in a combined oxygen isotope value for water intake higher than that of the natural water composition of a local area. This effect is discussed in detail in Brettell et al. ${ }^{48}$.

\section{3) The effect of Climate change}

The last 10,000 years are well documented with respect to climate variation, however, there is still considerable uncertainty in the data and such uncertainties increase with increasing antiquity. It is estimated that a $1^{\circ} \mathrm{C}$ shift in the mean temperature is required to move the rainwater $\delta^{18} \mathrm{O}$ values by $0.57 \%{ }^{49}$, which equates to $c . \pm 0.37 \%$ in tooth enamel ${ }^{3}$. Such a temperature shift exceeds current estimates for changes in the British climate during the Holocene ${ }^{50}$. There are differences in the range of enamel $\delta^{18} \mathrm{O}$ values that we have 
documented through time (Figure 8). The data from the Neolithic Period is higher than other periods, which might be attributable to a warmer climate. However, most of the samples of Neolithic age are from sites on the west coast of Scotland, an area with the highest recorded groundwater oxygen isotope values in Britain. Climate effects can only be confirmed when isotope variations through time can be established from a restricted locality.

\section{4) Disproportionate numbers of unidentified exotic individuals.}

It is almost certain that the dataset includes individuals who did not spend their childhood in Britain. We have been very conservative in only rejecting 51 samples where there is evidence for non-British origin. However, there are likely to be other unidentified exotic individuals and if a greater proportion of these individuals originated within a climate zone with higher ambient drinking water values than lower (or vice versa) this will introduce a bias into the overall dataset.

The other factors that could influence the relationship between the water and enamel profiles are difficult to address on current evidence and remain as uncertainties in our interpretations. Well constrained studies are needed to assess these factors. In summary, the main causes of discrepancy between the water and enamel datasets is thought to arise from the different geographic sample distribution, the types of teeth used and the presence of unidentified immigrants.

\section{Subdividing oxygen isotope composition of British human enamel into two end member groups.}

Britain and Ireland have been contoured for variations in drinking water composition at the $0.5 \%$ and $1 \%$ interval, however, we do not yet have enough well provenanced archaeological data to test whether this level of contouring can be applied to direct measurements of human tooth enamel. It is, however, possible to identify within the existing British dataset into at least two biogeographic climate-groups that, to the best of our knowledge, record values from "local" individuals. This is, by nature, subjective but it should be possible to improve upon as more datasets become available.

The two chosen subsets of archaeological data represent bio-climatic areas of the British Isles which are also characterized by low rainfall, predominantly on the leeward (eastern) side of the country, and a higher rainfall zone that is largely restricted to the windward side of western and southern British Isles. The windward side records rainfall of $>1000 \mathrm{~mm} / \mathrm{A}$ ${ }^{51}$ and drinking water values that range between -4 to $-7 \%{ }^{47}$. Annual rainfall in the leeward 
side is $<1000 \mathrm{~mm}^{51}$ and drinking water ranges between -7 and $-9 \%{ }^{47}$ (see appendix). These two zones represent the extremes of a broad national trend in drinking water composition that is driven by long term patterns of atmospheric and oceanic circulation, and by a range of regional geographic variables ${ }^{47}$. For simplicity, we have named the selected subsets "windward" (where ambient $\delta^{18} \mathrm{O}_{\text {(VSMOw) }}$ values are likely to be higher) and "leeward"

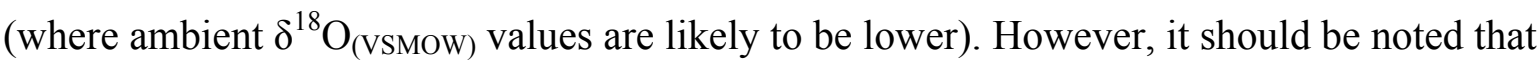
this is a simplification of the expected pattern of variation, and the geographic areas that they represent.

The archaeological subsets identified with the leeward area is comprised of the following datasets: two "local" Anglo Saxon populations from Rutland ${ }^{52-54}$; Auldhame, a Medieval village in Lothian ${ }^{16}$; the $18-19^{\text {th }}$ century AD populations for Chelsea where there is documentary evidence of the origin of most of the individuals ${ }^{10}$; three Bronze Age individuals from West Heslerton ${ }^{5}$. The windward subset corresponds to data from Scottish island populations defining "locals" by their high strontium concentrations and seawater isotope signature. These include individuals from Cnip, Galson ${ }^{7}$ South Uist, ${ }^{55}$ and Orkney ${ }^{56}$. England and Wales are represented by a Bronze Age individual from Great Orme ${ }^{57}$, two Anglo Saxon individuals from Anglesey and 25 Medieval individuals from Hereford Parish. The normal distribution curves and the $95 \%$ confidence intervals on the population means are given in Figure 9. The leeward area individuals have a mean of $17.2 \% \pm 1.3 \%$, $(2 \mathrm{SD}$, distribution skewness $=0.27$, kurtosis $=0.74, \mathrm{n}=83)$ this equates to a drinking water value of $-7.5 \%$ $\pm 1.8 \%$ o, $\left(2 \mathrm{SD}, \mathrm{n}=83^{3}\right)$ and the windward area individuals have a mean of $18.2 \%{ }_{0} \pm 1.0 \%,(2 \mathrm{SD}$, distribution skewness $=-0.18$, kurtosis $=0.22, \mathrm{n}=41)$. which equates to a drinking water value of $\left(-5.8 \%\right.$ o $\left.\pm 1.8 \%{ }^{3}\right)$. Both datasets conform to a normal distribution using the Anderson Darling normality test ( $\mathrm{p}=0.08$ and 0.35 respectively). An ANOVA analysis shows that the means of these two populations are significantly different from one another at $95 \% \mathrm{CI}$, supporting the hypothesis that these represent different populations. These two datasets provide a statistical definition for the oxygen isotope composition of human tooth enamel within two isotopically contrasting geographic zones within the British Isles.. It can be assumed that these statistical ranges represent the degree of ambient variation that might be seen within populations exposed to the extremes of drinking water composition within the British Isles. In the following sections the oxygen isotope data from a number of populations, sub-populations and individuals obtained from isotopically intermediate geographic zones are analysed on the basis of their relationship to these two control datasets. 


\section{Distinguishing between burial populations}

One of the primary aims of isotope studies is to identify individuals who are displaced relative to their childhood origin and, where possible, constrain their place of origin. Examples of this approach using oxygen isotope sub-populations are shown in Figure 10. The data from Catterick, a Romano-British garrison ${ }^{58}$, plots between the two defining populations in a central zone consistent with the geographic position of Catterick, which is broadly central with respect to the east and west coasts, and with the interpretation that most of the individuals were of local origin. ${ }^{58}$

The second example is a group of four Anglo-Saxon period individuals, excavated from a site (Easington) on the east coast on England, near Hull ${ }^{14}$. They should plot within data from the leeward area, if they are from the broader Easington area, but they overlap with the data from the more westerly windward area. This indicates that they were not raised on the east coast of England and highlights a feature of humans dating from the Anglo-Saxon period that has been observed before, namely that these populations tend to have higher $\delta^{18} \mathrm{O}_{\text {(VSMOW) }}$ values than would be expected from the region where they were buried ${ }^{22,59}$.

During the adventus Saxonum, migrants were documented as originating largely from the areas to the east and northeast of Britain, principally Denmark and Northern Germany, where rainfall $\delta^{18} \mathrm{O}_{\text {(VSMOW) }}$ values are the same or lower than those of Britain. Thus, higher values, which suggest a more westerly origin, are at odds with historically documented patterns of migration ${ }^{59}$. It has been suggested that the higher $\delta^{18} \mathrm{O}_{\text {(VSMOW) }}$ values may be a function of diet caused by a significant proportion of water coming from beer and potage, the production of which will enrich and hence elevate the oxygen isotope compositions relative to the source water supply ${ }^{48}$. However, the elevated $\delta^{18} \mathrm{O}_{\text {(VSMOW) }}$ values seen is some Anglo-Saxon groups may be a true indication of a non-local origin and be caused by drinking lake water ${ }^{60}$ enriched through evaporation as described above.

It is thus possible that the relatively high oxygen isotope ratios found in early Anglo-Saxon groups in the UK (such as those found at Easington) may be indicative of an origin in Jutland or other regions of Denmark, where lake water, and its run-off in rivers, is a major source of drinking water. Such a suggestion will need testing but it draws attention to the need to understand local conditions and behaviour when interpreting data.

\section{Subdividing data from a single burial site.}


Burials from cemeteries such as Lankhills, near Winchester ${ }^{8,61}$, display a wide range of oxygen isotope compositions. Some individuals have been proposed, on the basis of grave goods, to be of a continental origin ${ }^{62}$, and some, because of high, or extremely low ${ }^{87} \mathrm{Sr} /{ }^{86} \mathrm{Sr}$ values have been interpreted as of non-local origin; the remaining data form a distinct cluster. Using the drinking water conversion equation of Levinson, the conventional cut-off argument ${ }^{61}$ suggests that enamel phosphate oxygen isotope values above $18.6 \%$ lie outside the range for British drinking water and hence are of non-British origin. However, this interpretation depends upon the choice of drinking water equation used, and as shown by Pollard at al. ${ }^{63}$, introduces large regression errors into the debate.

The oxygen isotope composition of tooth enamel from individuals from Lankhills cemetery is shown in a probability density curve (Figure 11). The main peak centres on a value of $c$. $18.1 \%$, which equates to a drinking water value of $-5.9 \%$, using Daux et al $2010^{3}$ equation 4 which is close to the predicted values of $-6 \%$ to $-7 \%$ for that part of Britain ${ }^{47}$. There are a number of individuals with lower values, these are predominantly those identified as being of continental origin on the basis of grave goods ${ }^{8,62}$, and a group of 13 individuals, at the highest end of the range. If these 13 individuals are treated as a sub-group, and compared with the high rainfall area dataset, the sub-group are a population with an oxygen isotope composition that has a significantly higher mean at $95 \% \mathrm{CI}$ than the windward dataset.

Not only do the 13 individuals have a restricted and unusual range of oxygen isotope compositions, but they also show a restricted range of strontium isotope values: of $0.7089 \pm$ 0.0005 ( $1 \mathrm{SD}, \mathrm{n}=13)$. The strontium composition is typical of a limestone terrain and indistinguishable from the area in which they were found, namely on the Chalk. However, by combining the oxygen and strontium characteristics it is possible to assess the probability of their origin in Britain versus their origin from other likely places, in this case, elsewhere in the Roman Empire.

Niche modelling using both strontium and oxygen isotope data.

The combined probability of oxygen and strontium isotope values for Britain is given in Table 1 . This table is based on numerically combining the surface area expression of both the strontium biosphere values, derived from calculating the relative areas of isotope subdivision on the British strontium biosphere map ${ }^{30}$ and the area of drinking water isotope compositions derived from the $1 \%$ contoured map ${ }^{47}$. About $1 \%$ of the area of Britain can accommodate a combination of strontium isotope ratios below 0.7092 and drinking water values above $-6 \%$. 
The most likely place that could accommodate this combination is somewhere such as the Isle of Skye where oxygen isotope compositions are heaviest and strontium biosphere values might yield low enough ${ }^{87} \mathrm{Sr} /{ }^{86} \mathrm{Sr}$ values ${ }^{64}$.

This probability can be compared with data from the Roman Empire. This map was produced by calculating the area drinking water values, using modern rainwater data ${ }^{41}$, for the area of Europe occupied by the Roman Empire, excluding Britain. About 16\% of the Roman Empire outside Britain accommodates drinking water values between -3\%o and -5\% , mostly in the southern and eastern Mediterranean margins and eastern Spain (Figure 12). We do not have a map of the strontium biosphere values of the Empire but these can be estimated from the geology of the area. Mesozoic sediments, including limestone, are common in these areas ${ }^{65}$ with mineral waters recording values between $0.707-0.708^{66}$. Plant and human data from Syria $^{67}$, Greece ${ }^{68,69}$, Italy ${ }^{70}$ and Egypt ${ }^{71}$ also provide similar strontium isotope values. There are large areas of habitable land around the Mediterranean that can accommodate both the strontium and oxygen isotope values of the outlying individuals found in the Lankhills cemetery. Hence, the isotope combination can be quantified as $1 \%$ probability of a British origin (strontium and oxygen) against a 15\% probability (controlled by the drinking water distribution as limestone, with appropriate ${ }^{87} \mathrm{Sr} /{ }^{86} \mathrm{Sr}$ values, is widespread in this region ${ }^{65}$ ) of an origin within another part of the Roman Empire. This approach provides a probability of origin that, hopefully, is more informative than simply trying to demonstrate whether or not a group is of British origin.

When these methods are applied to an individual, rather than being able to compare data from populations, there is far more uncertainty, and claims that someone is or is not of a certain origin have hung on a combination of the drinking water equations used, the inter-laboratory compositions and reference material ${ }^{72-74}$. In the last section we discuss the interpretation of the data from a single individual using the example of the Amesbury Archer ${ }^{39}$.

\section{Characterizing the origin of an individual.}

The Amesbury Archer's grave is the richest Bronze Age burial in Britain ${ }^{39}$. He was excavated in 2002 along with an associated burial of a young man, referred to as the Archer's Companion. The two men had unusual non-metric traits in the bones of their feet suggesting they were biologically related ${ }^{39}$. The oxygen isotope composition of both teeth (P2 \& M3) from the Archer was 16.2\%o. In contrast, the companion shows a significant difference in $\delta^{18} \mathrm{O}_{\text {VSMow }}$ between two teeth with the earlier forming premolar having a value of $17.0 \%$ 
and a later forming $3^{\text {rd }}$ molar a value of $16.4 \%$. There has been much discussion of the origin of these individuals centring on whether the tooth enamel of the Archer excludes him from a British origin. This debate has hinged on calculating a drinking water value which, as shown by Pollard et $\mathrm{al}^{63}$, incurs large errors and depends upon the equation chosen.

The tooth enamel value of $16.2 \%$ is within the 3 sigma range of the British oxygen isotope values as defined above. In other words there is, at best, a $2.5 \%$ probability that he is of British origin based on oxygen. However, more than one tooth has been analysed. If the four tooth enamel analyses from the Archer and the Companion are treated as a population subset and compared with the leeward area dataset they are significantly different populations at 95\% confidence intervals (Figure 13).

Roman Period and earlier populations from Germany, Austria, Hungary and Czech Republic are dominated by ${ }^{87} \mathrm{Sr} /{ }^{86} \mathrm{Sr}$ values similar to that of the Archer's teeth ${ }^{75-77}$, and these parts of continental Europe also have drinking water/rainwater values which could supply the oxygen isotopes values recorded in his tooth enamel ${ }^{41,78}$.

So although isotope analysis cannot definitely provide an answer as to whether he spent his childhood in Britain it can provide evidence that the man in this unusually rich burial has an unusual oxygen isotope composition in his teeth that represents, at best, $5 \%$ of the British population and that there are areas in continental Europe where his combined strontium and oxygen isotope tooth composition would be far more typical of the parent population.

\section{Conclusions.}

The data compiled in this paper provide a reference dataset for British populations from the Neolithic to $18^{\text {th }}$ century AD.

The typical range of strontium isotope values is from 0.7078 to 0.7142 (England and Wales) and to 0.7165 (Scotland).

Strontium concentrations in Britain range between 20 and $180 \mathrm{ppm}$ but there is a trend towards high strontium concentrations associated with a ${ }^{87} \mathrm{Sr} /{ }^{86} \mathrm{Sr}$ ratio around 0.7092 . This feature appears to be related to the cultural practice of using seaweed to fertilise poor marginal soils.

Oxygen isotopes from tooth enamel in Britain display a normal distribution of data with a mean $17.7 \%$ o $\pm 0.7 \%$, $(1 \mathrm{SD}) \mathrm{n}=615$. Drinking water oxygen isotope values have a range of $7.41 \%$ $\pm 1.68 \%$ o $(\mathrm{n}=975,2 \mathrm{SD})$. 
Datasets based on "local" individuals define two subdivisions of Britain, based on human enamel values: leeward area $=17.2 \% 0 \pm 0.6 \%$, $(1 \mathrm{SD}, \mathrm{n}=83)$ and windward area $=18.2 \% \mathrm{t}$ $0.5 \%$ o $(1 \mathrm{SD}, \mathrm{n}=41)$.

These two populations can be used as control groups against which to compare populations of unknown origin. As this comparison is based on direct measurements of tooth enamel oxygen composition it removes the problem of having to convert to drinking water values with the associated uncertainties, choices and error expansion.

These data should provide a useful reference set for archaeological, forensic and environmental studies.

\section{Acknowledgments}

We thank the many collaborators who have allowed us to use and publish the data in this paper. We specifically thank, for access to the Hereford Cathedral skeletons, the BARC at the University of Bradford, Anthea Boylston, Darlene Weston, Alan Ogden and Derek Hurst. Thanks to Geraldine Wildman for producing the map of oxygen isotope variation across the Roman Empire, Joseph Warham for his constructive comments and Chris Milne for extracting the drinking water data from the BGS database.

\section{References}

1. R. A. Bentley, Journal of Archaeological Method and Theory, 2006, 13, 135-187.

2. A. A. Levinson, B. Luz and Y. Kolodny, Appl. Geochem., 1987, 2, 367-371.

3. V. Daux, C. Lecuyer, M. A. Heran, R. Amiot, L. Simon, F. Fourel, F. Martineau, N. Lynnerup, H. Reychler and G. Escarguel, J. Hum. Evol., 2008, 55, 1138-1147. DOI: 10.1016/j.jhevol.2008.06.006.

4. C. Chenery, G. Müldner, J. Evans, H. Eckardt and M. Lewis, J. Arch Sci., 2010, 37, 150-163. DOI: 10.1016/j.jas.2009.09.025.

5. J. Montgomery, University of Bradford, 2002.

6. J. Montgomery, P. Budd and J.A.Evans, European Journal of Archaeology, 2000, 3, 407-422.

7. J. Montgomery, J. A. Evans and T. Neighbour, J Geol Soc, 2003, 160, 649-653.

8. J. Evans, N. Stoodley and C. Chenery, J. Arch Sci., 2006, 33, 265-272.

9. J. A. Evans, C. A. Chenery and A. P. Fitzpatrick, Archa, 2006, 48, 309-321.

10. M. A. Trickett, Durham University, 2007.

11. H. Sale, University of Bradford, 2008.

12. K. Hemer, Proceedings of the Isle of Man Natural History and Antiquarian Society 2012, Volume XII

13. G. Müldner, C. Chenery and H. Eckardt, Journal of Archaeological Science, 2011, 38, 280 290. DOI: $10.1016 /$ j.jas.2010.09.003.

14. J. Richardson, Diane Alldritt, Craig Barclay, I. Brooks, J. Carrott, C. Chernery, H. Cool, J. Cowgill, P. Didsbury, J. Evans, C. Fern, G. Gaunt, K. Hartley, D. Heslop, M. Holst, T. 
Manby, E. Morris, F. Wild and D. Williams, Yorkshire Archaeological Journal, 2011, 83, 59-100.

15. S. Hughes, C. Chenery, J. Evans, A. Millard, S. Lucy, G. Nowell and G. Pearson, in prep.

16. E. Hindmarch, ed., Living and dying at Auldhame, ast Lothian: the excavation of an Anglican monastic settlement and Medieval parish church. in press.

17. J. Montgomery, Knüsel, C., and Tucker, K.,, ed., Identifying the origins of decapitated male skeletons from 3 Driffield Terrace, York, through isotope analysis: reflections of the cosmopolitan nature of Roman York in the time of Caracalla, University Press of Florida, Gainesville. 2011.

18. J. Montgomery, J. A. Evans and C. A. Chenery, in Wasperton: A Roman, British and AngloSaxon Cemetery in Central England. Woodbridge:, ed. M. O. H. Carver, C. Hills and J. Scheschkewitz, Boydell and Brewer. 2009, pp. 46-47.

19. S. Leach, H. Eckardt, C. Chenery, G. Mueldner and M. Lewis, Antiquity, 2010, 84, 131-145.

20. A. L. Lamb, M. Melikian, R. Ives and J. A. Evans, J. Anal. At. Spectrom., 2012.

21. E. Biddulph and K. Welsh, Cirencester before Corinium: excavations at Kingshill North, Cirencester, Gloucestershire, , 2011.

22. P. Budd, C. Chenery, J. Montgomery, J. Evans and D. Powlesland, Plasma Source Mass Spectrometry: Applications and Emerging Technologies, 2003, 195-208.

23. E. Biddulph, K. Walsh, major_contibution_by, D. Mullen, other_contributions_by, L. Allen, P. Booth, C. Champness, S. Clough, J. Cotter, JaneEvans, R. Ixar, L. Keys, A. Lamb, R. Nicholson, C. Poole, F. Roe, I. Scott, L. Strid, R. Shaffey, W. Smith, d. Stansbie, R. Taylor, J. Timby, H. Webb and A. Zochowski, in Thames Valley Landscapes Oxford Archaeology. 2011, vol. 34.

24. R. A. Hall, J. Buckberry, R. Storm, P. Budd, W. D. Hamilton and G. McCormac, Yorkshire Archaeological Journal, 2008, 80, 55-92.

25. C. A. Chenery and J. A. Evans, Yorkshire Archaeological Journal, in press.

26. C. Trueman, C. Chenery, D. A. Eberth and B. Spiro, J Geol Soc, 2003, 160, 895-901. DOI: 10.1144/0016-764903-019.

27. N. Woodcock and R. Strachan, eds., Geological History of Britain and Ireland, Blackwell Science, Oxford. 2000.

28. C. Chenery, J.A.Evans, D. Score and A. Boyle, Journal of North Atlantic submitted.

29. J. A. Evans, J. Montgomery, G. Wildman and N. Boulton, J Geol Soc, 2010, 167, 1-4. DOI: 10.1144/0016-76492009-090.

30. J. A. Evans, J. Montgomery, G. Wildman and N. Boulton, Journal of the Geological Society, 2010, 167, 1-4. DOI: 10.1144/0016-76492009-090.

31. J. D. Blum, E. H. Taliaferro and R. T. Holmes, Oecologia, 2001, 126, 569-574.

32. J. Montgomery, J. A. Evans and R. E. Cooper, Appl. Geochem., 2007, 22, 1502-1514.

33. V. Romari's-Hortas, C. Garcia-Sartal, M. C. Barciela-Alonso, A. Moreda-Pineiro and P. Bermejo-Barreta, Journal of Agricultural and Food Chemistry, 2010, 58, 1986-1992.

34. D. Dungworth, P. Degryse and J. Schneider, in Studies in Archaeological Sciences - Isotopes in Vitreous Material., ed. P. Degryse, J. Henderson and G. Hodgkin, Leuven University Press., Leuven. 2009.

35. Y. A. Sapozhnikov, S. N. Kalmykov, I. P. Efimov and V. P. Remez, Appl. Radiat. Isot., 1996, 47, 887-888.

36. T. Morita, K. Fujimoto, H. Kasai, H. Yamada and K. Nishiuchi, J. Environ. Monit., 2010, 12, 1179-1186. DOI: 10.1039/b920173d.

37. J. Montgomery, Evans, J.A., and Chenery, C.A., ed., Combined lead, strontium and oxygen isotope analysis of the female adult from High Pasture Cave, Isle of Skye, Historic Scotland, Edinburgh. 2007. 
38. J. Montgomery, C. Knüsel and K. Tucker, in The Bioarchaeology of the Human Head: Decapitation, Decoration and Deformation, , ed. M. Bonogofsky, University Press of Florida, Gainesville. 2011, pp. 141-178.

39. A. P. Fitzpatrick, Excavations at Boscombe Down, Wessex Archaeology, 2011.

40. C. Heighway, Review of the Gloucester and District Archaeological Research Society, in press.

41. IAEA/WMO, in The GNIP database. 2006.

42. S. P. Lykoudis and A. A. Argiriou, Journal of Geophysical Research-Atmospheres, 2007, 112. DOI: $10.1029 / 2007 j \mathrm{~d} 008472$.

43. S. Hillson, Dental Anthropology, Cambridge University Press, Cambridge. 1996.

44. P. Macpherson, Sheffield, 2006.

45. W. G. Darling, A. H. Bath, J. J. Gibson and K. .Rozanski, in Isotopes in Palaeoenvironmental Reseach, ed. M.J.Leng, Springer, Netherlands. 2006, vol. 10, ch. 1, pp. 1-52.

46. T. Fronval, N. B. Jensen and B. Buchardt, Geology, 1995, 23, 463-466.

47. W. C. Darling, A. H. Bath and J. C. Talbot, HESS, 2003, 7, 183-195.

48. R. Brettell, J. Montgomery and J. Evans, Journal of Analytical Atomic Spectroscopy., in press.

49. W. Dansgaard, Tell, 1964, 16, 171-177.

50. R. A. Rohde. 2005.

51. M. O. UK.

52. S. Tatham, Leicester University, 2004.

53. J. A. Evans, S. Tatham, S. R. Chenery and C. A. Chenery, Appl. Geochem., 2007, 22, 19942005.

54. J. A. Evans and S. Tatham, in Forensic Geoscience: Principles, Techniques and Applications., ed. K.Pye and D.J.Croft, Geological Society, London, Bath. 2004, vol. 232, pp. 237-248.

55. M. P. Pearson, A. Chamberlain, O. Craig, P. Marshall, J. Mulville, H. Smith, C. Chenery, M. Collins, G. Cook, G. Craig, J. Evans, J. Hiller, J. Montgomery, J. L. Schwenninger, G. Taylor and T. Wess, Antiquity, 2005, 79, 529-546.

56. R. Toolis, w. contributions, J. Barrett, N. Boulton, C. Chenery, J. Evans, D. Hall, A. MacSween, M. Melikian and M. Richards, Proceedings Society Antiquaries of Scotland, 2008, 138, 239-266.

57. J. A. Evans and C. A. Chenery, Strontium and oxygen analysis of tooth enamel from sample P- P16379 from Great Orme, Llandudno, Wales, 2005.

58. C. Chenery, H. Eckardt and G. Müldner, J. Arch Sci., 2011, 38, 1525-1536. DOI: 10.1016/j.jas.2011.02.018.

59. R. Brettell, S. Marzinzik, J. Evans and J. Montgomery, European Journal of Archaeology, in press.

60. K. M. Frei and R. Frei, Applied Geochemistry, 2011, 26, 325-340. DOI: 10.1016/j.apgeochem.2010.12.006.

61. H. Eckardt, C. Chenery, P. Booth, J. A. Evans, A. Lamb and G. Muldner, J. Arch Sci., 2009, 36, 2816-2825. DOI: 10.1016/j.jas.2009.09.010.

62. G. Clarke, Pre-Roman and Roman Winchester. Part II: the Roman cemetery at Lankhills. , University of Oxford, Oxford. 1979.

63. A. M. Pollard, M. Pellegrini and J. A. Lee-Thorpe, Americal Journal of Physical Anthropology, 2011.

64. J. A. Evans, J. Montgomery and G. Wildman, J Geol Soc, 2009, 166, 617-631. DOI: 10.1144/0016-76492008-043.

65. K. Asch. 2005. 
66. S. Voerkelius, G. D. Lorenz, S. Rummel, C. R. Quétel, G. Heiss, M. Baxter, B.-P. C. , P. Deters-Itzelsberger, S. Hoelzl, J. Hoogewerff, E. Ponzevera, M. V. Bocxstaele and H. Ueckermann, Food Chem., 2010, 118, 933-940.

67. J. Henderson, J. Evans and Y. Barkoudah, Antiquity, 2009, 83, 414-429.

68. A. Nafplioti, in The prehistory of the island of Kythnos,( Cyclades, Greece) and the Mesolithic settlement at Maroulas, ed. A. Sampson, M. Kaczanowska and J. K. Kozlowski, The Polish Academy of Arts and Sciences. 2010, pp. 207-215.

69. A. Nafplioti, J. Arch Sci., 2008, 35, 2307-2317. DOI: 10.1016/j.jas.2008.03.006.

70. K. Killgrove, J. Montgomery and R. Tykot, Am. J. Phys. Anthropol., 2011, 144, 185-185.

71. J. Henderson, J. Evans and K. Nikita, Mediterranean Archaeology \& Archaeometry, 2010, 10, $1-24$.

72. L. S. Bell, Lee Thorp, J. A., and Elkerton, A, J. Arch Sci., 2009, 36, 166-173.

73. L. S. Bell, Lee-Thorp, J. A., and Elkerton, A.,, J. Arch Sci., 2010, 37, 683-686.

74. A. R. Millard and H. Schroeder, J. Arch Sci., 2010, 37, 680-682.

75. G. Grupe, T. D. Price, P. Schroter, F. Sollner, C. M. Johnson and B. L. Beard, Appl. Geochem., 1997, 12, 517-525.

76. T. D. Price, J. Wahl and R. A. Bentley, European Journal of Archaeology, 2006, 9, 259-284.

77. M. M. Schweissing and G. Grupe, J. Arch Sci., 2003, 30, 1373-1383.

78. K. Rozanski, in Problems of stable isotopes in tree rings, lake sediments and peat bogs as climatic evidence for the Holocene ed. B. Frenzel, B. Stauffer and M. Weiß, Fischer, Stuttgart 1995, vol. 15, pp. 171-186. 


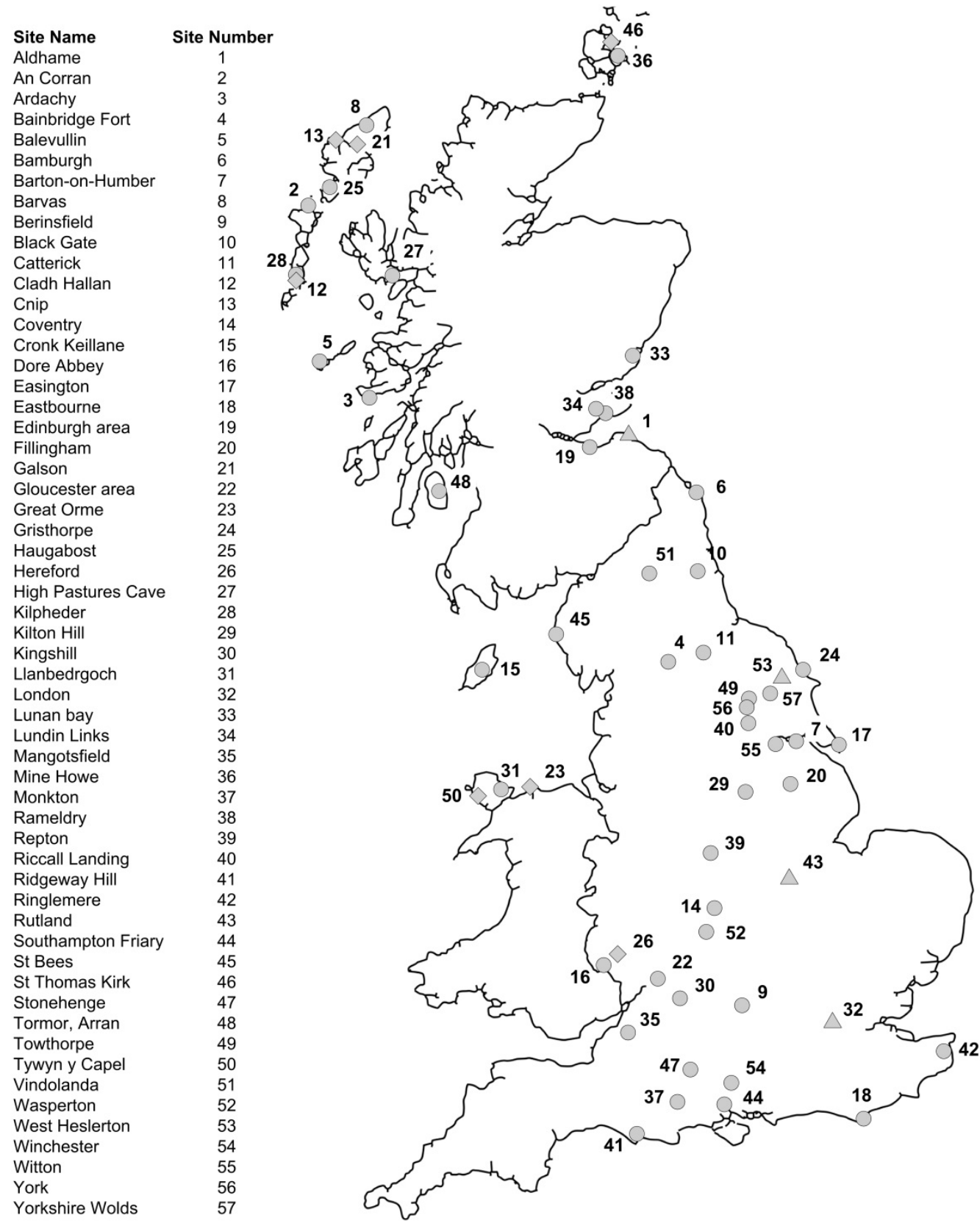



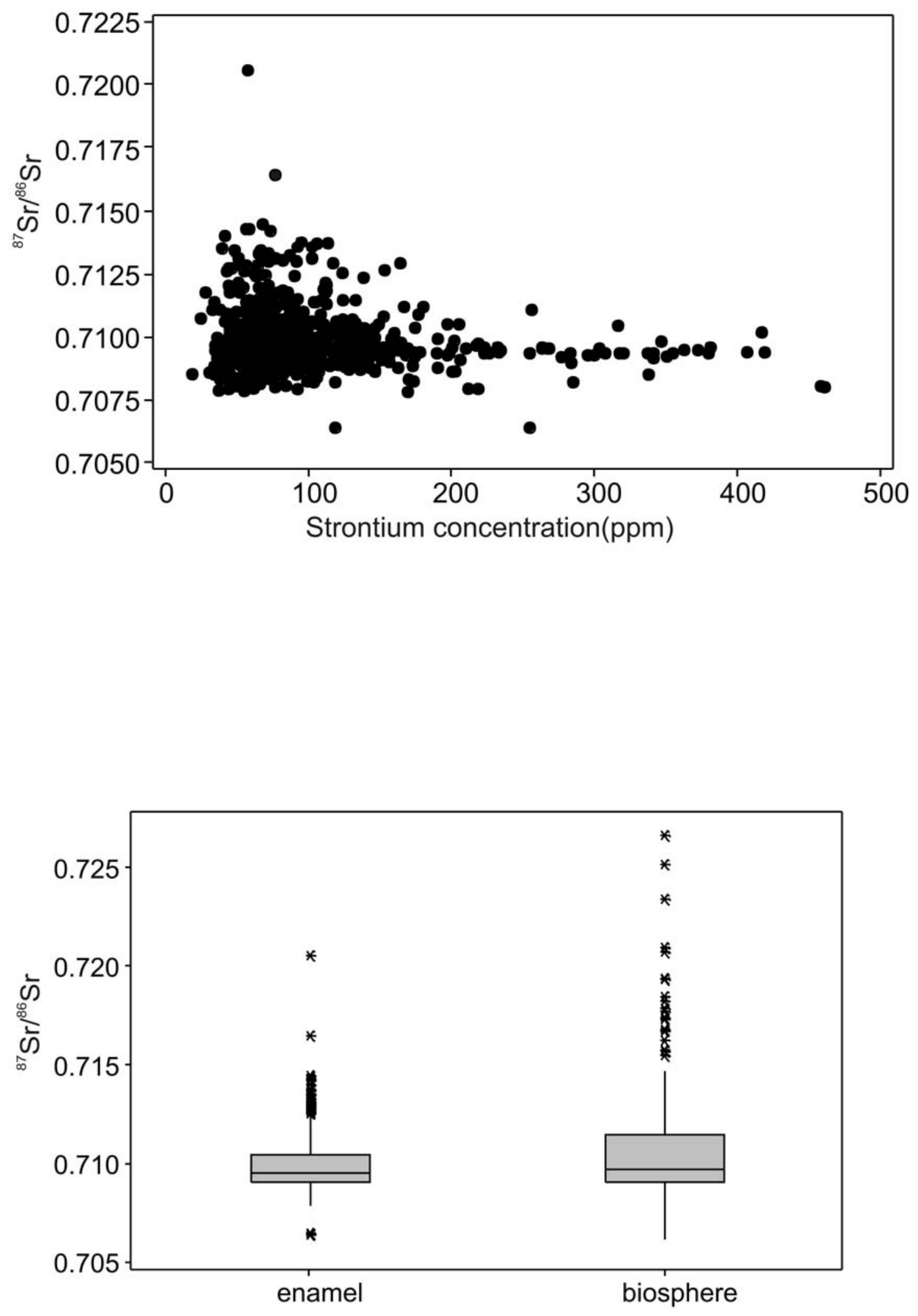


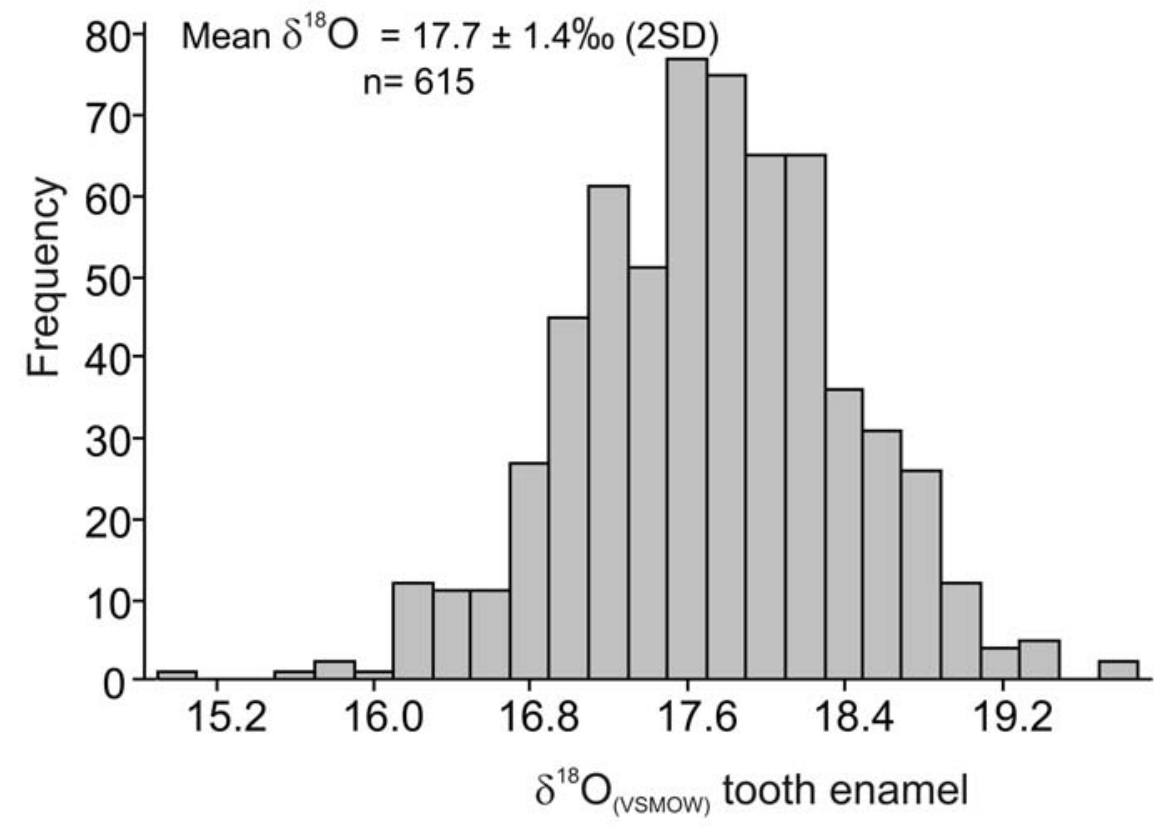




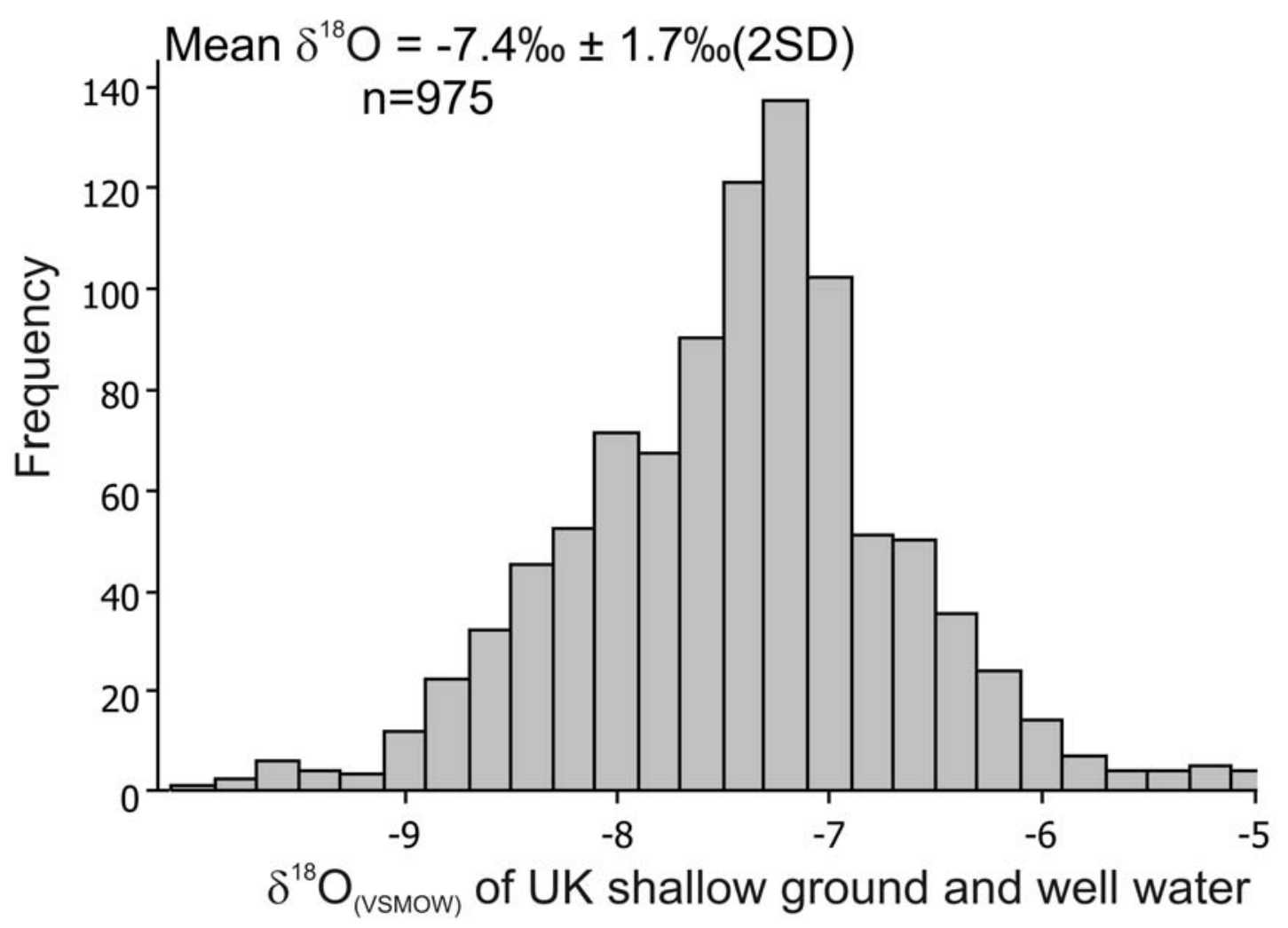



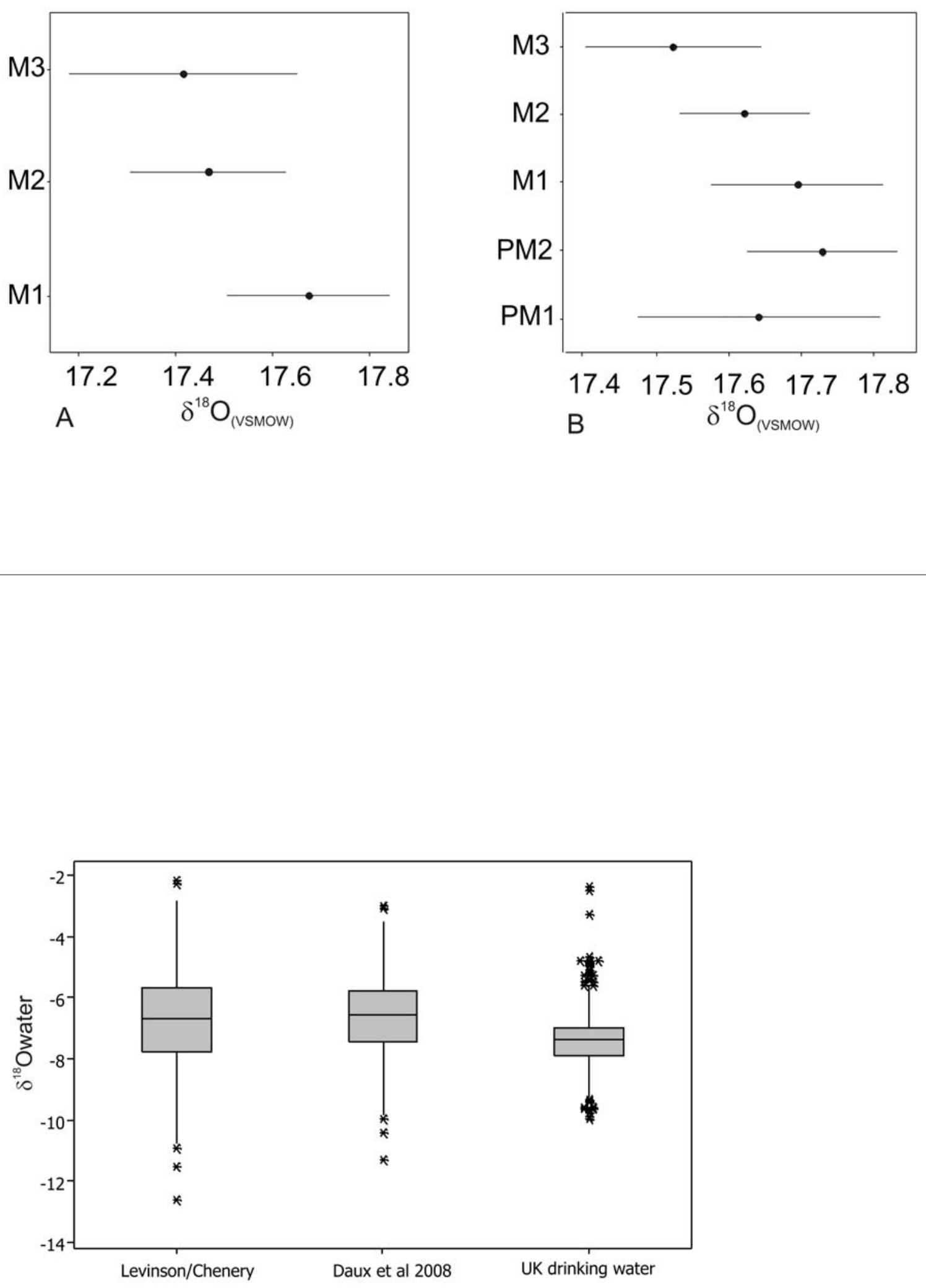


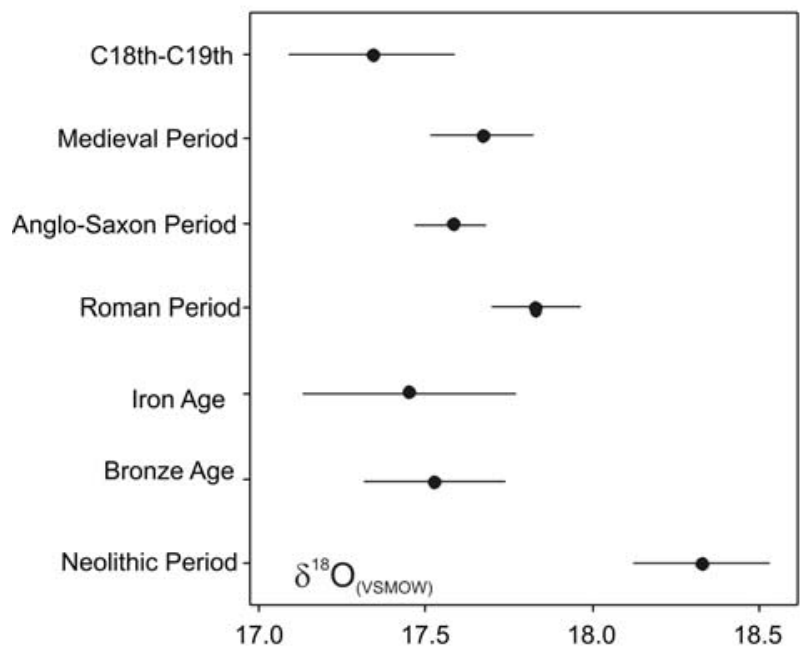


$95 \%$ confidence intervals on the population means
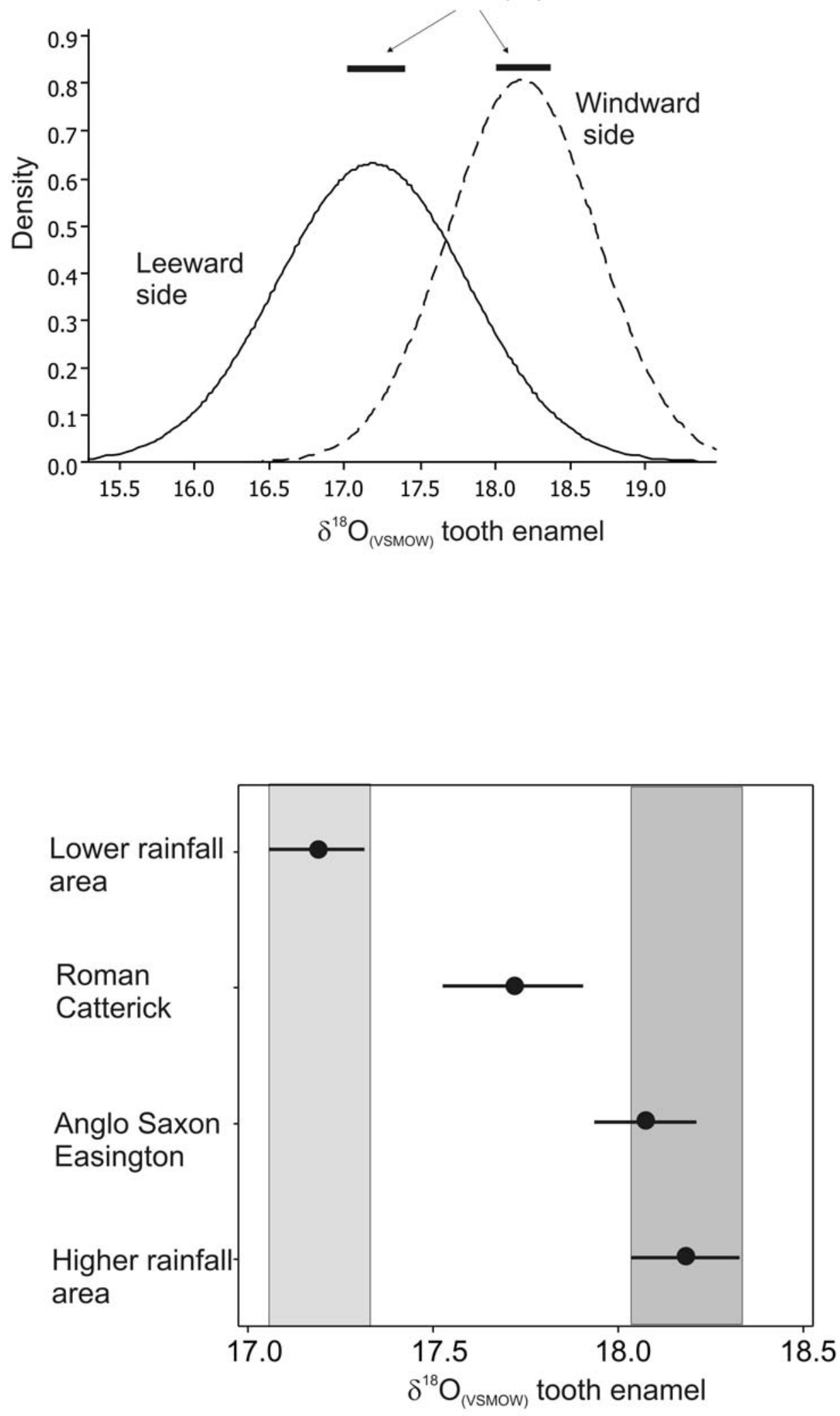


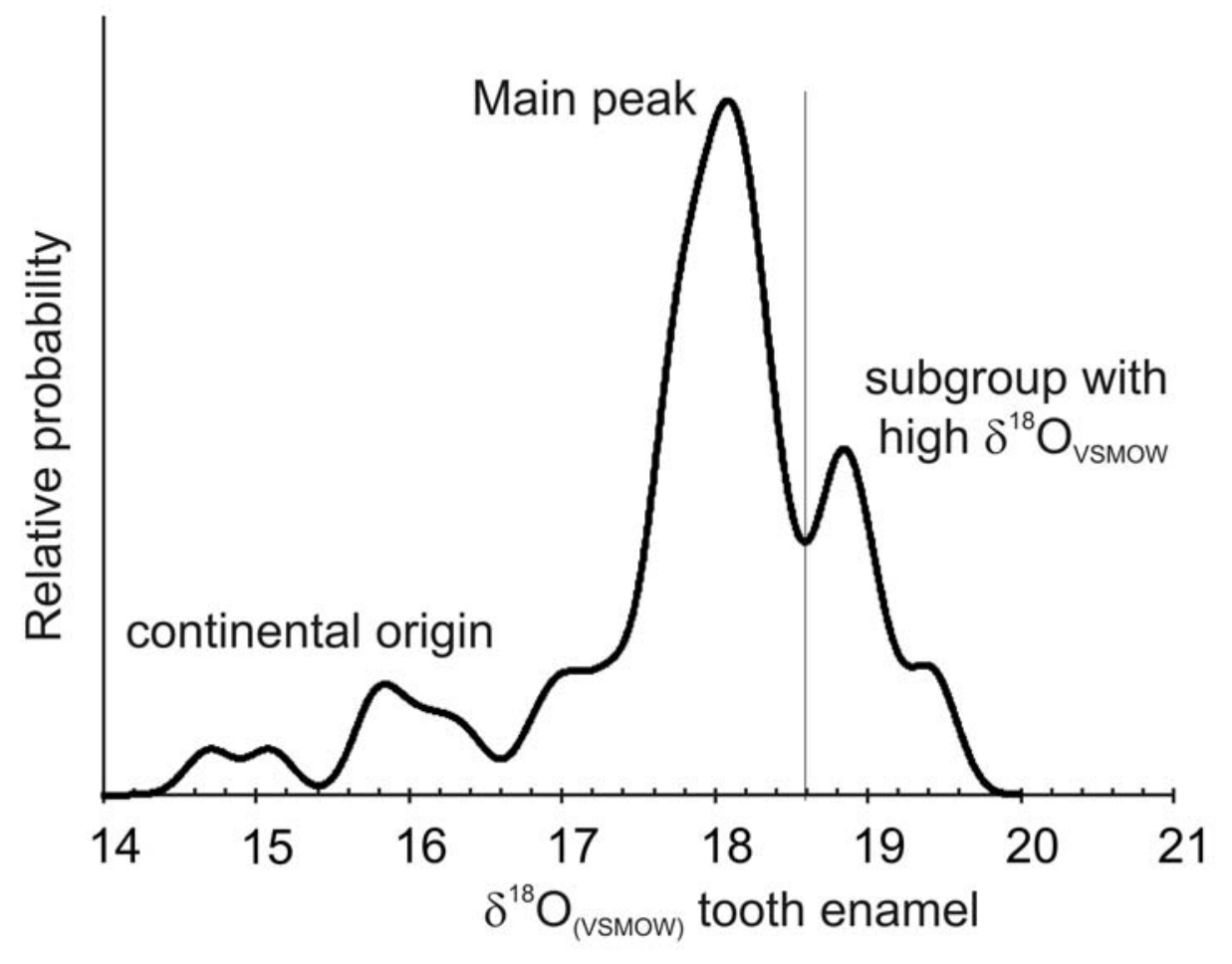



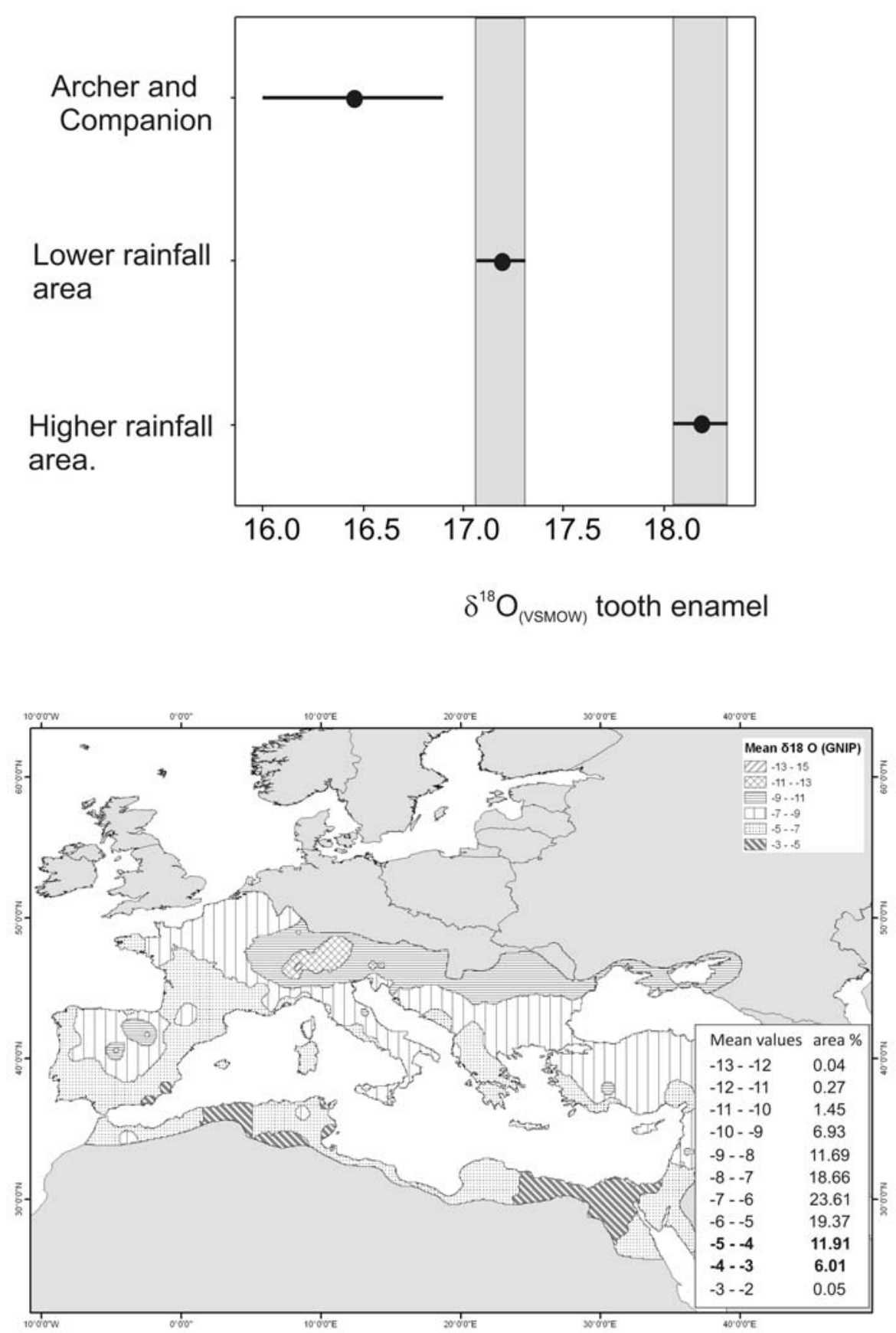


\begin{tabular}{|c|c|c|c|c|c|c|c|}
\hline Sample & Tooth & $\mathrm{Sr}(\mathrm{mg} / \mathrm{kg})$ & ${ }^{87} \mathrm{Sr} /{ }^{86} \mathrm{Sr}$ & $\delta^{18} 0($ VSMOW $)$ & Age & Site & $\begin{array}{c}\text { higher /lower } \\
\text { rainfall }\end{array}$ \\
\hline AL18 & M2 & 69 & 0.710981 & & Bronze Age & Aldro & \\
\hline AL19 & $\mathrm{C}$ & 39 & 0.708315 & 18.4 & Bronze Age & Aldro & \\
\hline AL20 & pM2 & 66 & 0.709818 & 17.7 & Bronze Age & Aldro & \\
\hline $\mathrm{Al} 21$ & $\mathrm{pM} 1$ & 35 & 0.708512 & & Bronze Age & Aldro & \\
\hline $\mathrm{Al} 22$ & M1 & 31 & 0.708588 & 18.3 & Bronze Age & Aldro & \\
\hline AL23 & $\mathrm{pM} 2$ & 40 & 0.708361 & 18.6 & Bronze Age & Aldro & \\
\hline Bore-2 & M2 & 149 & 0.709292 & & Iron Age & An Corren & \\
\hline Bore-3 & M2 & 107 & 0.709512 & & Iron Age & An Corren & \\
\hline AULD-SK-071 & M2 & & & 17.4 & Medieval & Auldhame & \\
\hline AULD-SK-074 & M2 & 158 & 0.709926 & 18.1 & Medieval & Auldhame & Lower rainfall \\
\hline AULD-SK-122 & M2 & 143 & 0.708751 & 17.1 & Medieval & Auldhame & Lower rainfall \\
\hline AULD-SK-158 & M2 & 92 & 0.713542 & 17.3 & Medieval & Auldhame & Lower rainfall \\
\hline AULD-SK-216 & M2 & 75 & 0.709339 & 18.2 & Medieval & Auldhame & Lower rainfall \\
\hline AULD-SK-219 & M2 & 84 & 0.710213 & 17.2 & Medieval & Auldhame & Lower rainfall \\
\hline AULD-SK-318 & M2 & 85 & 0.709637 & 16.5 & Medieval & Auldhame & Lower rainfall \\
\hline AULD-SK-327 & M2 & 142 & 0.709484 & 19.0 & Medieval & Auldhame & Lower rainfall \\
\hline AULD-SK-352 & M2 & 92 & 0.708903 & 16.5 & Medieval & Auldhame & Lower rainfall \\
\hline AULD-SK-394 & M2 & 140 & 0.709329 & 17.3 & Medieval & Auldhame & Lower rainfall \\
\hline AULD-SK-467 & M2 & 178 & 0.709390 & 16.9 & Medieval & Auldhame & Lower rainfall \\
\hline AULD-SK-520 & M2 & 99 & 0.710593 & 16.9 & Medieval & Auldhame & Lower rainfall \\
\hline AULD-SK-626 & M2 & 200 & 0.708647 & 18.0 & Medieval & Auldhame & Lower rainfall \\
\hline AULD-SK-663 & M2 & 460 & 0.708012 & 17.4 & Medieval & Auldhame & Lower rainfall \\
\hline AULD-SK-669 & M2 & 285 & 0.708189 & 17.6 & Medieval & Auldhame & Lower rainfall \\
\hline AULD-SK-714 & M2 & 128 & 0.710306 & 18.3 & Medieval & Auldhame & Lower rainfall \\
\hline AULD-SK-733 & M2 & 108 & 0.710841 & 16.8 & Medieval & Auldhame & Lower rainfall \\
\hline AULD-SK-742 & $\mathrm{M} 2$ & 137 & 0.710037 & 17.7 & Medieval & Auldhame & Lower rainfall \\
\hline AULD-SK-752 & M2 & 168 & 0.709236 & 17.5 & Medieval & Auldhame & Lower rainfall \\
\hline AULD-SK-868 & M2 & 167 & 0.711162 & 17.5 & Medieval & Auldhame & Lower rainfall \\
\hline AULD-SK-883 & M2 & 203 & 0.708647 & 18.2 & Medieval & Auldhame & Lower rainfall \\
\hline SF 614 & M1 & 124 & 0.712550 & 18.6 & Roman & Bainbridge Fort & \\
\hline SF 615 & M2 & 83 & 0.709940 & 17.9 & Roman & Bainbridge Fort & \\
\hline Tire-20 & M2 & 355 & 0.709344 & & Neolithic & Balevullin & \\
\hline B99 135 & no record & 33 & 0.711035 & 17.2 & Medieval & Bamburgh & \\
\hline BAM $99-130$ & no record & 78 & 0.711197 & 17.4 & Medieval & Bamburgh & \\
\hline BAM 99-129 & no record & 47 & 0.710937 & 18.0 & Medieval & Bamburgh & \\
\hline BH 99 124a & no record & 28 & 0.711740 & 17.8 & Medieval & Bamburgh & \\
\hline $\mathrm{BH} 99$ 124b & no record & 25 & 0.710747 & 18.6 & Medieval & Bamburgh & \\
\hline BH 99-134 & no record & 55 & 0.710478 & 16.0 & Medieval & Bamburgh & \\
\hline BH 99-135 & no record & & & 17.2 & Medieval & Bamburgh & \\
\hline $\mathrm{BH} 98$ & no record & 152 & 0.710772 & & Medieval & Bamburgh & \\
\hline D48 & $\mathrm{pM} 2$ & 65 & 0.711486 & 15.0 & Medieval & Bamburgh & \\
\hline D49 & $\mathrm{pM} 2$ & 80 & 0.710649 & 17.1 & Medieval & Bamburgh & \\
\hline D50 & $\mathrm{pM} 2$ & 38 & 0.711052 & 17.2 & Medieval & Bamburgh & \\
\hline D51 & $\mathrm{pM} 2$ & 132 & 0.710052 & 17.7 & Medieval & Bamburgh & \\
\hline D52 & M2 & 59 & 0.708925 & 17.6 & Medieval & Bamburgh & \\
\hline D53 & M3 & 62 & 0.711084 & 16.8 & Medieval & Bamburgh & \\
\hline D54 & $\mathrm{pM} 2$ & 68 & 0.710946 & 16.2 & Medieval & Bamburgh & \\
\hline D55 & M2 & 116 & 0.709429 & 17.1 & Medieval & Bamburgh & \\
\hline D56 & $\mathrm{pM} 2$ & 137 & 0.709830 & 16.8 & Medieval & Bamburgh & \\
\hline D57 & M2 & 65 & 0.709816 & 17.6 & Medieval & Bamburgh & \\
\hline D58 & $\mathrm{pM} 2$ & 78 & 0.710510 & 17.0 & Medieval & Bamburgh & \\
\hline D59 & M3 & 115 & 0.709957 & 16.6 & Medieval & Bamburgh & \\
\hline
\end{tabular}




\begin{tabular}{|c|c|c|c|c|c|c|c|}
\hline D60 & pM2 & 125 & 0.710162 & 17.2 & Medieval & Bamburgh & \\
\hline BH0580-M1 & M1 & & & 18.8 & Anglo-Saxon & Barton upon Humber & \\
\hline BH1105-M1 & M1 & & & 17.1 & Anglo-Saxon & Barton upon Humber & \\
\hline BH1105-M2 & M2 & & & 17.0 & Anglo-Saxon & Barton upon Humber & \\
\hline BH1105-M3 & M3 & & & 17.3 & Anglo-Saxon & Barton upon Humber & \\
\hline BH1136-M1 & M1 & & & 17.1 & Anglo-Saxon & Barton upon Humber & \\
\hline BH1136-M2 & M2 & & & 16.9 & Anglo-Saxon & Barton upon Humber & \\
\hline BH1136-M3 & M3 & & & 17.5 & Anglo-Saxon & Barton upon Humber & \\
\hline BH1327-M2 & M2 & & & 17.2 & Anglo-Saxon & Barton upon Humber & \\
\hline BARV-11 & M2 & 156 & 0.709249 & 18.5 & Iron Age & Barvas & Higher rainfall \\
\hline Ber-001 & pM2 & 86 & 0.709245 & 17.8 & Anglo-Saxon & Berinsfield & \\
\hline Ber-004 & pM2 & 91 & 0.711151 & 17.5 & Anglo-Saxon & Berinsfield & \\
\hline Ber-005 & M2 & 57 & 0.709493 & 18.3 & Anglo-Saxon & Berinsfield & \\
\hline Ber-006 & pM2 & 55 & 0.709795 & 17.1 & Anglo-Saxon & Berinsfield & \\
\hline Ber-008 & pM2 & 68 & 0.709128 & 18.1 & Anglo-Saxon & Berinsfield & \\
\hline Ber-010 & pM2 & 47 & 0.709157 & 17.6 & Anglo-Saxon & Berinsfield & \\
\hline Ber-018 & pM2 & 75 & 0.709258 & 17.7 & Anglo-Saxon & Berinsfield & \\
\hline Ber-020 & pM2 & 57 & 0.708959 & 18.0 & Anglo-Saxon & Berinsfield & \\
\hline Ber-026 & M2 & 79 & 0.709339 & 18.2 & Anglo-Saxon & Berinsfield & \\
\hline Ber-030 & pM2 & 79 & 0.708820 & 17.6 & Anglo-Saxon & Berinsfield & \\
\hline Ber-042 & pM2 & 76 & 0.708328 & 18.2 & Anglo-Saxon & Berinsfield & \\
\hline Ber-049 & M3 L & 93 & 0.709066 & 18.1 & Anglo-Saxon & Berinsfield & \\
\hline Ber-054 & M2 & 49 & 0.709427 & 18.3 & Anglo-Saxon & Berinsfield & \\
\hline Ber-061 & pM2 & 65 & 0.709302 & 17.6 & Anglo-Saxon & Berinsfield & \\
\hline Ber-073 & pM2 & 37 & 0.709433 & 17.6 & Anglo-Saxon & Berinsfield & \\
\hline Ber-081 & M2 & 60 & 0.708364 & 17.3 & Anglo-Saxon & Berinsfield & \\
\hline Ber-141 & pM2 & 46 & 0.709321 & 17.5 & Anglo-Saxon & Berinsfield & \\
\hline Ber-150 & pM2 & 65 & 0.709732 & 17.8 & Anglo-Saxon & Berinsfield & \\
\hline Ber-152 & pM2 & 77 & 0.709463 & 18.1 & Anglo-Saxon & Berinsfield & \\
\hline BG030-M1 & M1 & & & 17.8 & Anglo-Saxon & Black Gate Newcastle & \\
\hline BG030-M2 & M2 & & & 17.7 & Anglo-Saxon & Black Gate Newcastle & \\
\hline BG030-M3 & M3 & & & 17.9 & Anglo-Saxon & Black Gate Newcastle & \\
\hline BG040-M1 & M1 & & & 17.9 & Anglo-Saxon & Black Gate Newcastle & \\
\hline BG040-M2 & M2 & & & 17.8 & Anglo-Saxon & Black Gate Newcastle & \\
\hline BG040-M3 & M3 & & & 18.0 & Anglo-Saxon & Black Gate Newcastle & \\
\hline BG048-M1 & M1 & & & 17.7 & Anglo-Saxon & Black Gate Newcastle & \\
\hline BG248-M1 & M1 & & & 17.7 & Anglo-Saxon & Black Gate Newcastle & \\
\hline BG248-M2 & M2 & & & 17.4 & Anglo-Saxon & Black Gate Newcastle & \\
\hline BG252-M1 & M1 & & & 18.3 & Anglo-Saxon & Black Gate Newcastle & \\
\hline BG252-M2 & M2 & & & 17.4 & Anglo-Saxon & Black Gate Newcastle & \\
\hline BG252-M3 & M3 & & & 17.5 & Anglo-Saxon & Black Gate Newcastle & \\
\hline BG314-M1 & M1 & & & 17.9 & Anglo-Saxon & Black Gate Newcastle & \\
\hline BG314-M2 & M2 & & & 17.8 & Anglo-Saxon & Black Gate Newcastle & \\
\hline BG314-M3 & M3 & & & 17.3 & Anglo-Saxon & Black Gate Newcastle & \\
\hline BG365-M1 & M1 & & & 17.9 & Anglo-Saxon & Black Gate Newcastle & \\
\hline BG386-M1 & M1 & & & 17.6 & Anglo-Saxon & Black Gate Newcastle & \\
\hline BG386-M2 & M2 & & & 17.4 & Anglo-Saxon & Black Gate Newcastle & \\
\hline BG386-M3 & M3 & & & 17.5 & Anglo-Saxon & Black Gate Newcastle & \\
\hline BG422-M1 & M1 & & & 17.2 & Anglo-Saxon & Black Gate Newcastle & \\
\hline BG433-M1 & M1 & & & 17.1 & Anglo-Saxon & Black Gate Newcastle & \\
\hline BG433-M2 & M2 & & & 17.5 & Anglo-Saxon & Black Gate Newcastle & \\
\hline BG433-M3 & M3 & & & 17.5 & Anglo-Saxon & Black Gate Newcastle & \\
\hline BG498-M1 & M1 & & & 18.0 & Anglo-Saxon & Black Gate Newcastle & \\
\hline BG498-M2 & M2 & & & 17.5 & Anglo-Saxon & Black Gate Newcastle & \\
\hline BG498-M3 & M3 & & & 17.3 & Anglo-Saxon & Black Gate Newcastle & \\
\hline
\end{tabular}




\begin{tabular}{|c|c|c|c|c|c|c|c|}
\hline BG499-M1 & M1 & & & 17.4 & Anglo-Saxon & Black Gate Newcastle & \\
\hline BG499-M2 & M2 & & & 17.5 & Anglo-Saxon & Black Gate Newcastle & \\
\hline BG499-M3 & M3 & & & 18.0 & Anglo-Saxon & Black Gate Newcastle & \\
\hline BG534-M1 & M1 & & & 16.9 & Anglo-Saxon & Black Gate Newcastle & \\
\hline BG534-M2 & M2 & & & 17.1 & Anglo-Saxon & Black Gate Newcastle & \\
\hline BG534-M3 & M3 & & & 16.9 & Anglo-Saxon & Black Gate Newcastle & \\
\hline BG567-M1 & M1 & & & 17.1 & Anglo-Saxon & Black Gate Newcastle & \\
\hline BG567-M2 & M2 & & & 17.2 & Anglo-Saxon & Black Gate Newcastle & \\
\hline BG567-M3 & M3 & & & 16.4 & Anglo-Saxon & Black Gate Newcastle & \\
\hline BG576-M1 & M1 & & & 18.2 & Anglo-Saxon & Black Gate Newcastle & \\
\hline BG576-M2 & M2 & & & 17.7 & Anglo-Saxon & Black Gate Newcastle & \\
\hline BG576-M3 & M3 & & & 17.8 & Anglo-Saxon & Black Gate Newcastle & \\
\hline BG581-M1 & M1 & & & 17.4 & Anglo-Saxon & Black Gate Newcastle & \\
\hline BG581-M2 & M2 & & & 16.8 & Anglo-Saxon & Black Gate Newcastle & \\
\hline BG581-M3 & M3 & & & 17.1 & Anglo-Saxon & Black Gate Newcastle & \\
\hline BG591-M1 & M1 & & & 18.0 & Anglo-Saxon & Black Gate Newcastle & \\
\hline BG591-M2 & M2 & & & 17.7 & Anglo-Saxon & Black Gate Newcastle & \\
\hline BG591-M3 & M3 & & & 18.1 & Anglo-Saxon & Black Gate Newcastle & \\
\hline BG595-M1 & M1 & & & 18.0 & Anglo-Saxon & Black Gate Newcastle & \\
\hline BG626-M1 & M1 & & & 17.3 & Anglo-Saxon & Black Gate Newcastle & \\
\hline BG626-M2 & M2 & & & 17.1 & Anglo-Saxon & Black Gate Newcastle & \\
\hline BG626-M3 & M3 & & & 16.8 & Anglo-Saxon & Black Gate Newcastle & \\
\hline BG637-M1 & M1 & & & 18.5 & Anglo-Saxon & Black Gate Newcastle & \\
\hline BG637-M2 & M2 & & & 18.4 & Anglo-Saxon & Black Gate Newcastle & \\
\hline BG637-M3 & M3 & & & 18.0 & Anglo-Saxon & Black Gate Newcastle & \\
\hline BG659-M1 & M1 & & & 17.1 & Anglo-Saxon & Black Gate Newcastle & \\
\hline BG659-M2 & M2 & & & 17.1 & Anglo-Saxon & Black Gate Newcastle & \\
\hline BG659-M3 & M3 & & & 16.8 & Anglo-Saxon & Black Gate Newcastle & \\
\hline 209 & pM1 & 62 & 0.710959 & & Medieval & Blackfriars, Gloucester & \\
\hline 341 & $\mathrm{pM1R}$ & 59 & 0.714232 & 17.9 & Medieval & Blackfriars, Gloucester & \\
\hline 341 & pM1L & 56 & 0.714288 & 17.5 & Medieval & Blackfriars, Gloucester & \\
\hline 357 & pM2 & 56 & 0.711355 & & Medieval & Blackfriars, Gloucester & \\
\hline 77 & pM1 & 66 & 0.711971 & 17.6 & Medieval & Blackfriars, Gloucester & \\
\hline 89 & pM1 & 70 & 0.708877 & 16.8 & Medieval & Blackfriars, Gloucester & \\
\hline 1 (Boscombe) & pM2 & 63 & 0.707950 & 17.3 & Bronze Age & Boscombe and Amesbury & \\
\hline 1 (Boscombe) & M3 & 77 & 0.707964 & 16.8 & Bronze Age & Boscombe and Amesbury & \\
\hline 2 (Boscombe) & M3 & 189 & 0.709308 & 17.9 & Bronze Age & Boscombe and Amesbury & \\
\hline 2 (Boscombe) & pM2 & 108 & 0.709270 & 17.5 & Bronze Age & Boscombe and Amesbury & \\
\hline 25001 & pM2 & 55 & 0.709825 & 17.6 & Bronze Age & Boscombe and Amesbury & \\
\hline $25005 ?$ & $\mathrm{pM} 2$ & 55 & 0.709724 & 17.7 & Bronze Age & Boscombe and Amesbury & \\
\hline 3 (Boscombe) & pM2 & 55 & 0.707851 & 18.0 & Bronze Age & Boscombe and Amesbury & \\
\hline 3 (Boscombe) & M3 & 45 & 0.708003 & 17.6 & Bronze Age & Boscombe and Amesbury & \\
\hline 4 (Boscombe) & pM2 & 38 & 0.707837 & 17.8 & Bronze Age & Boscombe and Amesbury & \\
\hline 4 (Boscombe) & M3 & 50 & 0.708042 & 17.5 & Bronze Age & Boscombe and Amesbury & \\
\hline 5 (Boscombe) & pM2 & 52 & 0.708226 & 17.6 & Bronze Age & Boscombe and Amesbury & \\
\hline 5 (Boscombe) & M3 & 44 & 0.707910 & 17.5 & Bronze Age & Boscombe and Amesbury & \\
\hline 6 (Boscombe) & pM2 & 49 & 0.713436 & 17.5 & Bronze Age & Boscombe and Amesbury & \\
\hline 6 (Boscombe) & M3 & 59 & 0.711433 & 17.3 & Bronze Age & Boscombe and Amesbury & \\
\hline 7 (Boscombe) & M3 & 86 & 0.711743 & 17.0 & Bronze Age & Boscombe and Amesbury & \\
\hline 7 (Boscombe) & pM2 & 77 & 0.713093 & 16.9 & Bronze Age & Boscombe and Amesbury & \\
\hline BD 5353525217 & pM2 & 52 & 0.708222 & 18.8 & Bronze Age & Boscombe and Amesbury & \\
\hline BD 562406445 & M2 & 66 & 0.708085 & 17.5 & Bronze Age & Boscombe and Amesbury & \\
\hline BDLC 5353525010 & pM2 & 40 & 0.713519 & 17.5 & Bronze Age & Boscombe and Amesbury & \\
\hline BDLC 5353525010 & M3 & 45 & 0.711871 & 17.4 & Bronze Age & Boscombe and Amesbury & \\
\hline$W \times 1238^{*}$ & pM2 & & 0.708550 & 17.0 & Bronze Age & Boscombe and Amesbury & \\
\hline
\end{tabular}




\begin{tabular}{|c|c|c|c|c|c|c|c|}
\hline Wx1238* & M3 & & 0.709490 & 16.4 & Bronze Age & Boscombe and Amesbury & \\
\hline Wx1291A Archer* & M3 & & 0.709400 & 16.2 & Bronze Age & Boscombe and Amesbury & \\
\hline Wx1291A Archer* & $\mathrm{pM} 2$ & & 0.710340 & 16.2 & Bronze Age & Boscombe and Amesbury & \\
\hline CW 14 & M3 & 50 & 0.708023 & 17.8 & Bronze Age & Callis Wold & \\
\hline CW12 & pM1 & 92 & 0.707952 & 16.4 & Bronze Age & Callis Wold & \\
\hline CW13 & pM1 & 44 & 0.712010 & 18.5 & Bronze Age & Callis Wold & \\
\hline CW 275 & pM2 & 39 & 0.708896 & & Neolithic & Callis Wold & \\
\hline CW10 & $\mathrm{C}$ & 46 & 0.708437 & & Neolithic & Callis Wold & \\
\hline CW11 & $\mathrm{pM} 1$ & 55 & 0.709325 & 18.2 & Neolithic & Callis Wold & \\
\hline Cw9 & M2 & 49 & 0.709865 & 17.9 & Neolithic & Callis Wold & \\
\hline CBB-255-M2 & M2 & 46 & 0.710350 & 17.8 & Roman & Catterick & \\
\hline CBF-277-M3 & M3 & 114 & 0.713680 & 17.3 & Roman & Catterick & \\
\hline CBF-324-M3 & M3 & 131 & 0.709350 & 18.4 & Roman & Catterick & \\
\hline CBF-324-P2 & $\mathrm{pM} 2$ & 118 & 0.709230 & 18.5 & Roman & Catterick & \\
\hline CBF-422-M3 & M3 & 94 & 0.708990 & 17.1 & Roman & Catterick & \\
\hline CBF-475-M3 & M3 & 75 & 0.710340 & 17.9 & Roman & Catterick & \\
\hline CBF-564-M2 & M2 & 122 & 0.709250 & 17.1 & Roman & Catterick & \\
\hline CBF-632-M2 & M2 & 57 & 0.710530 & 18.0 & Roman & Catterick & \\
\hline CBF-678-M3 & M3 & 89 & 0.709110 & 16.9 & Roman & Catterick & \\
\hline CBF-679-M2 & M2 & 106 & 0.713670 & 17.7 & Roman & Catterick & \\
\hline CBF-687-M1 & M1 & 94 & 0.709460 & 19.0 & Roman & Catterick & \\
\hline CBF-709-M2 & M2 & 85 & 0.709030 & 18.1 & Roman & Catterick & \\
\hline CBF-746-M2 & M2 & 90 & 0.710950 & 17.1 & Roman & Catterick & \\
\hline CBF-746-M3 & M3 & 104 & 0.710530 & 17.5 & Roman & Catterick & \\
\hline CBF-756-P2 & $\mathrm{pM} 2$ & 74 & 0.710310 & 17.9 & Roman & Catterick & \\
\hline CBF-801-M3 & M3 & 153 & 0.709550 & 18.5 & Roman & Catterick & \\
\hline CBF-812-M1 & M1 & 64 & 0.709520 & 17.9 & Roman & Catterick & \\
\hline CBF-CX732-M3 & M3 & 50 & 0.710160 & 16.8 & Roman & Catterick & \\
\hline CBRI-037-M2 & M2 & 78 & 0.710160 & 17.8 & Roman & Catterick & \\
\hline CBRI-077-M3 & M3 & 84 & 0.710260 & 18.0 & Roman & Catterick & \\
\hline CBRI-136-M2 & M2 & 61 & 0.709970 & 17.3 & Roman & Catterick & \\
\hline CBRI-163-M2 & M2 & 96 & 0.710050 & 17.6 & Roman & Catterick & \\
\hline CBRI-166-M3 & M3 & 80 & 0.710470 & 17.4 & Roman & Catterick & \\
\hline CBRI-389-M1 & M1 & 50 & 0.709600 & 17.8 & Roman & Catterick & \\
\hline CBRI-484-M1 & M1 & 143 & 0.709470 & 17.7 & Roman & Catterick & \\
\hline CDS-PIV9-P2 & $\mathrm{pM} 2$ & 138 & 0.710260 & 18.1 & Roman & Catterick & \\
\hline CHP-941-M3 & M3 & 100 & 0.709790 & 17.0 & Roman & Catterick & \\
\hline CHP-942-M3 & M3 & 80 & 0.709520 & 18.0 & Roman & Catterick & \\
\hline CHP-942-P2 & $\mathrm{pM} 2$ & 87 & 0.709990 & 17.5 & Roman & Catterick & \\
\hline CHE 1051 & M1 & 141 & 0.709268 & 17.5 & C18th -19th & Chelsea & \\
\hline CHE 792 & $\mathrm{pM} 2$ & 118 & 0.706361 & 18.9 & C18th -19th & Chelsea & \\
\hline CHE 104 & M2 & 117 & 0.709087 & 17.7 & C18th -19th & Chelsea & Lower rainfall \\
\hline CHE 147 & $\mathrm{pM} 1$ & 83 & 0.708765 & 17.4 & C18th -19th & Chelsea & Lower rainfall \\
\hline CHE 161 & $\mathrm{pM} 2$ & 198 & 0.709310 & 17.0 & C18th -19th & Chelsea & Lower rainfall \\
\hline CHE 18 & $\mathrm{pM} 2$ & 277 & 0.709216 & 17.5 & C18th -19th & Chelsea & Lower rainfall \\
\hline CHE 19 & $\mathrm{pM} 2$ & 73 & 0.710898 & 18.6 & C18th -19th & Chelsea & Lower rainfall \\
\hline CHE 285 & M2 & 111 & 0.708974 & 18.8 & C18th -19th & Chelsea & Lower rainfall \\
\hline CHE 31 & M1 & 128 & 0.708932 & 17.5 & C18th -19th & Chelsea & Lower rainfall \\
\hline CHE 35 & pM2 & 137 & 0.709270 & 17.8 & C18th -19th & Chelsea & Lower rainfall \\
\hline CHE 39 & $\mathrm{pM} 2$ & 104 & 0.709318 & 17.4 & C18th -19th & Chelsea & Lower rainfall \\
\hline CHE 392 & $\mathrm{pM} 2$ & 154 & 0.709217 & 16.9 & C18th -19th & Chelsea & Lower rainfall \\
\hline CHE 552 & M2 & 83 & 0.709880 & 17.3 & C18th -19th & Chelsea & Lower rainfall \\
\hline CHE 654 & M2 & 125 & 0.709449 & 17.2 & C18th -19th & Chelsea & Lower rainfall \\
\hline CHE 697 & $\mathrm{pM} 2$ & 76 & 0.709202 & 17.2 & C18th -19th & Chelsea & Lower rainfall \\
\hline CHE 713 & M3 & 156 & 0.709468 & 17.3 & C18th -19th & Chelsea & Lower rainfall \\
\hline
\end{tabular}




\begin{tabular}{|c|c|c|c|c|c|c|c|}
\hline CHE 744 & pM2 & 163 & 0.709449 & 17.4 & C18th -19th & Chelsea & Lower rainfall \\
\hline CHE 750 & pM2 & 98 & 0.709163 & 17.6 & C18th -19th & Chelsea & Lower rainfall \\
\hline CHE 841 & M2 & 175 & 0.710357 & 16.7 & C18th -19th & Chelsea & Lower rainfall \\
\hline CHE 856 & pM2 & 175 & 0.709190 & 17.6 & C18th -19th & Chelsea & Lower rainfall \\
\hline CHE 918 & M2 & 135 & 0.709298 & 17.9 & C18th -19th & Chelsea & Lower rainfall \\
\hline Che 980 & $\mathrm{pM} 2$ & 90 & 0.708903 & 17.4 & C18th -19th & Chelsea & Lower rainfall \\
\hline CHE 990 & M2 & 39 & 0.709380 & 16.2 & C18th -19th & Chelsea & Lower rainfall \\
\hline CHE 994 & $\mathrm{pM} 2$ & 197 & 0.709295 & 17.9 & C18th -19th & Chelsea & Lower rainfall \\
\hline CHSU-5425-1 & M1 & & & 17.7 & Bronze Age & Clad Hallan & \\
\hline CGSU-0105-1 & no record & 226 & 0.709395 & 17.8 & Bronze Age & Clad Hallan & Higher rainfall \\
\hline $\mathrm{CHO}-2316$ & $\mathrm{C}$ & 223 & 0.709354 & 19.1 & Bronze Age & Clad Hallan & Higher rainfall \\
\hline $\mathrm{CHO} 1-2316$ & $\mathrm{M} 2$ & 295 & 0.709276 & 18.3 & Bronze Age & Clad Hallan & Higher rainfall \\
\hline CHO1-2638 & $\mathrm{M} 2$ & 299 & 0.709264 & 17.8 & Bronze Age & Clad Hallan & Higher rainfall \\
\hline $\mathrm{CHO}-2727$ & $\mathrm{C}$ & 217 & 0.709619 & 18.5 & Bronze Age & Clad Hallan & Higher rainfall \\
\hline $\mathrm{CHO} 1-2727$ & M2 & 201 & 0.709588 & 18.1 & Bronze Age & Clad Hallan & Higher rainfall \\
\hline CHSU-5087-1 & pM1? & 380 & 0.709359 & 18.6 & Bronze Age & Clad Hallan & Higher rainfall \\
\hline CHSU-5159-1 & M2 & 267 & 0.709541 & 18.6 & Bronze Age & Clad Hallan & Higher rainfall \\
\hline CHSU-5424-1-1 & M1 & 317 & 0.709355 & 18.7 & Bronze Age & Clad Hallan & Higher rainfall \\
\hline CHSU-5424-2-1 & M2 & 342 & 0.709356 & 18.6 & Bronze Age & Clad Hallan & Higher rainfall \\
\hline CHSU-5424-3-1 & M3 & 303 & 0.709528 & 18.4 & Bronze Age & Clad Hallan & Higher rainfall \\
\hline CNIP BA & $\mathrm{C}$ & 158 & 0.709460 & & Bronze Age & Cnip & \\
\hline CNIP A & $\mathrm{pM} 1$ & 101 & 0.710488 & 16.7 & Norse & Cnip & \\
\hline CNIP B & $\mathrm{dl} 1$ & 152 & 0.709595 & 17.8 & Norse & Cnip & \\
\hline CNIP C & $\mathrm{pM} 1$ & 210 & 0.709526 & & Norse & Cnip & \\
\hline CNIP D & $\mathrm{C}$ & 218 & 0.707932 & 17.2 & Norse & Cnip & \\
\hline CNIP D & pM1 & 169 & 0.707802 & 16.7 & Norse & Cnip & \\
\hline CNIP E & $\mathrm{pM} 1$ & 58 & 0.708575 & 17.3 & Norse & Cnip & \\
\hline CNIP F & $\mathrm{dl} 1$ & 165 & 0.709771 & & Norse & Cnip & \\
\hline CNIP G & $\mathrm{dl} 1$ & 417 & 0.710190 & & Norse & Cnip & \\
\hline CNIP-A-SK3-e & $\mathrm{M} 2$ & 232 & 0.709606 & 18.2 & Norse & Cnip & Higher rainfall \\
\hline CNIP-D-SK3-e & M2 & 320 & 0.709346 & 18.9 & Norse & cnip & Higher rainfall \\
\hline COV 1248 & M2 & 63 & 0.709620 & 16.3 & C18th -19th & Coventry & \\
\hline Cov 417 & pM1 & 94 & 0.710436 & 16.6 & C18th -19th & Coventry & \\
\hline Cov 434 & $\mathrm{pM} 2$ & 139 & 0.709778 & 16.1 & C18th -19th & Coventry & \\
\hline cov 50 & pM1 & 95 & 0.709972 & 17.0 & C18th -19th & Coventry & \\
\hline Cov 516 & M2 & 351 & 0.709183 & 16.8 & C18th -19th & Coventry & \\
\hline Cov 672 & M2 & 113 & 0.709940 & 16.3 & C18th -19th & Coventry & \\
\hline $\operatorname{cov} 77$ & $\mathrm{pM} 2$ & 91 & 0.710417 & 16.2 & C18th -19th & Coventry & \\
\hline Cov 808 & pM1 & 107 & 0.709974 & 16.6 & C18th -19th & Coventry & \\
\hline Cov 978 & pM1 & 142 & 0.709151 & 17.1 & C18th -19th & Coventry & \\
\hline $\operatorname{cov} 866$ & M2 & 109 & 0.710028 & 17.3 & C18th -19th & Coventry & \\
\hline CK 1225 & M2 & 107 & 0.710456 & 17.9 & Medieval & Cronk Keillane & \\
\hline CK 1226 & $\mathrm{M} 2$ & 113 & 0.712072 & 17.3 & Medieval & Cronk Keillane & \\
\hline CK 1234 & M2 & 74 & 0.710990 & 17.7 & Medieval & Cronk Keillane & \\
\hline CK 1236 & M2 & 127 & 0.710399 & 18.5 & Medieval & Cronk Keillane & \\
\hline CK 1581A & $\mathrm{M} 2$ & 121 & 0.708962 & 17.7 & Medieval & Cronk Keillane & \\
\hline CK 1769 & M2 & 146 & 0.709404 & 17.3 & Medieval & Cronk Keillane & \\
\hline CK 1781 & M2 & 67 & 0.709956 & 18.8 & Medieval & Cronk Keillane & \\
\hline AA-114-32 & M2 & 72 & 0.713270 & 17.6 & Medieval & Dore Abbey & Higher rainfall \\
\hline DRIF-10 & M2 & 67 & 0.709563 & 15.0 & Roman & Driffield/York & \\
\hline DRIF-15 & M3 & 73 & 0.714202 & 18.9 & Roman & Driffield/York & \\
\hline DRIF-16 & M3 & 71 & 0.709407 & 17.7 & Roman & Driffield/York & \\
\hline DRIF-33 & $\mathrm{M} 2$ & 131 & 0.708920 & 17.1 & Roman & Driffield/York & \\
\hline DRIF-35 & M2 & 67 & 0.709401 & 17.4 & Roman & Driffield/York & \\
\hline DRIF-37 & M2 & 42 & 0.708924 & 18.4 & Roman & Driffield/York & \\
\hline
\end{tabular}




\begin{tabular}{|c|c|c|c|c|c|c|c|}
\hline DRIF6-01 & $\mathrm{pM} 2$ & 79 & 0.710400 & 17.0 & Roman & Driffield/York & \\
\hline DRIF6-02 & $\mathrm{pM} 2$ & 113 & 0.711300 & 17.2 & Roman & Driffield/York & \\
\hline DRIF6-04 & pM2 & 57 & 0.710000 & 16.6 & Roman & Driffield/York & \\
\hline DRIF6-06 & $\mathrm{pM} 2$ & 68 & 0.709200 & 17.5 & Roman & Driffield/York & \\
\hline DRIF6-07 & $\mathrm{pM} 2$ & 62 & 0.709900 & 18.1 & Roman & Driffield/York & \\
\hline DRIF6-08 & pM2 & 55 & 0.711000 & 17.3 & Roman & Driffield/York & \\
\hline DRIF6-09 & $\mathrm{pM} 2$ & 43 & 0.712600 & 16.9 & Roman & Driffield/York & \\
\hline DRIF6-12 & $\mathrm{pM} 2$ & & & 18.1 & Roman & Driffield/York & \\
\hline DRIF6-14 & $\mathrm{pM} 2$ & 60 & 0.710900 & 16.7 & Roman & Driffield/York & \\
\hline DRIF6-15 & $\mathrm{pM} 2$ & 83 & 0.711400 & 16.5 & Roman & Driffield/York & \\
\hline DRIF6-17 & $\mathrm{pM} 2$ & 51 & 0.710300 & 17.1 & Roman & Driffield/York & \\
\hline DRIF6-18 & $\mathrm{pM} 2$ & 62 & 0.709100 & 18.6 & Roman & Driffield/York & \\
\hline DRIF6-19 & $\mathrm{pM} 2$ & 72 & 0.709400 & 18.7 & Roman & Driffield/York & \\
\hline DRIF6-20 & $\mathrm{pM} 2$ & 34 & 0.711400 & 16.7 & Roman & Driffield/York & \\
\hline DRIF6-21 & $\mathrm{pM} 2$ & 90 & 0.709400 & 19.8 & Roman & Driffield/York & \\
\hline DRIF6-22 & $\mathrm{pM} 2$ & 104 & 0.709200 & 18.6 & Roman & Driffield/York & \\
\hline DRIF6-23 & $\mathrm{pM} 2$ & 65 & 0.710900 & 16.7 & Roman & Driffield/York & \\
\hline DRIF6-24 & $\mathrm{pM} 2$ & 56 & 0.708500 & 14.7 & Roman & Driffield/York & \\
\hline $\mathrm{DH} 1$ & 12 & 68 & 0.710201 & 17.9 & Neolithic & Duggleby Howe & \\
\hline $\mathrm{DH} 2$ & M2 & 86 & 0.709570 & & Neolithic & Duggleby Howe & \\
\hline $\mathrm{DH} 3$ & M2 & 50 & 0.709138 & 17.5 & Neolithic & Duggleby Howe & \\
\hline $\mathrm{DH} 4$ & $\mathrm{pM} 1$ & 44 & 0.708594 & 18.9 & Neolithic & Duggleby Howe & \\
\hline DH5 & $\mathrm{pM} 2$ & 49 & 0.709997 & 17.7 & Neolithic & Duggleby Howe & \\
\hline DH6 & M2 & 72 & 0.709349 & 17.8 & Neolithic & Duggleby Howe & \\
\hline $\mathrm{DH} 7$ & $\mathrm{pM1}$ & 41 & 0.709849 & 18.3 & Neolithic & Duggleby Howe & \\
\hline G318 & pM1 & 56 & 0.708227 & 17.9 & Roman & Eagle Hotel & \\
\hline G319 & $\mathrm{pM} 1$ & 91 & 0.708482 & 18.9 & Roman & Eagle Hotel & \\
\hline G326 & $\mathrm{pM} 1$ & 80 & 0.708629 & 18.2 & Roman & Eagle Hotel & \\
\hline G339A & M3 & 93 & 0.709147 & 18.2 & Roman & Eagle Hotel & \\
\hline G339B & $\mathrm{pM} 2$ & 79 & 0.709310 & 18.3 & Roman & Eagle Hotel & \\
\hline Morgan-1 & $\mathrm{pM} ? 1 / 2$ & 78 & 0.710755 & 18.3 & Anglo-Saxon & Easington & \\
\hline Morgan-2 & M2 & 74 & 0.710714 & 18.1 & Anglo-Saxon & Easington & \\
\hline Morgan-3 & $\mathrm{M} 2$ & 112 & 0.712142 & 18.0 & Anglo-Saxon & Easington & \\
\hline Morgan-4 & M2 & 98 & 0.709479 & 18.1 & Anglo-Saxon & Easington & \\
\hline Morgan-5 & M2 & 119 & 0.710144 & 17.9 & Anglo-Saxon & Easington & \\
\hline EAS-051 & $\mathrm{pM} 2$ & 77 & 0.708638 & 17.3 & Anglo-Saxon & Eastbourne & \\
\hline EAS-057 & $\mathrm{pM} 2$ & 61 & 0.709669 & 17.6 & Anglo-Saxon & Eastbourne & \\
\hline EAS-061 & $\mathrm{pM} 2$ & 80 & 0.708819 & 18.4 & Anglo-Saxon & Eastbourne & \\
\hline EAS-064 & $\mathrm{pM} 2$ & 65 & 0.711291 & 18.2 & Anglo-Saxon & Eastbourne & \\
\hline EAS-067 & $\mathrm{pM} 2$ & 66 & 0.709193 & 18.1 & Anglo-Saxon & Eastbourne & \\
\hline EAS-111 & $\mathrm{pM} 2$ & 43 & 0.708818 & 18.2 & Anglo-Saxon & Eastbourne & \\
\hline EAS-157 & $\mathrm{pM} 2$ & 94 & 0.708640 & 18.7 & Anglo-Saxon & Eastbourne & \\
\hline EAS-190 & M2 & 69 & 0.709084 & 18.6 & Anglo-Saxon & Eastbourne & \\
\hline EAS-233 & $\mathrm{pM} 2$ & 49 & 0.709030 & 18.5 & Anglo-Saxon & Eastbourne & \\
\hline EAS-264 & $\mathrm{pM} 2$ & 63 & 0.710084 & 17.9 & Anglo-Saxon & Eastbourne & \\
\hline EAS-270 & $\mathrm{pM} 2$ & 60 & 0.710514 & 17.3 & Anglo-Saxon & Eastbourne & \\
\hline EAS-309 & $\mathrm{pM} 2$ & 52 & 0.710002 & 17.3 & Anglo-Saxon & Eastbourne & \\
\hline EAS-355 & $\mathrm{pM} 2$ & 73 & 0.709336 & 17.1 & Anglo-Saxon & Eastbourne & \\
\hline EAS-381 & $\mathrm{pM} 2$ & 77 & 0.708681 & 18.2 & Anglo-Saxon & Eastbourne & \\
\hline EAS-481 & $\mathrm{pM} 2$ & 73 & 0.710079 & 17.7 & Anglo-Saxon & Eastbourne & \\
\hline EAS-650 & $\mathrm{pM} 2$ & 81 & 0.709080 & 18.1 & Anglo-Saxon & Eastbourne & \\
\hline EAS-681 & $\mathrm{pM} 2$ & 89 & 0.708898 & 18.0 & Anglo-Saxon & Eastbourne & \\
\hline EAS-753 & $\mathrm{pM} 2$ & 95 & 0.708683 & 18.8 & Anglo-Saxon & Eastbourne & \\
\hline EAS-796 & $\mathrm{pM} 2$ & 89 & 0.708835 & 18.2 & Anglo-Saxon & Eastbourne & \\
\hline EMP -039 & no record & 83 & 0.709593 & 16.3 & Anglo-Saxon & Empingham & Lower rainfall \\
\hline
\end{tabular}




\begin{tabular}{|c|c|c|c|c|c|c|c|}
\hline Emp -046 & no record & 35 & 0.709677 & 17.0 & Anglo-Saxon & Empingham & Lower rainfall \\
\hline EMP -049 & no record & 55 & 0.710481 & 17.2 & Anglo-Saxon & Empingham & Lower rainfall \\
\hline Emp -081 & no record & 55 & 0.709476 & 16.9 & Anglo-Saxon & Empingham & Lower rainfall \\
\hline EMP -110 & no record & 45 & 0.710113 & 16.8 & Anglo-Saxon & Empingham & Lower rainfall \\
\hline EMP- 115 & no record & 51 & 0.710488 & 17.1 & Anglo-Saxon & Empingham & Lower rainfall \\
\hline EMP -119 & no record & 58 & 0.709567 & 17.1 & Anglo-Saxon & Empingham & Lower rainfall \\
\hline EMP-003 & no record & & & 16.4 & Anglo-Saxon & Empingham & Lower rainfall \\
\hline EMP-026 & no record & 35 & 0.709478 & 17.1 & Anglo-Saxon & Empingham & Lower rainfall \\
\hline EMP-030 & $\mathrm{pM} 2$ & 63 & 0.709514 & & Anglo-Saxon & Empingham & Lower rainfall \\
\hline EMP-031B & no record & & & 16.1 & Anglo-Saxon & Empingham & Lower rainfall \\
\hline EMP-031C & no record & & & 17.7 & Anglo-Saxon & Empingham & Lower rainfall \\
\hline EMP-037 & no record & & & 17.5 & Anglo-Saxon & Empingham & Lower rainfall \\
\hline EMP-050 & no record & 82 & 0.710030 & 17.3 & Anglo-Saxon & Empingham & Lower rainfall \\
\hline Emp-094 & no record & 76 & 0.710463 & 16.9 & Anglo-Saxon & Empingham & Lower rainfall \\
\hline EMP-095 & no record & & & 17.0 & Anglo-Saxon & Empingham & Lower rainfall \\
\hline FCR03-M1 & M1 & & & 17.4 & Anglo-Saxon & Filingham & \\
\hline FCR03-M2 & M2 & & & 16.7 & Anglo-Saxon & Filingham & \\
\hline FCR03-M3 & M3 & & & 17.4 & Anglo-Saxon & Filingham & \\
\hline FCR04-M1 & M1 & & & 17.7 & Anglo-Saxon & Filingham & \\
\hline FCR04-M2 & M2 & & & 17.0 & Anglo-Saxon & Filingham & \\
\hline FCR04-M3 & M3 & & & 17.5 & Anglo-Saxon & Filingham & \\
\hline Gals-93 & pM1 & 71 & 0.713033 & 16.1 & Iron Age & Galson & \\
\hline Gals-96 & pM1 & 337 & 0.709345 & 17.8 & Iron Age & Galson & \\
\hline Gals-II & pM1 & 233 & 0.709417 & 17.0 & Iron Age & Galson & \\
\hline Gals-IV & $\mathrm{pM} 1$ & 82 & 0.711860 & 17.0 & Iron Age & Galson & \\
\hline Gals-74 & C & 174 & 0.709379 & 16.9 & Iron Age & Galston & \\
\hline GLR1103 & M3 & 114 & 0.709440 & 18.4 & Roman & Gloucester & \\
\hline GLR1127 & M3 & 121 & 0.709669 & 17.2 & Roman & Gloucester & \\
\hline GLR1131 & M3 & 133 & 0.711428 & 17.1 & Roman & Gloucester & \\
\hline GLR1181 & M2 & 76 & 0.710574 & 17.6 & Roman & Gloucester & \\
\hline GLR1216 & M3 & 93 & 0.709407 & 19.2 & Roman & Gloucester & \\
\hline GLR1238 & M3 & 52 & 0.708912 & 17.1 & Roman & Gloucester & \\
\hline GLR1328 & M3 & 57 & 0.710559 & 18.0 & Roman & Gloucester & \\
\hline GLR1340 & M2 & 104 & 0.709581 & 17.9 & Roman & Gloucester & \\
\hline GLR1360 & M2 & 133 & 0.708996 & 19.0 & Roman & Gloucester & \\
\hline GLR1364 & M3 & 104 & 0.711380 & 18.2 & Roman & Gloucester & \\
\hline GLR1518 & M2 & 72 & 0.712980 & 17.9 & Roman & Gloucester & \\
\hline GLR1520 & M3 & 130 & 0.709707 & 18.6 & Roman & Gloucester & \\
\hline GLR1539 & M3 & 66 & 0.708780 & 17.5 & Roman & Gloucester & \\
\hline GLR1541 & M3 & 51 & 0.712175 & 17.1 & Roman & Gloucester & \\
\hline GLR1544 & M3 & 102 & 0.709944 & 18.2 & Roman & Gloucester & \\
\hline GLR1546 & M3 & 177 & 0.710864 & 19.1 & Roman & Gloucester & \\
\hline GLR1553 & M3 & 84 & 0.709243 & 17.4 & Roman & Gloucester & \\
\hline GLR1560 & M3 & 114 & 0.709461 & 18.7 & Roman & Gloucester & \\
\hline GLR1561 & M3 & 67 & 0.713443 & 18.7 & Roman & Gloucester & \\
\hline GLR1565 & M2 & 104 & 0.709748 & 19.0 & Roman & Gloucester & \\
\hline GLR1596 & M2 & 105 & 0.709042 & 17.7 & Roman & Gloucester & \\
\hline $44-77-146$ & $\mathrm{pM} 2$ & 70 & 0.710900 & 16.0 & Roman & Gloucester & \\
\hline $44-77-146$ & M3 & 54 & 0.711010 & 15.2 & Roman & Gloucester & \\
\hline P16379 & $\mathrm{pM} 1$ & 86 & 0.710443 & 17.9 & Bronze Age & Great Orme & Higher rainfall \\
\hline 3061 & pM2? & 115 & 0.708810 & 17.4 & C18th -19th & Greenwich & \\
\hline GRIS & M2 & 66 & 0.710723 & 17.2 & Bronze Age & Gristhorpe & \\
\hline HAUG-1 & M2 & 307 & 0.709340 & 18.0 & Neolithic & Haugabost & Higher rainfall \\
\hline HFRD-140 & M2 & 90 & 0.712377 & & Medieval & Hereford & \\
\hline HFRD-1798 & M2 & 64 & 0.712861 & & Medieval & Hereford & \\
\hline
\end{tabular}




\begin{tabular}{|c|c|c|c|c|c|c|c|}
\hline HFRD-221 & M2 & 92 & 0.709334 & & Medieval & Hereford & \\
\hline HFRD-2374 & M2 & 138 & 0.712372 & & Medieval & Hereford & \\
\hline HFRD-2656 & M2 & 87 & 0.713255 & & Medieval & Hereford & \\
\hline HFRD-3265 & M2 & 44 & 0.712754 & & Medieval & Hereford & \\
\hline HFRD-341 & M2 & 53 & 0.712866 & & Medieval & Hereford & \\
\hline HFRD-3661 & M2 & 66 & 0.712479 & & Medieval & Hereford & \\
\hline HFRD-869 & M2 & 46 & 0.712711 & & Medieval & Hereford & \\
\hline HFRD-869 & M2 & 42 & 0.714008 & & Medieval & Hereford & \\
\hline HFRD-905 & M2 & 62 & 0.712523 & & Medieval & Hereford & \\
\hline HFRD-1517 & M2 & 70 & 0.712482 & 18.5 & Medieval & Hereford & Higher rainfall \\
\hline HFRD-1774 & M2 & 124 & 0.710539 & 17.7 & Medieval & Hereford & Higher rainfall \\
\hline HFRD-2006 & M2 & 55 & 0.712607 & 17.9 & Medieval & Hereford & Higher rainfall \\
\hline HFRD-3850 & M2 & 66 & 0.713292 & 17.8 & Medieval & Hereford & Higher rainfall \\
\hline HFRD-410 & M2 & 111 & 0.711875 & 17.7 & Medieval & Hereford & Higher rainfall \\
\hline HFRD-713 & M2 & 57 & 0.712857 & 18.6 & Medieval & Hereford & Higher rainfall \\
\hline HFRD-911 & M2 & 60 & 0.713023 & 17.9 & Medieval & Hereford & Higher rainfall \\
\hline HFRD-959 & M2 & 91 & 0.712951 & 16.9 & Medieval & Hereford & Higher rainfall \\
\hline HPCS-100 & $\mathrm{dC} 1$ & 140 & 0.709085 & 17.4 & Iron Age & High Pasture Cave & \\
\hline HPCS-101 & M1 & 172 & 0.709317 & 17.7 & Iron Age & High Pasture Cave & Higher rainfall \\
\hline HPCS-102 & M2 & 128 & 0.709081 & 17.6 & Iron Age & High Pasture Cave & Higher rainfall \\
\hline HPCS-103 & M3 & 151 & 0.709061 & 17.5 & Iron Age & High Pasture Cave & Higher rainfall \\
\hline Inch & M1 & 75 & 0.709928 & 18.2 & Bronze Age & Inchmarnock & \\
\hline KCC-Roman-2 & no record & & & 18.2 & Roman & Ketton & \\
\hline KCC-Roman-3 & no record & & & 17.5 & Roman & Ketton & \\
\hline KCC 9857 & deciduous & 44 & 0.709553 & 18.2 & Anglo-Saxon & Ketton & \\
\hline KCC 9871 & deciduous & 86 & 0.709358 & 17.5 & Anglo-Saxon & Ketton & \\
\hline KCC 007 & adult & 66 & 0.708966 & 16.3 & Anglo-Saxon & Ketton & Lower rainfall \\
\hline KCC 014 & adult & 153 & 0.712663 & 17.2 & Anglo-Saxon & Ketton & Lower rainfall \\
\hline KCC 017 & adult & 58 & 0.709505 & 16.8 & Anglo-Saxon & Ketton & Lower rainfall \\
\hline KCC 047 & adult & 72 & 0.709372 & 16.2 & Anglo-Saxon & Ketton & Lower rainfall \\
\hline KCC 049 & adult & & & 17.2 & Anglo-Saxon & Ketton & Lower rainfall \\
\hline KCC 058 & adult & 67 & 0.709392 & 16.8 & Anglo-Saxon & Ketton & Lower rainfall \\
\hline KCC 064 & adult & 105 & 0.711407 & 16.4 & Anglo-Saxon & Ketton & Lower rainfall \\
\hline KCC 065 & adult & 60 & 0.710489 & 15.5 & Anglo-Saxon & Ketton & Lower rainfall \\
\hline $\mathrm{KCC} 066$ & adult & 81 & 0.709375 & 17.3 & Anglo-Saxon & Ketton & Lower rainfall \\
\hline KCC 067 & adult & 123 & 0.710261 & 17.2 & Anglo-Saxon & Ketton & Lower rainfall \\
\hline KCC 9813 & $\begin{array}{l}\text { permanent } \\
\text { (child) }\end{array}$ & 44 & 0.709460 & 17.2 & Anglo-Saxon & Ketton & Lower rainfall \\
\hline KCC 9832 & $\begin{array}{l}\text { permanent } \\
\text { (child) }\end{array}$ & 51 & 0.709244 & 16.6 & Anglo-Saxon & Ketton & Lower rainfall \\
\hline KCC 9834 & adult & 62 & 0.709213 & 16.7 & Anglo-Saxon & Ketton & Lower rainfall \\
\hline KCC 9840 & $\begin{array}{l}\text { permanent } \\
\text { (child) }\end{array}$ & 79 & 0.709564 & 16.2 & Anglo-Saxon & Ketton & Lower rainfall \\
\hline KCC 9852 & adult & 60 & 0.709535 & 16.8 & Anglo-Saxon & Ketton & Lower rainfall \\
\hline KCC 9854 & adult & 126 & 0.709835 & 16.1 & Anglo-Saxon & Ketton & Lower rainfall \\
\hline KCC 9855 & adult & 75 & 0.710566 & 16.9 & Anglo-Saxon & Ketton & Lower rainfall \\
\hline KCC 9856 & adult & 97 & 0.710997 & 18.3 & Anglo-Saxon & Ketton & Lower rainfall \\
\hline KCC 986 & $\begin{array}{l}\text { permanent } \\
\text { (child) }\end{array}$ & 104 & 0.709275 & 16.8 & Anglo-Saxon & Ketton & Lower rainfall \\
\hline KCC 9863 & adult & 86 & 0.710267 & 17.2 & Anglo-Saxon & Ketton & Lower rainfall \\
\hline KCC $9868 a$ & $\begin{array}{l}\text { permanent } \\
\text { (child) }\end{array}$ & 53 & 0.709588 & 17.4 & Anglo-Saxon & Ketton & Lower rainfall \\
\hline KCC 98 68b & $\begin{array}{l}\text { permanent } \\
\text { (child) }\end{array}$ & 58 & 0.709878 & 17.2 & Anglo-Saxon & Ketton & Lower rainfall \\
\hline KCC 989 & adult & 69 & 0.709349 & 16.1 & Anglo-Saxon & Ketton & Lower rainfall \\
\hline K1 & M1 & 126 & 0.709206 & 17.2 & Iron Age & Kilpheder & \\
\hline $\mathrm{K} 2$ & M2 & 130 & 0.708876 & 17.8 & Iron Age & Kilpheder & \\
\hline KH49-M1 & M1 & & & 18.2 & Anglo-Saxon & Kilton Hill & \\
\hline KH49-M2 & M2 & & & 17.8 & Anglo-Saxon & Kilton Hill & \\
\hline
\end{tabular}




\begin{tabular}{|c|c|c|c|c|c|c|}
\hline KH49-M3 & M3 & & & 18.0 & Anglo-Saxon & Kilton Hill \\
\hline KH54-M1 & M1 & & & 17.7 & Anglo-Saxon & Kilton Hill \\
\hline KH54-M2 & M2 & & & 17.6 & Anglo-Saxon & Kilton Hill \\
\hline KH54-M3 & M3 & & & 17.4 & Anglo-Saxon & Kilton Hill \\
\hline CIKNO-08-SK1403 & M1 & 58 & 0.709620 & 18.7 & Bronze Age & $\begin{array}{l}\text { Kings Hill } \\
\text { North/Cirencester }\end{array}$ \\
\hline CIKNO-08-SK1403 & PM2 & 44 & 0.708939 & 18.1 & Bronze Age & $\begin{array}{l}\text { Kings Hill } \\
\text { North/Cirencester }\end{array}$ \\
\hline CIKNO-08-SK8656 & M2 & 36 & 0.708720 & 17.5 & Bronze Age & $\begin{array}{l}\text { Kings Hill } \\
\text { North/Cirencester }\end{array}$ \\
\hline CIKNO-08-SK8656 & PM2 & 37 & 0.708566 & 17.1 & Bronze Age & $\begin{array}{l}\text { Kings Hill } \\
\text { North/Cirencester }\end{array}$ \\
\hline Ay21-0012 & $\mathrm{pM} 2$ & 103 & 0.708173 & 18.2 & Roman & Lankhills \\
\hline Ay21-0084 & M2 & 79 & 0.708599 & 18.8 & Roman & Lankhills \\
\hline Ay21-0119 & M2 & 100 & 0.708707 & 19.5 & Roman & Lankhills \\
\hline Ay21-0212 & $\mathrm{pM} 2$ & 66 & 0.708712 & 18.3 & Roman & Lankhills \\
\hline AY21-0271 & M2 & 139 & 0.710227 & 19.4 & Roman & Lankhills \\
\hline AY21-0281 & M2 & 92 & 0.711497 & 16.8 & Roman & Lankhills \\
\hline AY21-0435 & $\mathrm{M} 2$ & 53 & 0.708946 & 17.5 & Roman & Lankhills \\
\hline Ay21-0489 & M2 & 72 & 0.711242 & 17.8 & Roman & Lankhills \\
\hline AY21-0566 & M2 & 169 & 0.709516 & 18.8 & Roman & Lankhills \\
\hline Ay21-0661 & $\mathrm{pM} 1$ & 54 & 0.708481 & 18.1 & Roman & Lankhills \\
\hline Ay21-0683 & M3 & 132 & 0.709382 & 18.9 & Roman & Lankhills \\
\hline AY21-0776 & M2 & 86 & 0.709647 & 17.8 & Roman & Lankhills \\
\hline AY21-0806 & $\mathrm{pM} 2$ & 88 & 0.708734 & 19.3 & Roman & Lankhills \\
\hline AY21-0812 & $\mathrm{M} 2$ & 128 & 0.708674 & 19.0 & Roman & Lankhills \\
\hline Ay21-0861M3 & M3 & 54 & 0.709811 & 18.0 & Roman & Lankhills \\
\hline Ay21-0861P2 & $\mathrm{pM} 2$ & 54 & 0.709822 & 18.2 & Roman & Lankhills \\
\hline AY21-0862 & M2 & 118 & 0.708197 & 17.8 & Roman & Lankhills \\
\hline Ay21-0874 & M2 & 81 & 0.708752 & 18.0 & Roman & Lankhills \\
\hline AY21-0926 & M2 & 104 & 0.708615 & 18.3 & Roman & Lankhills \\
\hline Ay21-0932 & $\mathrm{M} 2$ & 67 & 0.708414 & 17.9 & Roman & Lankhills \\
\hline Ay21-1026 & M1 & 78 & 0.708504 & 17.9 & Roman & Lankhills \\
\hline Ay21-1084 & M2 & 105 & 0.709169 & 18.2 & Roman & Lankhills \\
\hline AY21-1091 & M2 & 105 & 0.709081 & 18.1 & Roman & Lankhills \\
\hline AY21-1094 & M2 & 42 & 0.708343 & 17.0 & Roman & Lankhills \\
\hline AY21-1114 & M2 & 121 & 0.708862 & 19.0 & Roman & Lankhills \\
\hline Ay21-1119 & M2 & 87 & 0.709416 & 15.8 & Roman & Lankhills \\
\hline Ay21-1133 & $\mathrm{M} 1$ & 51 & 0.709190 & 18.5 & Roman & Lankhills \\
\hline AY21-1134 & $\mathrm{pM} 2$ & 65 & 0.708624 & 18.0 & Roman & Lankhills \\
\hline Ay21-1197 & $\mathrm{pM} 2$ & 59 & 0.710993 & 17.8 & Roman & Lankhills \\
\hline AY21-1207 & M2 & 108 & 0.708898 & 17.9 & Roman & Lankhills \\
\hline AY21-1227 & M2 & 73 & 0.708304 & 18.3 & Roman & Lankhills \\
\hline AY21-1244 & M2 & 61 & 0.708586 & 18.4 & Roman & Lankhills \\
\hline Ay21-1271 & M2 & 67 & 0.708331 & 17.7 & Roman & Lankhills \\
\hline AY21-1277 & M2 & 87 & 0.711478 & 18.2 & Roman & Lankhills \\
\hline Ay21-1289 & M2 & 58 & 0.708723 & 18.1 & Roman & Lankhills \\
\hline AY21-1517 & $\mathrm{pM} 2$ & 81 & 0.709040 & 18.7 & Roman & Lankhills \\
\hline Ay21-1697 & $\mathrm{M} 2$ & 95 & 0.708971 & 18.8 & Roman & Lankhills \\
\hline Ay21-1761 & M2 & 80 & 0.708575 & 18.3 & Roman & Lankhills \\
\hline Ay21-1870 & M2 & 79 & 0.708716 & 17.6 & Roman & Lankhills \\
\hline AY21-1894 & $\mathrm{pM} 2$ & 46 & 0.711794 & 18.1 & Roman & Lankhills \\
\hline Lankhills 053 & $\mathrm{pM}$ & 81 & 0.708500 & 17.9 & Roman & Lankhills \\
\hline Lankhills 055 & $\mathrm{pM}$ & 120 & 0.709200 & 16.4 & Roman & Lankhills \\
\hline Lankhills 057 & $\mathrm{pM}$ & 136 & 0.708700 & 17.3 & Roman & Lankhills \\
\hline Lankhills 117 & $\mathrm{pM}$ & 106 & 0.708400 & 17.7 & Roman & Lankhills \\
\hline Lankhills 357 & $\mathrm{C}$ & 206 & 0.709100 & 16.2 & Roman & Lankhills \\
\hline Lankhills 382 & M2 & 107 & 0.708600 & 18.5 & Roman & Lankhills \\
\hline
\end{tabular}




\begin{tabular}{|c|c|c|c|c|c|c|c|}
\hline Lankhills 398 & $\mathrm{pM}$ & 82 & 0.708500 & 17.0 & Roman & Lankhills & \\
\hline Lankhills 437 & M1 & 57 & 0.708400 & 17.7 & Roman & Lankhills & \\
\hline Lankhills 443 & M2 & 75 & 0.708300 & 18.0 & Roman & Lankhills & \\
\hline Ay21-0118 & dl1 & 77 & 0.708848 & 19.1 & Roman & Lankhills & \\
\hline Lankhills 013 & $\mathrm{C}$ & 255 & 0.706400 & 15.8 & Roman & Lankhills & \\
\hline Lankhills 081 & M2 & 92 & 0.709300 & 14.7 & Roman & Lankhills & \\
\hline Lankhills 322 & M & 76 & 0.711600 & 17.3 & Roman & Lankhills & \\
\hline Lankhills 323 & $\mathrm{dM} 2$ & 146 & 0.708600 & 18.9 & Roman & Lankhills & \\
\hline Lankhills 326 & M3? & 105 & 0.708700 & 18.2 & Roman & Lankhills & \\
\hline Lankhills 333 & $\mathrm{dM} 2$ & 84 & 0.708600 & 18.8 & Roman & Lankhills & \\
\hline Lankhills 351 & $\mathrm{C}$ & 139 & 0.709000 & 16.0 & Roman & Lankhills & \\
\hline Lankhills 426 & $\mathrm{pM}$ & 123 & 0.709400 & 15.1 & Roman & Lankhills & \\
\hline SHY004 & M2? & 75 & 0.708517 & 18.2 & C16th & London/Tilney & \\
\hline $\mathrm{B} 2 \mathrm{~S} 2$ & no record & 234 & 0.709429 & & Bronze Age & Lunan bay & \\
\hline Lund-1 & M2 & 88 & 0.708816 & 17.3 & Bronze Age & Lundin Links & \\
\hline SK1a & $\mathrm{C}$ & 190 & 0.709890 & 17.7 & Roman & Mangotsfield, Bristol & \\
\hline Sk1b & $\mathrm{C}$ & & 0.710165 & 17.6 & Roman & Mangotsfield, Bristol & \\
\hline SK2a & pM2 & 37 & 0.709949 & 17.7 & Roman & Mangotsfield, Bristol & \\
\hline $\mathrm{SK} 2 \mathrm{~b}$ & $\mathrm{C}$ & & 0.709795 & 17.1 & Roman & Mangotsfield, Bristol & \\
\hline MH04-897 & pM1 & 419 & 0.709407 & 17.7 & Iron Age & Mine Howe & Higher rainfall \\
\hline MH05-1861 & M2 & 406 & 0.709403 & 17.6 & Iron Age & Mine Howe & Higher rainfall \\
\hline F23E & $\mathrm{P} 1$ & 85 & 0.708037 & 18.5 & Bronze Age & Monkton & \\
\hline F23A & M1 & 71 & 0.708776 & 18.6 & Neolithic & Monkton & \\
\hline $\mathrm{F} 23 \mathrm{~B}$ & pM1 & 57 & 0.709277 & 18.4 & Neolithic & Monkton & \\
\hline $\mathrm{F} 23 \mathrm{C}$ & pM2 & 55 & 0.710072 & 18.8 & Neolithic & Monkton & \\
\hline$F 23 D$ & $\mathrm{C}$ & 54 & 0.708965 & 19.6 & Neolithic & Monkton & \\
\hline Mull 1 & M1 & 124 & 0.709173 & 18.1 & Bronze Age & Mull & \\
\hline $\mathrm{PH}$ SK 16 & M2 & 341 & 0.709160 & 17.0 & Medieval & Parliament House & \\
\hline PH SK 45 & M2 & 219 & 0.709690 & 17.1 & Medieval & Parliament House & \\
\hline PH SK 58 & M2 & 91 & 0.710060 & 18.8 & Medieval & Parliament House & \\
\hline PH SK 60 & M2 & 87 & 0.709830 & 17.3 & Medieval & Parliament House & \\
\hline PH SK 71 & M2 & 77 & 0.716460 & 17.4 & Medieval & Parliament House & \\
\hline $\mathrm{PH}$ SK 92 & M2 & 92 & 0.709050 & 17.7 & Medieval & Parliament House & \\
\hline Rame-1 & pM2 & 120 & 0.711062 & 17.2 & Bronze Age & Rameldry Farm & \\
\hline G295 & pM1 & 157 & 0.708980 & 17.9 & Medieval & Repton & \\
\hline G511 & pM2 & 110 & 0.709600 & 17.9 & Medieval & Repton & \\
\hline G529 & M2 & 113 & 0.711850 & 16.4 & Medieval & Repton & \\
\hline G97 & $\mathrm{C}$ & 54 & 0.711950 & 18.9 & Medieval & Repton & \\
\hline$\times 17$ & pM1 & 72 & 0.712000 & 16.7 & Medieval & Repton & \\
\hline$\times 23$ & $\mathrm{pM} 1$ & 69 & 0.710510 & 17.1 & Medieval & Repton & \\
\hline $\mathrm{x} 3$ & pM2 & 43 & 0.710960 & 18.3 & Medieval & Repton & \\
\hline$\times 70$ & pM1 & 75 & 0.709900 & 17.3 & Medieval & Repton & \\
\hline 1974-121-SK1 & $\mathrm{pM} 2$ & & 0.708747 & 17.0 & Medieval & Riccall & \\
\hline 1974-121-SK18 & pM2 & & 0.710968 & 18.2 & Medieval & Riccall & \\
\hline 1974-121-SK23 & pM2 & & 0.709789 & 17.5 & Medieval & Riccall & \\
\hline 1974-121-SK4 & pM2 & & 0.709891 & 18.4 & Medieval & Riccall & \\
\hline 1985.11.SK11/SK16 & pM1 & 143 & 0.709988 & 18.4 & Medieval & Riccall & \\
\hline 1985.11.SK15 & pM1 & 78 & 0.710535 & 18.4 & Medieval & Riccall & \\
\hline 1985.11.SK18 & pM1 & 87 & 0.710939 & 18.4 & Medieval & Riccall & \\
\hline 1985.11.SK22 & pM1 & 74 & 0.710837 & 17.8 & Medieval & Riccall & \\
\hline 1985.11.SK7 & pM1 & 75 & 0.711648 & 17.8 & Medieval & Riccall & \\
\hline 1985.11.SK9 & pM1 & 85 & 0.711261 & 18.4 & Medieval & Riccall & \\
\hline 1985-11-SK1 & pM2 & & 0.709935 & 17.7 & Medieval & Riccall & \\
\hline 1985-11-SK9 & pM2 & & 0.710600 & 18.3 & Medieval & Riccall & \\
\hline RING008 & M2 & 42 & 0.708707 & 18.7 & Anglo-Saxon & Ringlemere & \\
\hline
\end{tabular}




\begin{tabular}{|c|c|c|c|c|c|c|c|}
\hline RING018 & M2 & 125 & 0.708846 & 17.6 & Anglo-Saxon & Ringlemere & \\
\hline RING025 & M2 & 98 & 0.709052 & 18.2 & Anglo-Saxon & Ringlemere & \\
\hline RING030 & M2 & 91 & 0.709069 & 18.4 & Anglo-Saxon & Ringlemere & \\
\hline RING039 & M2 & 68 & 0.709560 & 18.7 & Anglo-Saxon & Ringlemere & \\
\hline RING040 & M2 & 78 & 0.709330 & 18.1 & Anglo-Saxon & Ringlemere & \\
\hline RING041 & M2 & 105 & 0.708888 & 18.2 & Anglo-Saxon & Ringlemere & \\
\hline SoU $1355-445 \mathrm{e}$ & M2 & 131 & 0.710661 & 18.2 & Medieval & Southampton Friary & \\
\hline sou $1355-448 \mathrm{e}$ & M2 & 137 & 0.709342 & 18.1 & Medieval & Southampton Friary & \\
\hline SPR-1 & pM2 & 106 & 0.709897 & 19.1 & Roman & Spitalfields & \\
\hline Bees $10 \mathrm{e}$ & no record & 82 & 0.709815 & & Medieval & St Bees & \\
\hline PH 81 SK 4 & M2 & 109 & 0.711430 & 17.5 & Medieval & St Giles & \\
\hline PH 81 SK 10 & M2 & 173 & 0.709360 & 18.4 & Medieval & St Giles & \\
\hline PH 81 SK 11 & M2 & 347 & 0.709760 & 17.1 & Medieval & St Giles & \\
\hline PH 81 SK 16 & M2 & 133 & 0.709580 & 18.2 & Medieval & St Giles & \\
\hline PH 81 SK 18 & M2 & 168 & 0.709450 & 19.8 & Medieval & St Giles & \\
\hline PH 81 SK 21 & M2 & 146 & 0.709620 & 18.4 & Medieval & St Giles & \\
\hline PH 81 SK 48 & M2 & 223 & 0.709610 & 17.7 & Medieval & St Giles & \\
\hline SK001 & $\mathrm{M} 2$ & 268 & 0.709513 & 17.9 & Medieval & St Thomas Kirk & Higher rainfall \\
\hline SK002 & M2 & 363 & 0.709437 & 18.3 & Medieval & St Thomas Kirk & Higher rainfall \\
\hline SK004 & M2 & 254 & 0.709303 & 18.6 & Medieval & St Thomas Kirk & Higher rainfall \\
\hline SK005 & $\mathrm{M} 2$ & 373 & 0.709456 & 18.0 & Medieval & St Thomas Kirk & Higher rainfall \\
\hline SK008 & M2 & 283 & 0.709340 & 19.2 & Medieval & St Thomas Kirk & Higher rainfall \\
\hline SK014 & M2 & 264 & 0.709494 & 18.3 & Medieval & St Thomas Kirk & Higher rainfall \\
\hline SK016 & M2 & 264 & 0.709585 & 18.4 & Medieval & St Thomas Kirk & Higher rainfall \\
\hline SK019 & M2 & 381 & 0.709604 & 18.1 & Medieval & St Thomas Kirk & Higher rainfall \\
\hline 4.10 .4 & pM1 & 56 & 0.708370 & 17.0 & Anglo-Saxon & Stonehenge & \\
\hline ET54 & M1 & 68 & 0.714447 & 18.0 & Bronze Age & Toremore & \\
\hline TP15 & $\mathrm{C}$ & 82 & 0.709195 & & Bronze Age & Towthorpe & \\
\hline TP16 & $\mathrm{C}$ & 72 & 0.709227 & 19.0 & Bronze Age & Towthorpe & \\
\hline TP17 & $\mathrm{pM} 2$ & 56 & 0.710458 & & Bronze Age & Towthorpe & \\
\hline TP18 & M2 & & & 18.3 & Bronze Age & Towthorpe & \\
\hline $\mathrm{S} 105-20$ & $\mathrm{M} 2$ & 71 & 0.710758 & 18.6 & Medieval & Towyn Y Capel & Higher rainfall \\
\hline S105-32 & M3 & 95 & 0.710548 & 18.6 & Medieval & Towyn Y Capel & Higher rainfall \\
\hline SF8658-M2 & M2 & 85 & 0.709970 & 18.0 & Roman & Vindolanda & \\
\hline WASP-001e & pM1 & 52 & 0.711775 & 18.0 & Anglo-Saxon & Wasperton & \\
\hline WASP-006e & M2 & 139 & 0.710348 & 17.8 & Anglo-Saxon & Wasperton & \\
\hline WASP-008e & M2 & 124 & 0.710697 & 18.0 & Anglo-Saxon & Wasperton & \\
\hline WASP-024e & $\mathrm{pM} 1$ & 119 & 0.710614 & 18.2 & Anglo-Saxon & Wasperton & \\
\hline WASP-027e & $\mathrm{pM} 2$ & 115 & 0.710310 & 18.2 & Anglo-Saxon & Wasperton & \\
\hline WASP-028e & M1 & 127 & 0.710196 & 17.2 & Anglo-Saxon & Wasperton & \\
\hline WASP-035e & $\mathrm{pM} 2$ & 78 & 0.711775 & 18.2 & Anglo-Saxon & Wasperton & \\
\hline WASP-042e & M2 & 64 & 0.712394 & 17.7 & Anglo-Saxon & Wasperton & \\
\hline WASP-043e & M? & 197 & 0.710464 & 18.0 & Anglo-Saxon & Wasperton & \\
\hline WASP-046e & $\mathrm{pM} 1$ & 75 & 0.709324 & 19.2 & Anglo-Saxon & Wasperton & \\
\hline WASP-048e & $\mathrm{M}$ ? & 75 & 0.710737 & 17.4 & Anglo-Saxon & Wasperton & \\
\hline WASP-055e & $\mathrm{pM} 2$ & 93 & 0.709609 & 17.7 & Anglo-Saxon & Wasperton & \\
\hline WASP-115e & $\mathrm{pM} 2$ & 146 & 0.710346 & 18.3 & Anglo-Saxon & Wasperton & \\
\hline WASP-138e & M3 & 205 & 0.710503 & 17.3 & Anglo-Saxon & Wasperton & \\
\hline WASP-143e & M1 & 132 & 0.710561 & 18.4 & Anglo-Saxon & Wasperton & \\
\hline WASP-153e & $\mathrm{pM} 1$ & 90 & 0.710719 & 18.3 & Anglo-Saxon & Wasperton & \\
\hline WASP-167e & $\mathrm{pM} 2$ & 149 & 0.710475 & 17.5 & Anglo-Saxon & Wasperton & \\
\hline WASP-173e & M2 & 99 & 0.710484 & 17.7 & Anglo-Saxon & Wasperton & \\
\hline WASP-174e & $\mathrm{pM} 2$ & 92 & 0.709650 & 18.7 & Anglo-Saxon & Wasperton & \\
\hline WASP-180e & M2 & 180 & 0.711190 & 18.8 & Anglo-Saxon & Wasperton & \\
\hline WASP-190e & $\mathrm{pM} 1$ & 71 & 0.708822 & 18.7 & Anglo-Saxon & Wasperton & \\
\hline
\end{tabular}




\begin{tabular}{|c|c|c|c|c|c|c|c|}
\hline WASP-191e & pM1 & 69 & 0.708818 & 18.8 & Anglo-Saxon & Wasperton & \\
\hline WASP-193e & $\mathrm{pM} 1$ & 82 & 0.710088 & 18.0 & Anglo-Saxon & Wasperton & \\
\hline WASP-194e & pM1 & 103 & 0.710634 & 18.1 & Anglo-Saxon & Wasperton & \\
\hline WASP-195e & M2 & 76 & 0.710396 & 17.8 & Anglo-Saxon & Wasperton & \\
\hline G100 & M1 & 50 & 0.709002 & 17.8 & Anglo-Saxon & West Heslerton & \\
\hline G101 & M2 & 56 & 0.708757 & 17.9 & Anglo-Saxon & West Heslerton & \\
\hline G102 & pM2 & 74 & 0.710339 & 18.0 & Anglo-Saxon & West Heslerton & \\
\hline G109 & M1 & 42 & 0.709532 & 18.0 & Anglo-Saxon & West Heslerton & \\
\hline G113 & M2 & 100 & 0.708228 & 18.1 & Anglo-Saxon & West Heslerton & \\
\hline G114 & M1 & 70 & 0.709364 & 17.2 & Anglo-Saxon & West Heslerton & \\
\hline G115 & M2 & 65 & 0.708664 & & Anglo-Saxon & West Heslerton & \\
\hline G117 & $\mathrm{M} 2$ & 34 & 0.708480 & 16.5 & Anglo-Saxon & West Heslerton & \\
\hline G122 & M2 & 66 & 0.709767 & 17.8 & Anglo-Saxon & West Heslerton & \\
\hline G132 & M3 & 72 & 0.709132 & 16.9 & Anglo-Saxon & West Heslerton & \\
\hline G133 & $\mathrm{pM} 1$ & 50 & 0.710228 & 16.6 & Anglo-Saxon & West Heslerton & \\
\hline G139 & M2 & 77 & 0.709189 & 17.2 & Anglo-Saxon & West Heslerton & \\
\hline G144 & pM1 & 84 & 0.709064 & 17.2 & Anglo-Saxon & West Heslerton & \\
\hline G145 & $\mathrm{M}$ & 101 & 0.709549 & 18.1 & Anglo-Saxon & West Heslerton & \\
\hline G149 & $\mathrm{pM} 1$ & 58 & 0.710570 & 16.9 & Anglo-Saxon & West Heslerton & \\
\hline G151 & M3 & 79 & 0.708610 & 16.9 & Anglo-Saxon & West Heslerton & \\
\hline G154 & M1 & 79 & 0.708857 & & Anglo-Saxon & West Heslerton & \\
\hline G158 & $\mathrm{C}$ & 67 & 0.709937 & & Anglo-Saxon & West Heslerton & \\
\hline G159 & M2 & 72 & 0.708990 & 16.7 & Anglo-Saxon & West Heslerton & \\
\hline G162 & M1 & 118 & 0.709014 & & Anglo-Saxon & West Heslerton & \\
\hline G164 & $\mathrm{pM} 1$ & 47 & 0.710808 & 17.7 & Anglo-Saxon & West Heslerton & \\
\hline G166 & M2 & 172 & 0.708796 & & Anglo-Saxon & West Heslerton & \\
\hline G169 & $\mathrm{M} 1$ & 70 & 0.709032 & 15.9 & Anglo-Saxon & West Heslerton & \\
\hline G173 & M3 & 111 & 0.710482 & 17.2 & Anglo-Saxon & West Heslerton & \\
\hline G73 & M2 & 62 & 0.709101 & 17.8 & Anglo-Saxon & West Heslerton & \\
\hline G74 & $\mathrm{M} 1$ & 76 & 0.710061 & 17.6 & Anglo-Saxon & West Heslerton & \\
\hline G75 & $\mathrm{M} 2$ & 50 & 0.709865 & 18.2 & Anglo-Saxon & West Heslerton & \\
\hline G78 & $\mathrm{pM} 2$ & 49 & 0.709502 & 19.1 & Anglo-Saxon & West Heslerton & \\
\hline G84 & M3 & 102 & 0.709485 & 18.0 & Anglo-Saxon & West Heslerton & \\
\hline G89 & $\mathrm{pM} 1$ & 54 & 0.709792 & 18.1 & Anglo-Saxon & West Heslerton & \\
\hline G97 & M2 & 48 & 0.709606 & 17.8 & Anglo-Saxon & West Heslerton & \\
\hline G97 & $\mathrm{M} 2$ & 68 & 0.709895 & 17.7 & Anglo-Saxon & West Heslerton & \\
\hline G98 & M2 & 79 & 0.708498 & 17.8 & Anglo-Saxon & West Heslerton & \\
\hline 2BA229 & M1 & 256 & 0.711080 & 17.5 & Bronze Age & West Heslerton & \\
\hline 2BA283 & M3 & 64 & 0.709572 & 17.0 & Bronze Age & West Heslerton & \\
\hline 2BA589 & M3 & 56 & 0.708973 & 17.1 & Bronze Age & West Heslerton & \\
\hline WHIA-1 & pM1 & 20 & 0.708465 & 16.9 & Iron Age & West Heslerton & \\
\hline WHIA-2 & $\mathrm{pM} 1$ & 50 & 0.711006 & 17.8 & Iron Age & West Heslerton & \\
\hline IR266 & pM1 & 34 & 0.708849 & 17.1 & Bronze Age & West Heslerton & Lower rainfall \\
\hline IR271 & pM1 & 47 & 0.709057 & 17.3 & Bronze Age & West Heslerton & Lower rainfall \\
\hline IR304 & $\mathrm{pM} 1$ & 37 & 0.709010 & 17.1 & Bronze Age & West Heslerton & Lower rainfall \\
\hline WEY08 SK3704 & M2 & 70 & 0.711560 & 15.2 & Medieval & Weymouth & \\
\hline WEY08 SK3706 & $\mathrm{M} 2$ & 84 & 0.710320 & 15.9 & Medieval & Weymouth & \\
\hline WEY08 SK3707 & $\mathrm{M} 2$ & 82 & 0.713060 & 15.1 & Medieval & Weymouth & \\
\hline WEY08 SK3710 & M2 & 74 & 0.710600 & 16.6 & Medieval & Weymouth & \\
\hline WEY08 SK3711 & M2 & 95 & 0.713770 & 13.7 & Medieval & Weymouth & \\
\hline WEYO8 SK3720 & M2 & 117 & 0.712940 & 15.6 & Medieval & Weymouth & \\
\hline WEY08 SK3724 & M2 & 58 & 0.720510 & 15.8 & Medieval & Weymouth & \\
\hline WEY08 SK3730 & $\mathrm{M} 2$ & 98 & 0.710130 & 16.1 & Medieval & Weymouth & \\
\hline WEY08 SK3739 & M2 & 61 & 0.710890 & 15.4 & Medieval & Weymouth & \\
\hline WEYO8 SK3744 & M2 & 85 & 0.710720 & 15.8 & Medieval & Weymouth & \\
\hline
\end{tabular}




\begin{tabular}{|c|c|c|c|c|c|c|}
\hline WCL22-M1 & M1 & & & 17.5 & Anglo-Saxon & Whitton \\
\hline WCL22-M2 & M2 & & & 16.8 & Anglo-Saxon & Whitton \\
\hline WCL22-M3 & M3 & & & 16.5 & Anglo-Saxon & Whitton \\
\hline WCL30-M1 & M1 & & & 17.5 & Anglo-Saxon & Whitton \\
\hline WCL30-M2 & M2 & & & 17.6 & Anglo-Saxon & Whitton \\
\hline WCL30-M3 & M3 & & & 17.5 & Anglo-Saxon & Whitton \\
\hline Castle yard 9 & pM2 & 46 & 0.709340 & 18.2 & Roman & York \\
\hline Clifton 9 & $\mathrm{pM} 2$ & 71 & 0.709950 & 17.9 & Roman & York \\
\hline Hospitium 58 & M2 & 77 & 0.708970 & 18.3 & Roman & York \\
\hline Mount vale a & $\mathrm{pM} 2$ & 146 & 0.708710 & 17.5 & Roman & York \\
\hline Mount vale c & $\mathrm{pM} 2$ & 112 & 0.711750 & 18.1 & Roman & York \\
\hline RE02 & $\mathrm{pM} 2$ & 458 & 0.708080 & 18.3 & Roman & York \\
\hline RE10 & $\mathrm{pM} 2$ & 94 & 0.709960 & 18.0 & Roman & York \\
\hline RE10 & M3 & 63 & 0.710670 & 17.5 & Roman & York \\
\hline RE11 & $\mathrm{pM} 2$ & 96 & 0.710150 & 18.6 & Roman & York \\
\hline RE13 & M2 & 57 & 0.710090 & 17.3 & Roman & York \\
\hline RE14 & M3 & 98 & 0.709700 & 17.5 & Roman & York \\
\hline RE16 & M2 & 65 & 0.709990 & 18.8 & Roman & York \\
\hline RE17 & M3 & 68 & 0.710120 & 18.4 & Roman & York \\
\hline RE17 & $\mathrm{pM} 2$ & 101 & 0.709180 & 17.7 & Roman & York \\
\hline RE18 & $\mathrm{pM} 2$ & 71 & 0.709940 & 17.6 & Roman & York \\
\hline RE21 & pM2 & 45 & 0.710310 & 17.3 & Roman & York \\
\hline RE22 & M2 & 93 & 0.709070 & 18.4 & Roman & York \\
\hline RE23 & pM2 & 226 & 0.709310 & 17.5 & Roman & York \\
\hline RE25 & $\mathrm{pM} 2$ & 108 & 0.709800 & 19.4 & Roman & York \\
\hline RE26 & $\mathrm{pM} 2$ & 87 & 0.709140 & 18.3 & Roman & York \\
\hline RE27 & $\mathrm{pM} 2$ & 174 & 0.708240 & 18.0 & Roman & York \\
\hline RE28 & M1 & 74 & 0.708410 & 18.2 & Roman & York \\
\hline RE3 & M2 & 107 & 0.709390 & 16.9 & Roman & York \\
\hline RE31 & $\mathrm{pM} 2$ & 52 & 0.710450 & 18.9 & Roman & York \\
\hline RE31 & M2 & 52 & 0.710440 & 18.6 & Roman & York \\
\hline RE33 & M3 & 105 & 0.709960 & 18.1 & Roman & York \\
\hline RE36 & M1 & 76 & 0.709360 & 18.6 & Roman & York \\
\hline RE37 & M1 & 42 & 0.710600 & 16.7 & Roman & York \\
\hline RE37 & M2 & 55 & 0.710380 & 16.4 & Roman & York \\
\hline RE4 & $\mathrm{pM} 2$ & 161 & 0.709670 & 18.4 & Roman & York \\
\hline RE41 & M2 & 112 & 0.709520 & 17.4 & Roman & York \\
\hline RE43 & M2 & 65 & 0.708330 & 17.4 & Roman & York \\
\hline RE45 & $\mathrm{pM} 2$ & 117 & 0.709320 & 18.4 & Roman & York \\
\hline RE46 & M2 & 190 & 0.708780 & 17.1 & Roman & York \\
\hline RE47 & $\mathrm{pM} 2$ & 79 & 0.709240 & 18.4 & Roman & York \\
\hline RE48 & M2 & 88 & 0.711170 & 18.2 & Roman & York \\
\hline RE51 & $\mathrm{pM} 2$ & 117 & 0.709060 & 18.9 & Roman & York \\
\hline RE7 & $\mathrm{pM} 2$ & 120 & 0.710070 & 18.1 & Roman & York \\
\hline TDC04 & M2 & 49 & 0.710410 & 16.9 & Roman & York \\
\hline TDC153 & $\mathrm{pM} 2$ & 93 & 0.709550 & 18.4 & Roman & York \\
\hline TDC157 & $\mathrm{pM} 2$ & 162 & 0.708740 & 17.1 & Roman & York \\
\hline TDC173 & M2 & 338 & 0.708480 & 17.5 & Roman & York \\
\hline TDC288 & M3 & 170 & 0.708320 & 17.9 & Roman & York \\
\hline TDC314 & M2 & 56 & 0.709270 & 17.2 & Roman & York \\
\hline TDC411 & $\mathrm{pM} 2$ & 51 & 0.713120 & 17.6 & Roman & York \\
\hline TDC466 & M2 & 146 & 0.710000 & 17.8 & Roman & York \\
\hline TDC513 & M2 & 160 & 0.710180 & 18.6 & Roman & York \\
\hline TDC516 & $\mathrm{pM} 2$ & 284 & 0.708960 & 19.5 & Roman & York \\
\hline TDC608 & M2 & 165 & 0.712930 & 18.3 & Roman & York \\
\hline
\end{tabular}




\begin{tabular}{|l|l|c|c|c|l|l|l|}
\hline TDC708 & $\mathrm{pM} 2$ & 92 & 0.710660 & 18.0 & Roman & York & \\
\hline TDC710 & $\mathrm{pM} 2$ & 103 & 0.713550 & 19.7 & Roman & York & \\
\hline TDCR38 & $\mathrm{M} 2$ & 103 & 0.713080 & 17.4 & Roman & York & \\
\hline The Mount & $\mathrm{pM} 2$ & 175 & 0.709310 & 17.8 & Roman & York & \\
\hline
\end{tabular}




\begin{tabular}{|c|c|c|}
\hline $\begin{array}{l}\text { sample } \\
\text { code }\end{array}$ & $\begin{array}{l}\text { source of } \\
\text { water }\end{array}$ & d180(VSMOW) \\
\hline ECM-001 & shallow well & -5.6 \\
\hline ECM-002 & shallow well & -5.5 \\
\hline ECM-003 & borehole & -5.3 \\
\hline ECM-004 & spring & -5.1 \\
\hline ECM-005 & shallow well & -5.3 \\
\hline ECM-006 & borehole & -3.3 \\
\hline ECM-007 & borehole & -5.2 \\
\hline ECM-008 & borehole & -6.1 \\
\hline ECM-009 & borehole & -4.8 \\
\hline ECM-010 & borehole & -4.7 \\
\hline ECM-011 & borehole & -4.8 \\
\hline ECM-012 & lake & -2.4 \\
\hline ECM-013 & spring & -5.6 \\
\hline ECM-014 & borehole & -7.8 \\
\hline ECM-015 & borehole & -7.6 \\
\hline ECM-016 & borehole & -7.5 \\
\hline ECM-017 & borehole & -7.5 \\
\hline ECM-018 & borehole & -8 \\
\hline ECM-019 & borehole & -7.6 \\
\hline ECM-020 & borehole & -2.5 \\
\hline ECM-021 & borehole & -7.2 \\
\hline ECM-022 & borehole & -7.3 \\
\hline ECM-023 & borehole & -7.2 \\
\hline ECM-024 & borehole & -7.2 \\
\hline ECM-025 & borehole & -7.8 \\
\hline ECM-026 & borehole & -7.5 \\
\hline ECM-027 & borehole & -7.9 \\
\hline ECM-028 & borehole & -7.1 \\
\hline ECM-029 & borehole & -7.1 \\
\hline ECM-030 & borehole & -7.7 \\
\hline ECM-031 & borehole & -7.8 \\
\hline ECM-032 & borehole & -8.1 \\
\hline ECM-033 & borehole & -7.9 \\
\hline ECM-034 & borehole & -7.4 \\
\hline ECM-035 & borehole & -7.8 \\
\hline ECM-036 & borehole & -7.8 \\
\hline ECM-037 & borehole & -8.4 \\
\hline ECM-038 & borehole & -7.9 \\
\hline ECM-039 & borehole & -7.6 \\
\hline ECM-040 & borehole & -8.4 \\
\hline ECM-041 & borehole & -8.5 \\
\hline ECM-042 & borehole & -7.8 \\
\hline ECM-043 & borehole & -8.1 \\
\hline
\end{tabular}




\begin{tabular}{|c|c|c|}
\hline ECM-044 & borehole & -7.8 \\
\hline ECM-045 & borehole & -8.2 \\
\hline ECM-046 & borehole & -8.5 \\
\hline ECM-047 & borehole & -7.5 \\
\hline ECM-048 & borehole & -8.4 \\
\hline ECM-049 & borehole & -8.1 \\
\hline ECM-050 & borehole & -7.3 \\
\hline ECM-051 & borehole & -7.8 \\
\hline ECM-052 & borehole & -7.9 \\
\hline ECM-053 & borehole & -8.4 \\
\hline ECM-054 & borehole & -7.6 \\
\hline ECM-055 & borehole & -8 \\
\hline ECM-056 & borehole & -8.6 \\
\hline ECM-057 & borehole & -8.2 \\
\hline ECM-058 & borehole & -7.7 \\
\hline ECM-059 & borehole & -7.7 \\
\hline ECM-060 & borehole & -8.1 \\
\hline ECM-061 & borehole & -7.8 \\
\hline ECM-062 & borehole & -7.7 \\
\hline ECM-063 & borehole & -8 \\
\hline ECM-064 & borehole & -8.1 \\
\hline ECM-065 & borehole & -8 \\
\hline ECM-066 & borehole & -7.9 \\
\hline ECM-067 & $\begin{array}{l}\text { pumped } \\
\text { sample }\end{array}$ & -6.6 \\
\hline ECM-068 & $\begin{array}{l}\text { pumped } \\
\text { sample }\end{array}$ & -6.6 \\
\hline ECM-069 & $\begin{array}{l}\text { pumped } \\
\text { sample }\end{array}$ & -6.6 \\
\hline ECM-070 & $\begin{array}{l}\text { pumped } \\
\text { sample }\end{array}$ & -6.6 \\
\hline ECM-071 & $\begin{array}{l}\text { pumped } \\
\text { sample }\end{array}$ & -6.5 \\
\hline ECM-072 & $\begin{array}{l}\text { pumped } \\
\text { sample }\end{array}$ & -6.5 \\
\hline ECM-073 & $\begin{array}{l}\text { pumped } \\
\text { sample }\end{array}$ & -5.9 \\
\hline ECM-074 & $\begin{array}{l}\text { pumped } \\
\text { sample }\end{array}$ & -5.9 \\
\hline ECM-075 & $\begin{array}{l}\text { pumped } \\
\text { sample }\end{array}$ & -6.1 \\
\hline ECM-076 & $\begin{array}{l}\text { pumped } \\
\text { sample }\end{array}$ & -6.1 \\
\hline ECM-077 & $\begin{array}{l}\text { pumped } \\
\text { sample }\end{array}$ & -6 \\
\hline ECM-078 & $\begin{array}{l}\text { pumped } \\
\text { sample }\end{array}$ & -6 \\
\hline ECM-079 & $\begin{array}{l}\text { pumped } \\
\text { sample }\end{array}$ & -6.1 \\
\hline ECM-080 & $\begin{array}{l}\text { pumped } \\
\text { sample }\end{array}$ & -6.1 \\
\hline ECM-081 & $\begin{array}{l}\text { pumped } \\
\text { sample }\end{array}$ & -6.8 \\
\hline ECM-082 & $\begin{array}{l}\text { pumped } \\
\text { sample }\end{array}$ & -6.8 \\
\hline ECM-083 & $\begin{array}{l}\text { pumped } \\
\text { sample }\end{array}$ & -6.3 \\
\hline ECM-084 & $\begin{array}{l}\text { pumped } \\
\text { sample }\end{array}$ & -6.3 \\
\hline
\end{tabular}




\begin{tabular}{|c|c|c|}
\hline ECM-085 & $\begin{array}{l}\text { pumped } \\
\text { sample }\end{array}$ & -6.4 \\
\hline ECM-086 & $\begin{array}{l}\text { pumped } \\
\text { sample }\end{array}$ & -6.4 \\
\hline ECM-087 & $\begin{array}{l}\text { pumped } \\
\text { sample }\end{array}$ & -6.2 \\
\hline ECM-088 & $\begin{array}{l}\text { pumped } \\
\text { sample }\end{array}$ & -6.2 \\
\hline ECM-089 & $\begin{array}{l}\text { pumped } \\
\text { sample }\end{array}$ & -6.4 \\
\hline ECM-090 & $\begin{array}{l}\text { pumped } \\
\text { sample }\end{array}$ & -6.2 \\
\hline ECM-091 & $\begin{array}{l}\text { pumped } \\
\text { sample }\end{array}$ & -6.5 \\
\hline ECM-092 & borehole & -8.2 \\
\hline ECM-093 & borehole & -7.5 \\
\hline ECM-094 & borehole & -7.5 \\
\hline ECM-095 & borehole & -8.1 \\
\hline ECM-096 & borehole & -7.5 \\
\hline ECM-097 & borehole & -8.6 \\
\hline ECM-098 & borehole & -8.4 \\
\hline ECM-099 & borehole & -7.6 \\
\hline ECM-100 & borehole & -7.4 \\
\hline ECM-101 & borehole & -8.1 \\
\hline ECM-102 & borehole & -7.7 \\
\hline ECM-103 & borehole & -7.1 \\
\hline ECM-104 & borehole & -7.5 \\
\hline ECM-105 & borehole & -6.5 \\
\hline ECM-106 & borehole & -7.7 \\
\hline ECM-107 & borehole & -8 \\
\hline ECM-108 & borehole & -7.8 \\
\hline ECM-109 & borehole & -8.2 \\
\hline ECM-110 & borehole & -8.2 \\
\hline ECM-111 & borehole & -8 \\
\hline ECM-112 & borehole & -8.1 \\
\hline ECM-113 & borehole & -8.6 \\
\hline ECM-114 & borehole & -8.2 \\
\hline ECM-115 & $\begin{array}{l}\text { pumped } \\
\text { sample }\end{array}$ & -5.8 \\
\hline ECM-116 & $\begin{array}{l}\text { pumped } \\
\text { sample }\end{array}$ & -6.7 \\
\hline ECM-117 & $\begin{array}{l}\text { pumped } \\
\text { sample }\end{array}$ & -6.3 \\
\hline ECM-118 & $\begin{array}{l}\text { pumped } \\
\text { sample }\end{array}$ & -6.4 \\
\hline ECM-119 & $\begin{array}{l}\text { pumped } \\
\text { sample }\end{array}$ & -6.7 \\
\hline ECM-120 & borehole & -7.4 \\
\hline ECM-121 & borehole & -7.2 \\
\hline ECM-122 & borehole & -7.5 \\
\hline ECM-123 & borehole & -7.9 \\
\hline ECM-124 & borehole & -7.6 \\
\hline ECM-125 & borehole & -8 \\
\hline ECM-126 & borehole & -7.6 \\
\hline
\end{tabular}




\begin{tabular}{|c|c|c|}
\hline ECM-127 & borehole & -7.7 \\
\hline ECM-128 & spring & -7.8 \\
\hline ECM-129 & borehole & -8.1 \\
\hline ECM-130 & borehole & -7.6 \\
\hline ECM-131 & borehole & -8.1 \\
\hline ECM-132 & borehole & -8 \\
\hline ECM-133 & borehole & -8 \\
\hline ECM-134 & borehole & -8.1 \\
\hline ECM-135 & borehole & -8.1 \\
\hline ECM-136 & borehole & -7.8 \\
\hline ECM-137 & borehole & -7.9 \\
\hline ECM-138 & borehole & -8.3 \\
\hline ECM-139 & shallow well & -7.6 \\
\hline ECM-140 & borehole & -7.4 \\
\hline ECM-141 & borehole & -8.1 \\
\hline ECM-142 & borehole & -7.3 \\
\hline ECM-143 & borehole & -7.2 \\
\hline ECM-144 & borehole & -7.4 \\
\hline ECM-145 & borehole & -7.3 \\
\hline ECM-146 & borehole & -7.1 \\
\hline ECM-147 & borehole & -7.2 \\
\hline ECM-148 & borehole & -7.4 \\
\hline ECM-149 & stream & -6.9 \\
\hline ECM-150 & borehole & -7.2 \\
\hline ECM-151 & borehole & -7.6 \\
\hline ECM-152 & borehole & -7.5 \\
\hline ECM-153 & borehole & -7.1 \\
\hline ECM-154 & borehole & -7.07 \\
\hline ECM-155 & borehole & -7.17 \\
\hline ECM-156 & spring & -7.08 \\
\hline ECM-157 & borehole & -6.91 \\
\hline ECM-158 & stream & -7.26 \\
\hline ECM-159 & borehole & -7.16 \\
\hline ECM-160 & borehole & -7.27 \\
\hline ECM-161 & borehole & -7.47 \\
\hline ECM-162 & borehole & -7.15 \\
\hline ECM-163 & borehole & -7.5 \\
\hline ECM-164 & borehole & -7.01 \\
\hline ECM-165 & borehole & -7.1 \\
\hline ECM-166 & borehole & -7.05 \\
\hline ECM-167 & borehole & -7.15 \\
\hline ECM-168 & spring & -7.18 \\
\hline ECM-169 & borehole & -7 \\
\hline ECM-170 & borehole & -7.2 \\
\hline ECM-171 & borehole & -6.99 \\
\hline
\end{tabular}




\begin{tabular}{|c|c|c|}
\hline ECM-172 & spring & -7.08 \\
\hline ECM-173 & borehole & -7.01 \\
\hline ECM-174 & borehole & -6.89 \\
\hline ECM-175 & borehole & -6.72 \\
\hline ECM-176 & river & -6.21 \\
\hline ECM-177 & stream & -6.52 \\
\hline ECM-178 & borehole & -6.95 \\
\hline ECM-179 & borehole & -7.47 \\
\hline ECM-180 & borehole & -7.42 \\
\hline ECM-181 & borehole & -7.5 \\
\hline ECM-182 & stream & -6.65 \\
\hline ECM-183 & stream & -6.62 \\
\hline ECM-184 & stream & -6.81 \\
\hline ECM-185 & stream & -6.24 \\
\hline ECM-186 & borehole & -7.32 \\
\hline ECM-187 & borehole & -6.787 \\
\hline ECM-188 & borehole & -6.98 \\
\hline ECM-189 & borehole & -7.09 \\
\hline ECM-190 & borehole & -7.36 \\
\hline ECM-191 & borehole & -7.3 \\
\hline ECM-192 & borehole & -6.58 \\
\hline ECM-193 & borehole & -6.58 \\
\hline ECM-194 & borehole & -8.18 \\
\hline ECM-195 & stream & -7.01 \\
\hline ECM-196 & river & -6.99 \\
\hline ECM-197 & spring & -7.67 \\
\hline ECM-198 & spring & -7.7 \\
\hline ECM-199 & spring & -7.12 \\
\hline ECM-200 & borehole & -7.39 \\
\hline ECM-201 & borehole & -7.23 \\
\hline ECM-202 & borehole & -7.49 \\
\hline ECM-203 & borehole & -7.16 \\
\hline ECM-204 & borehole & -7.71 \\
\hline ECM-205 & borehole & -7.14 \\
\hline ECM-206 & borehole & -7.08 \\
\hline ECM-207 & borehole & -8.18 \\
\hline ECM-208 & stream & -6.59 \\
\hline ECM-209 & river & -7.11 \\
\hline ECM-210 & spring & -6.97 \\
\hline ECM-211 & $\begin{array}{l}\text { pumped } \\
\text { sample }\end{array}$ & -7.38 \\
\hline ECM-212 & $\begin{array}{l}\text { pumped } \\
\text { sample }\end{array}$ & -6.48 \\
\hline ECM-213 & $\begin{array}{l}\text { pumped } \\
\text { sample }\end{array}$ & -7.15 \\
\hline ECM-214 & borehole & -7.14 \\
\hline ECM-215 & borehole & -6.83 \\
\hline
\end{tabular}




\begin{tabular}{|c|c|c|}
\hline ECM-216 & stream & -7.05 \\
\hline ECM-217 & borehole & -6.27 \\
\hline ECM-218 & borehole & -6.67 \\
\hline ECM-219 & spring & -6.97 \\
\hline ECM-220 & borehole & -7.56 \\
\hline ECM-221 & stream & -6.22 \\
\hline ECM-222 & stream & -6.68 \\
\hline ECM-223 & river & -7.01 \\
\hline ECM-224 & borehole & -5.78 \\
\hline ECM-225 & borehole & -5.5 \\
\hline ECM-226 & borehole & -6.1 \\
\hline ECM-227 & borehole & -7.28 \\
\hline ECM-228 & borehole & -6.93 \\
\hline ECM-229 & borehole & -7.02 \\
\hline ECM-230 & spring & -7.09 \\
\hline ECM-231 & borehole & -7.38 \\
\hline ECM-232 & stream & -6.02 \\
\hline ECM-233 & stream & -6.37 \\
\hline ECM-234 & river & -6.9 \\
\hline ECM-235 & borehole & -7.17 \\
\hline ECM-236 & borehole & -6.87 \\
\hline ECM-237 & borehole & -7.1 \\
\hline ECM-238 & borehole & -7.56 \\
\hline ECM-239 & borehole & -6.73 \\
\hline ECM-240 & borehole & -7.22 \\
\hline ECM-241 & borehole & -8.14 \\
\hline ECM-242 & borehole & -7.87 \\
\hline ECM-243 & borehole & -6.46 \\
\hline ECM-244 & borehole & -6.9 \\
\hline ECM-245 & borehole & -6.97 \\
\hline ECM-246 & spring & -7 \\
\hline ECM-247 & river & -6.47 \\
\hline ECM-248 & borehole & -6.61 \\
\hline ECM-249 & borehole & -7.25 \\
\hline ECM-250 & borehole & -7.4 \\
\hline ECM-251 & $\begin{array}{l}\text { pumped } \\
\text { sample }\end{array}$ & -7.34 \\
\hline ECM-252 & $\begin{array}{l}\text { pumped } \\
\text { sample }\end{array}$ & -7.13 \\
\hline ECM-253 & $\begin{array}{l}\text { pumped } \\
\text { sample }\end{array}$ & -6.28 \\
\hline ECM-254 & $\begin{array}{l}\text { pumped } \\
\text { sample }\end{array}$ & -7.25 \\
\hline ECM-255 & $\begin{array}{l}\text { pumped } \\
\text { sample }\end{array}$ & -7.05 \\
\hline ECM-256 & $\begin{array}{l}\text { pumped } \\
\text { sample }\end{array}$ & -7.04 \\
\hline ECM-257 & $\begin{array}{l}\text { pumped } \\
\text { sample }\end{array}$ & -6.95 \\
\hline ECM-258 & $\begin{array}{l}\text { pumped } \\
\text { sample }\end{array}$ & -7.46 \\
\hline
\end{tabular}




\begin{tabular}{|c|c|c|}
\hline ECM-259 & $\begin{array}{l}\text { pumped } \\
\text { sample }\end{array}$ & -6.98 \\
\hline ECM-260 & $\begin{array}{l}\text { pumped } \\
\text { sample }\end{array}$ & -7.45 \\
\hline ECM-261 & $\begin{array}{l}\text { pumped } \\
\text { sample }\end{array}$ & -7.35 \\
\hline ECM-262 & $\begin{array}{l}\text { pumped } \\
\text { sample }\end{array}$ & -7.57 \\
\hline ECM-263 & borehole & -7.7 \\
\hline ECM-264 & borehole & -7.71 \\
\hline ECM-265 & borehole & -7.99 \\
\hline ECM-266 & borehole & -7.91 \\
\hline ECM-267 & borehole & -7.33 \\
\hline ECM-268 & borehole & -7.19 \\
\hline ECM-269 & borehole & -7.08 \\
\hline ECM-270 & borehole & -7.37 \\
\hline ECM-271 & borehole & -7.23 \\
\hline ECM-272 & borehole & -7.71 \\
\hline ECM-273 & borehole & -7.74 \\
\hline ECM-274 & borehole & -7.7 \\
\hline ECM-275 & borehole & -7.56 \\
\hline ECM-276 & borehole & -7.54 \\
\hline ECM-277 & borehole & -7.43 \\
\hline ECM-278 & borehole & -7.2 \\
\hline ECM-279 & borehole & -7.64 \\
\hline ECM-280 & borehole & -7.72 \\
\hline ECM-281 & borehole & -7.68 \\
\hline ECM-282 & borehole & -7.71 \\
\hline ECM-283 & borehole & -7.95 \\
\hline ECM-284 & borehole & -7.41 \\
\hline ECM-285 & borehole & -7.56 \\
\hline ECM-286 & borehole & -7.58 \\
\hline ECM-287 & borehole & -7.36 \\
\hline ECM-288 & borehole & -7.56 \\
\hline ECM-289 & borehole & -7.57 \\
\hline ECM-290 & spring & -7.17 \\
\hline ECM-291 & borehole & -7.78 \\
\hline ECM-292 & borehole & -7.31 \\
\hline ECM-293 & borehole & -7.66 \\
\hline ECM-294 & spring & -7.3 \\
\hline ECM-295 & borehole & -7.56 \\
\hline ECM-296 & borehole & -7.57 \\
\hline ECM-297 & borehole & -7.21 \\
\hline ECM-298 & borehole & -7.38 \\
\hline ECM-299 & spring & -7.14 \\
\hline ECM-300 & borehole & -7.23 \\
\hline ECM-301 & borehole & -7.37 \\
\hline ECM-302 & river & -7.03 \\
\hline
\end{tabular}




\begin{tabular}{|c|c|c|}
\hline ECM-303 & borehole & -7.06 \\
\hline ECM-304 & borehole & -7.13 \\
\hline ECM-305 & borehole & -6.84 \\
\hline ECM-306 & borehole & -8.8 \\
\hline ECM-307 & borehole & -7.6 \\
\hline ECM-308 & river & -7.01 \\
\hline ECM-309 & river & -6.53 \\
\hline ECM-310 & river & -5.96 \\
\hline ECM-311 & river & -6.56 \\
\hline ECM-312 & river & -6.15 \\
\hline ECM-313 & river & -6.4 \\
\hline ECM-314 & river & -6.44 \\
\hline ECM-315 & borehole & -7.36 \\
\hline ECM-316 & borehole & -7.24 \\
\hline ECM-317 & borehole & -7.21 \\
\hline ECM-318 & borehole & -7.3 \\
\hline ECM-319 & borehole & -8.06 \\
\hline ECM-320 & borehole & -8.36 \\
\hline ECM-321 & borehole & -8.39 \\
\hline ECM-322 & borehole & -8.4 \\
\hline ECM-323 & borehole & -8.34 \\
\hline ECM-324 & borehole & -8.13 \\
\hline ECM-325 & borehole & -8.4 \\
\hline ECM-326 & borehole & -8.2 \\
\hline ECM-327 & borehole & -8.06 \\
\hline ECM-328 & borehole & -8.36 \\
\hline ECM-329 & spring & -7.801 \\
\hline ECM-330 & borehole & -8.02 \\
\hline ECM-331 & borehole & -8.14 \\
\hline ECM-332 & borehole & -8.42 \\
\hline ECM-333 & borehole & -7.79 \\
\hline ECM-334 & borehole & -8.38 \\
\hline ECM-335 & borehole & -6.8 \\
\hline ECM-336 & spring & -7.04 \\
\hline ECM-337 & borehole & -6.79 \\
\hline ECM-338 & borehole & -6.73 \\
\hline ECM-339 & spring & -6.16 \\
\hline ECM-340 & spring & -6.5 \\
\hline ECM-341 & shallow well & -6.4 \\
\hline ECM-342 & borehole & -7.03 \\
\hline ECM-343 & borehole & -6.7 \\
\hline ECM-344 & spring & -6.09 \\
\hline ECM-345 & spring & -6.18 \\
\hline ECM-346 & borehole & -7.29 \\
\hline ECM-347 & borehole & -7.31 \\
\hline
\end{tabular}




\begin{tabular}{|c|c|c|}
\hline ECM-348 & borehole & -7.41 \\
\hline ECM-349 & borehole & -7.59 \\
\hline ECM-350 & borehole & -7.68 \\
\hline ECM-351 & borehole & -7.51 \\
\hline ECM-352 & borehole & -7.75 \\
\hline ECM-353 & borehole & -7.27 \\
\hline ECM-354 & borehole & -7.64 \\
\hline ECM-355 & borehole & -7.51 \\
\hline ECM-356 & borehole & -7.11 \\
\hline ECM-357 & borehole & -7.47 \\
\hline ECM-358 & borehole & -7.47 \\
\hline ECM-359 & borehole & -7.12 \\
\hline ECM-360 & borehole & -6.84 \\
\hline ECM-361 & borehole & -6.9 \\
\hline ECM-362 & borehole & -7.09 \\
\hline ECM-363 & borehole & -7.35 \\
\hline ECM-364 & borehole & -7.23 \\
\hline ECM-365 & borehole & -7 \\
\hline ECM-366 & river & -6.46 \\
\hline ECM-367 & river & -7.15 \\
\hline ECM-368 & river & -6.57 \\
\hline ECM-369 & river & -7.37 \\
\hline ECM-370 & borehole & -7.23 \\
\hline ECM-371 & borehole & -6.88 \\
\hline ECM-372 & borehole & -7.43 \\
\hline ECM-373 & borehole & -7.02 \\
\hline ECM-374 & borehole & -7.69 \\
\hline ECM-375 & borehole & -7.34 \\
\hline ECM-376 & borehole & -6.54 \\
\hline ECM-377 & borehole & -6.97 \\
\hline ECM-378 & borehole & -7.05 \\
\hline ECM-379 & borehole & -7.09 \\
\hline ECM-380 & borehole & -7.01 \\
\hline ECM-381 & borehole & -7.4 \\
\hline ECM-382 & $\begin{array}{l}\text { pumped } \\
\text { sample }\end{array}$ & -7.18 \\
\hline ECM-383 & $\begin{array}{l}\text { pumped } \\
\text { sample }\end{array}$ & -6.79 \\
\hline ECM-384 & $\begin{array}{l}\text { pumped } \\
\text { sample }\end{array}$ & -6.85 \\
\hline ECM-385 & $\begin{array}{l}\text { pumped } \\
\text { sample }\end{array}$ & -7.25 \\
\hline ECM-386 & $\begin{array}{l}\text { pumped } \\
\text { sample }\end{array}$ & -7.27 \\
\hline ECM-387 & shallow well & -6.96 \\
\hline ECM-388 & shallow well & -7.07 \\
\hline ECM-389 & $\begin{array}{l}\text { pumped } \\
\text { sample }\end{array}$ & -7.05 \\
\hline ECM-390 & $\begin{array}{l}\text { pumped } \\
\text { sample }\end{array}$ & -7.68 \\
\hline
\end{tabular}




\begin{tabular}{|c|c|c|}
\hline ECM-391 & river & -6.98 \\
\hline ECM-392 & river & -7.09 \\
\hline ECM-393 & river & -7.14 \\
\hline ECM-394 & river & -7.22 \\
\hline ECM-395 & river & -7.04 \\
\hline ECM-396 & river & -6.96 \\
\hline ECM-397 & river & -7.21 \\
\hline ECM-398 & river & -7.11 \\
\hline ECM-399 & borehole & -7.31 \\
\hline ECM-400 & borehole & -7.12 \\
\hline ECM-401 & borehole & -7.07 \\
\hline ECM-402 & borehole & -7.32 \\
\hline ECM-403 & borehole & -7.27 \\
\hline ECM-404 & borehole & -6.7 \\
\hline ECM-405 & borehole & -6.67 \\
\hline ECM-406 & borehole & -6.88 \\
\hline ECM-407 & borehole & -6.85 \\
\hline ECM-408 & borehole & -6.92 \\
\hline ECM-409 & artesian & -6.41 \\
\hline ECM-410 & borehole & -6.63 \\
\hline ECM-411 & borehole & -6.92 \\
\hline ECM-412 & stream & -6.85 \\
\hline ECM-413 & borehole & -6.94 \\
\hline ECM-414 & borehole & -7.38 \\
\hline ECM-415 & borehole & -7.2 \\
\hline ECM-416 & borehole & -7.27 \\
\hline ECM-417 & borehole & -7.68 \\
\hline ECM-418 & borehole & -7.98 \\
\hline ECM-419 & borehole & -7.48 \\
\hline ECM-420 & borehole & -7.28 \\
\hline ECM-421 & borehole & -7.39 \\
\hline ECM-422 & borehole & -7.14 \\
\hline ECM-423 & borehole & -7.52 \\
\hline ECM-424 & borehole & -7.73 \\
\hline ECM-425 & borehole & -7.05 \\
\hline ECM-426 & borehole & -7.111 \\
\hline ECM-427 & borehole & -7.66 \\
\hline ECM-428 & borehole & -7.23 \\
\hline ECM-429 & borehole & -7.42 \\
\hline ECM-430 & borehole & -7.42 \\
\hline ECM-431 & borehole & -7.14 \\
\hline ECM-432 & borehole & -7.07 \\
\hline ECM-433 & borehole & -7.48 \\
\hline ECM-434 & borehole & -7.48 \\
\hline ECM-435 & borehole & -7.92 \\
\hline
\end{tabular}




\begin{tabular}{|c|c|c|}
\hline ECM-436 & borehole & -8.11 \\
\hline ECM-437 & borehole & -7.03 \\
\hline ECM-438 & borehole & -7.95 \\
\hline ECM-439 & borehole & -8.82 \\
\hline ECM-440 & borehole & -7.79 \\
\hline ECM-441 & borehole & -7.17 \\
\hline ECM-442 & borehole & -8.05 \\
\hline ECM-443 & borehole & -7.44 \\
\hline ECM-444 & spring & -7.02 \\
\hline ECM-445 & borehole & -7.21 \\
\hline ECM-446 & spring & -7.18 \\
\hline ECM-447 & borehole & -7.32 \\
\hline ECM-448 & spring & -7.1 \\
\hline ECM-449 & borehole & -7.11 \\
\hline ECM-450 & borehole & -5.87 \\
\hline ECM-451 & borehole & -7.52 \\
\hline ECM-452 & borehole & -7.48 \\
\hline ECM-453 & borehole & -8.15 \\
\hline ECM-454 & borehole & -8.07 \\
\hline ECM-455 & $\begin{array}{l}\text { pumped } \\
\text { sample }\end{array}$ & -7.55 \\
\hline ECM-456 & $\begin{array}{l}\text { pumped } \\
\text { sample }\end{array}$ & -7.44 \\
\hline ECM-457 & $\begin{array}{l}\text { pumped } \\
\text { sample }\end{array}$ & -7.91 \\
\hline ECM-458 & $\begin{array}{l}\text { pumped } \\
\text { sample }\end{array}$ & -6.44 \\
\hline ECM-459 & $\begin{array}{l}\text { pumped } \\
\text { sample }\end{array}$ & -7.51 \\
\hline ECM-460 & spring & -6.27 \\
\hline ECM-461 & spring & -4.92 \\
\hline ECM-462 & borehole & -4.99 \\
\hline ECM-463 & borehole & -5.23 \\
\hline ECM-464 & spring & -5.29 \\
\hline ECM-465 & borehole & -5.42 \\
\hline ECM-466 & shallow well & -4.94 \\
\hline ECM-467 & spring & -4.87 \\
\hline ECM-468 & borehole & -4.86 \\
\hline ECM-469 & spring & -4.8 \\
\hline ECM-470 & borehole & -4.81 \\
\hline ECM-471 & spring & -5.97 \\
\hline ECM-472 & borehole & -5.63 \\
\hline ECM-473 & borehole & -6.28 \\
\hline ECM-474 & borehole & -5.71 \\
\hline ECM-475 & borehole & -7.16 \\
\hline ECM-476 & spring & -7.22 \\
\hline ECM-477 & spring & -7.1 \\
\hline ECM-478 & borehole & -7.26 \\
\hline
\end{tabular}




\begin{tabular}{|c|c|c|}
\hline ECM-479 & borehole & -6.54 \\
\hline ECM-480 & borehole & -6.31 \\
\hline ECM-481 & borehole & -6.63 \\
\hline ECM-482 & borehole & -7.66 \\
\hline ECM-483 & borehole & -7.26 \\
\hline ECM-484 & borehole & -5.82 \\
\hline ECM-485 & borehole & -7.54 \\
\hline ECM-486 & borehole & -7.44 \\
\hline ECM-487 & borehole & -7.45 \\
\hline ECM-488 & borehole & -7.59 \\
\hline ECM-489 & borehole & -7.34 \\
\hline ECM-490 & borehole & -7.29 \\
\hline ECM-491 & borehole & -7.19 \\
\hline ECM-492 & borehole & -7.27 \\
\hline ECM-493 & borehole & -7.48 \\
\hline ECM-494 & borehole & -7.35 \\
\hline ECM-495 & borehole & -7.21 \\
\hline ECM-496 & borehole & -7.24 \\
\hline ECM-497 & borehole & -7.16 \\
\hline ECM-498 & stream & -7.45 \\
\hline ECM-499 & borehole & -7.24 \\
\hline ECM-500 & borehole & -7.57 \\
\hline ECM-501 & borehole & -7.31 \\
\hline ECM-502 & borehole & -7.41 \\
\hline ECM-503 & borehole & -7.38 \\
\hline ECM-504 & borehole & -7.04 \\
\hline ECM-505 & borehole & -7.07 \\
\hline ECM-506 & borehole & -7.3 \\
\hline ECM-507 & borehole & -7.42 \\
\hline ECM-508 & borehole & -7.51 \\
\hline ECM-509 & borehole & -7.56 \\
\hline ECM-510 & borehole & -7.25 \\
\hline ECM-511 & borehole & -7.48 \\
\hline ECM-512 & borehole & -7.31 \\
\hline ECM-513 & borehole & -7.32 \\
\hline ECM-514 & borehole & -7.29 \\
\hline ECM-515 & borehole & -7.75 \\
\hline ECM-516 & borehole & -7.77 \\
\hline ECM-517 & borehole & -7.71 \\
\hline ECM-518 & borehole & -7.65 \\
\hline ECM-519 & borehole & -7.29 \\
\hline ECM-520 & borehole & -7.24 \\
\hline ECM-521 & borehole & -7.14 \\
\hline ECM-522 & borehole & -6.89 \\
\hline ECM-523 & borehole & -7.2 \\
\hline
\end{tabular}




\begin{tabular}{|c|c|c|}
\hline ECM-524 & borehole & -7.28 \\
\hline ECM-525 & shallow well & -6.37 \\
\hline ECM-526 & borehole & -7.69 \\
\hline ECM-527 & borehole & -7.73 \\
\hline ECM-528 & borehole & -7.47 \\
\hline ECM-529 & borehole & -6.7 \\
\hline ECM-530 & borehole & -6.94 \\
\hline ECM-531 & borehole & -7.89 \\
\hline ECM-532 & borehole & -7.55 \\
\hline ECM-533 & borehole & -7.65 \\
\hline ECM-534 & borehole & -7.52 \\
\hline ECM-535 & borehole & -7.6 \\
\hline ECM-536 & spring & -6.74 \\
\hline ECM-537 & spring & -6.5 \\
\hline ECM-538 & spring & -6.38 \\
\hline ECM-539 & borehole & -6.53 \\
\hline ECM-540 & borehole & -6.64 \\
\hline ECM-541 & borehole & -7.69 \\
\hline ECM-542 & borehole & -7.56 \\
\hline ECM-543 & borehole & -7.11 \\
\hline ECM-544 & borehole & -6.8 \\
\hline ECM-545 & borehole & -7.1 \\
\hline ECM-546 & borehole & -6.98 \\
\hline ECM-547 & borehole & -6.6 \\
\hline ECM-548 & borehole & -7.03 \\
\hline ECM-549 & spring & -7.01 \\
\hline ECM-550 & borehole & -7.1 \\
\hline ECM-551 & borehole & -7.25 \\
\hline ECM-552 & spring & -7.59 \\
\hline ECM-553 & borehole & -6.63 \\
\hline ECM-554 & borehole & -7.18 \\
\hline ECM-555 & borehole & -7.17 \\
\hline ECM-556 & borehole & -6.91 \\
\hline ECM-557 & borehole & -6.46 \\
\hline ECM-558 & borehole & -7.29 \\
\hline ECM-559 & borehole & -7.23 \\
\hline ECM-560 & borehole & -7.33 \\
\hline ECM-561 & borehole & -7.37 \\
\hline ECM-562 & borehole & -7.28 \\
\hline ECM-563 & borehole & -7.52 \\
\hline ECM-564 & borehole & -7.5 \\
\hline ECM-565 & borehole & -5.97 \\
\hline ECM-566 & borehole & -6.11 \\
\hline ECM-567 & borehole & -6.74 \\
\hline ECM-568 & stream & -6.19 \\
\hline
\end{tabular}




\begin{tabular}{|c|c|c|}
\hline ECM-569 & stream & -7.24 \\
\hline ECM-570 & borehole & -7.76 \\
\hline ECM-571 & spring & -8.06 \\
\hline ECM-572 & spring & -8.41 \\
\hline ECM-573 & borehole & -7.63 \\
\hline ECM-574 & borehole & -7.54 \\
\hline ECM-575 & borehole & -8.57 \\
\hline ECM-576 & borehole & -8.71 \\
\hline ECM-577 & borehole & -7.13 \\
\hline ECM-578 & borehole & -7.66 \\
\hline ECM-579 & borehole & -8.06 \\
\hline ECM-580 & borehole & -8.78 \\
\hline ECM-581 & borehole & -8.71 \\
\hline ECM-582 & borehole & -8.71 \\
\hline ECM-583 & borehole & -7.77 \\
\hline ECM-584 & borehole & -7.77 \\
\hline ECM-585 & borehole & -8.23 \\
\hline ECM-586 & borehole & -8.225 \\
\hline ECM-587 & borehole & -8.55 \\
\hline ECM-588 & borehole & -8.545 \\
\hline ECM-589 & borehole & -8.94 \\
\hline ECM-590 & borehole & -7.33 \\
\hline ECM-591 & spring & -6.7 \\
\hline ECM-592 & stream & -7.47 \\
\hline ECM-593 & borehole & -7.04 \\
\hline ECM-594 & borehole & -6.87 \\
\hline ECM-595 & borehole & -6.79 \\
\hline ECM-596 & borehole & -7.27 \\
\hline ECM-597 & borehole & -7.47 \\
\hline ECM-598 & borehole & -6.82 \\
\hline ECM-599 & borehole & -7.6 \\
\hline ECM-600 & borehole & -7.53 \\
\hline ECM-601 & borehole & -7.29 \\
\hline ECM-602 & borehole & -7.28 \\
\hline ECM-603 & borehole & -7.44 \\
\hline ECM-604 & borehole & -7.33 \\
\hline ECM-605 & artesian & -8.04 \\
\hline ECM-606 & artesian & -8.01 \\
\hline ECM-607 & artesian & -8.61 \\
\hline ECM-608 & artesian & -8.63 \\
\hline ECM-609 & $\begin{array}{l}\text { pumped } \\
\text { sample }\end{array}$ & -8.36 \\
\hline ECM-610 & $\begin{array}{l}\text { pumped } \\
\text { sample }\end{array}$ & -8.16 \\
\hline ECM-611 & $\begin{array}{l}\text { pumped } \\
\text { sample }\end{array}$ & -7.71 \\
\hline ECM-612 & artesian & -7.92 \\
\hline
\end{tabular}




\begin{tabular}{|c|c|c|}
\hline ECM-613 & $\begin{array}{l}\text { pumped } \\
\text { sample }\end{array}$ & -8.12 \\
\hline ECM-614 & $\begin{array}{l}\text { pumped } \\
\text { sample }\end{array}$ & -7.94 \\
\hline ECM-615 & $\begin{array}{l}\text { pumped } \\
\text { sample }\end{array}$ & -7.71 \\
\hline ECM-616 & artesian & -7.91 \\
\hline ECM-617 & artesian & -8.67 \\
\hline ECM-618 & artesian & -7.87 \\
\hline ECM-619 & artesian & -8.65 \\
\hline ECM-620 & artesian & -8.27 \\
\hline ECM-621 & artesian & -8.91 \\
\hline ECM-622 & $\begin{array}{l}\text { pumped } \\
\text { sample }\end{array}$ & -7.89 \\
\hline ECM-623 & $\begin{array}{l}\text { pumped } \\
\text { sample }\end{array}$ & -8.15 \\
\hline ECM-624 & $\begin{array}{l}\text { pumped } \\
\text { sample }\end{array}$ & -8.05 \\
\hline ECM-625 & $\begin{array}{l}\text { pumped } \\
\text { sample }\end{array}$ & -7.76 \\
\hline ECM-626 & $\begin{array}{l}\text { pumped } \\
\text { sample }\end{array}$ & -7.95 \\
\hline ECM-627 & borehole & -7.4 \\
\hline ECM-628 & borehole & -7.33 \\
\hline ECM-629 & borehole & -7.47 \\
\hline ECM-630 & borehole & -7.56 \\
\hline ECM-631 & borehole & -7.49 \\
\hline ECM-632 & borehole & -7.58 \\
\hline ECM-633 & borehole & -7.3 \\
\hline ECM-634 & stream & -7.22 \\
\hline ECM-635 & spring & -6.85 \\
\hline ECM-636 & spring & -6.41 \\
\hline ECM-637 & stream & -7.21 \\
\hline ECM-638 & spring & -6.28 \\
\hline ECM-639 & stream & -6.15 \\
\hline ECM-640 & spring & -6.17 \\
\hline ECM-641 & shallow well & -6.62 \\
\hline ECM-642 & spring & -5.63 \\
\hline ECM-643 & borehole & -6.41 \\
\hline ECM-644 & shallow well & -7.16 \\
\hline ECM-645 & spring & -6.29 \\
\hline ECM-646 & shallow well & -6.31 \\
\hline ECM-647 & borehole & -6.54 \\
\hline ECM-648 & spring & -6.89 \\
\hline ECM-649 & spring & -6.67 \\
\hline ECM-650 & spring & -6.4 \\
\hline ECM-651 & borehole & -6.24 \\
\hline ECM-652 & borehole & -6.88 \\
\hline ECM-653 & borehole & -6.84 \\
\hline ECM-654 & spring & -6.8 \\
\hline ECM-655 & spring & -6.63 \\
\hline
\end{tabular}




\begin{tabular}{|c|c|c|}
\hline ECM-656 & borehole & -8.06 \\
\hline ECM-657 & borehole & -8.34 \\
\hline ECM-658 & borehole & -8.5 \\
\hline ECM-659 & borehole & -8.21 \\
\hline ECM-660 & borehole & -8.49 \\
\hline ECM-661 & borehole & -8.5 \\
\hline ECM-662 & borehole & -8.66 \\
\hline ECM-663 & borehole & -8.25 \\
\hline ECM-664 & spring & -8 \\
\hline ECM-665 & spring & -7.94 \\
\hline ECM-666 & spring & -8.51 \\
\hline ECM-667 & spring & -8.38 \\
\hline ECM-668 & spring & -7.58 \\
\hline ECM-669 & spring & -7.43 \\
\hline ECM-670 & spring & -7.79 \\
\hline ECM-671 & spring & -7.54 \\
\hline ECM-672 & spring & -7.71 \\
\hline ECM-673 & spring & -8.8 \\
\hline ECM-674 & spring & -8.61 \\
\hline ECM-675 & spring & -8.47 \\
\hline ECM-676 & spring & -9 \\
\hline ECM-677 & spring & -8.73 \\
\hline ECM-678 & spring & -9.04 \\
\hline ECM-679 & borehole & -7.23 \\
\hline ECM-680 & borehole & -7.03 \\
\hline ECM-681 & borehole & -7.33 \\
\hline ECM-682 & borehole & -8.84 \\
\hline ECM-683 & stream & -7.18 \\
\hline ECM-684 & $\begin{array}{l}\text { pumped } \\
\text { sample }\end{array}$ & -6.9 \\
\hline ECM-685 & $\begin{array}{l}\text { pumped } \\
\text { sample }\end{array}$ & -7.33 \\
\hline ECM-686 & $\begin{array}{l}\text { pumped } \\
\text { sample }\end{array}$ & -7.02 \\
\hline ECM-687 & $\begin{array}{l}\text { pumped } \\
\text { sample }\end{array}$ & -6.96 \\
\hline ECM-688 & $\begin{array}{l}\text { pumped } \\
\text { sample }\end{array}$ & -7.21 \\
\hline ECM-689 & $\begin{array}{l}\text { pumped } \\
\text { sample }\end{array}$ & -7.39 \\
\hline ECM-690 & $\begin{array}{l}\text { pumped } \\
\text { sample }\end{array}$ & -7.08 \\
\hline ECM-691 & $\begin{array}{l}\text { pumped } \\
\text { sample }\end{array}$ & -7.3 \\
\hline ECM-692 & $\begin{array}{l}\text { pumped } \\
\text { sample }\end{array}$ & -7.38 \\
\hline ECM-693 & $\begin{array}{l}\text { pumped } \\
\text { sample }\end{array}$ & -7.23 \\
\hline ECM-694 & $\begin{array}{l}\text { pumped } \\
\text { sample }\end{array}$ & -7.02 \\
\hline ECM-695 & $\begin{array}{l}\text { pumped } \\
\text { sample }\end{array}$ & -7.02 \\
\hline ECM-696 & $\begin{array}{l}\text { pumped } \\
\text { sample }\end{array}$ & -7.61 \\
\hline ECM-697 & pumped & -7.82 \\
\hline
\end{tabular}




\begin{tabular}{|c|c|c|}
\hline & sample & \\
\hline ECM-698 & borehole & -8.18 \\
\hline ECM-699 & borehole & -8.45 \\
\hline ECM-700 & borehole & -8 \\
\hline ECM-701 & borehole & -8.1 \\
\hline ECM-702 & borehole & -8.89 \\
\hline ECM-703 & borehole & -9.14 \\
\hline ECM-704 & spring & -8.5 \\
\hline ECM-705 & spring & -8.92 \\
\hline ECM-706 & borehole & -9.44 \\
\hline ECM-707 & borehole & -8.05 \\
\hline ECM-708 & borehole & -7.8 \\
\hline ECM-709 & borehole & -9.07 \\
\hline ECM-710 & borehole & -7.57 \\
\hline ECM-711 & borehole & -8.29 \\
\hline ECM-712 & borehole & -8.57 \\
\hline ECM-713 & borehole & -8.65 \\
\hline ECM-714 & spring & -9 \\
\hline ECM-715 & spring & -8.71 \\
\hline ECM-716 & borehole & -8.56 \\
\hline ECM-717 & borehole & -8.51 \\
\hline ECM-718 & borehole & -8.76 \\
\hline ECM-719 & spring & -8.29 \\
\hline ECM-720 & spring & -7.77 \\
\hline ECM-721 & borehole & -8.56 \\
\hline ECM-722 & borehole & -7.71 \\
\hline ECM-723 & borehole & -8.39 \\
\hline ECM-724 & borehole & -7.46 \\
\hline ECM-725 & spring & -8.46 \\
\hline ECM-726 & borehole & -7.61 \\
\hline ECM-727 & spring & -7.91 \\
\hline ECM-728 & borehole & -7.9 \\
\hline ECM-729 & spring & -7.7 \\
\hline ECM-730 & spring & -7.93 \\
\hline ECM-731 & spring & -7.75 \\
\hline ECM-732 & borehole & -7.27 \\
\hline ECM-733 & borehole & -8.06 \\
\hline ECM-734 & spring & -7.46 \\
\hline ECM-735 & spring & -8.14 \\
\hline ECM-736 & spring & -7.51 \\
\hline ECM-737 & borehole & -7.77 \\
\hline ECM-738 & spring & -8 \\
\hline ECM-739 & borehole & -8.03 \\
\hline ECM-740 & spring & -6.93 \\
\hline ECM-741 & borehole & -7.28 \\
\hline
\end{tabular}




\begin{tabular}{|c|c|c|}
\hline ECM-742 & borehole & -7.94 \\
\hline ECM-743 & borehole & -6.71 \\
\hline ECM-744 & borehole & -6.61 \\
\hline ECM-745 & borehole & -6.94 \\
\hline ECM-746 & borehole & -7.38 \\
\hline ECM-747 & borehole & -6.78 \\
\hline ECM-748 & borehole & -6.89 \\
\hline ECM-749 & borehole & -6.57 \\
\hline ECM-750 & borehole & -7.36 \\
\hline ECM-751 & borehole & -7.27 \\
\hline ECM-752 & borehole & -6.65 \\
\hline ECM-753 & borehole & -7.7 \\
\hline ECM-754 & borehole & -6.31 \\
\hline ECM-755 & borehole & -7.13 \\
\hline ECM-756 & borehole & -6.57 \\
\hline ECM-757 & borehole & -7.09 \\
\hline ECM-758 & borehole & -7.21 \\
\hline ECM-759 & shallow well & -7.01 \\
\hline ECM-760 & borehole & -5.44 \\
\hline ECM-761 & borehole & -7.4 \\
\hline ECM-762 & borehole & -6.4 \\
\hline ECM-763 & borehole & -6.89 \\
\hline ECM-764 & borehole & -6.39 \\
\hline ECM-765 & shallow well & -6.25 \\
\hline ECM-766 & borehole & -6.31 \\
\hline ECM-767 & borehole & -6.31 \\
\hline ECM-768 & borehole & -6.79 \\
\hline ECM-769 & borehole & -6.79 \\
\hline ECM-770 & borehole & -6.59 \\
\hline ECM-771 & borehole & -6.59 \\
\hline ECM-772 & borehole & -6.66 \\
\hline ECM-773 & borehole & -6.66 \\
\hline ECM-774 & borehole & -6.98 \\
\hline ECM-775 & borehole & -6.98 \\
\hline ECM-776 & borehole & -6.63 \\
\hline ECM-777 & borehole & -6.63 \\
\hline ECM-778 & borehole & -7.38 \\
\hline ECM-779 & borehole & -7.38 \\
\hline ECM-780 & borehole & -6.98 \\
\hline ECM-781 & borehole & -6.98 \\
\hline ECM-782 & spring & -7.8 \\
\hline ECM-783 & borehole & -8.11 \\
\hline ECM-784 & borehole & -8.39 \\
\hline ECM-785 & borehole & -8.17 \\
\hline ECM-786 & borehole & -8.97 \\
\hline
\end{tabular}




\begin{tabular}{|c|c|c|}
\hline ECM-787 & spring & -7.92 \\
\hline ECM-788 & borehole & -8.69 \\
\hline ECM-789 & borehole & -8.86 \\
\hline ECM-790 & borehole & -8.39 \\
\hline ECM-791 & borehole & -6.86 \\
\hline ECM-792 & borehole & -8.36 \\
\hline ECM-793 & borehole & -7.84 \\
\hline ECM-794 & shallow well & -7.18 \\
\hline ECM-795 & shallow well & -8.02 \\
\hline ECM-796 & borehole & -6.61 \\
\hline ECM-797 & borehole & -6.64 \\
\hline ECM-798 & borehole & -8.27 \\
\hline ECM-799 & borehole & -6.71 \\
\hline ECM-800 & spring & -6.43 \\
\hline ECM-801 & shallow well & -8.2 \\
\hline ECM-802 & spring & -8.8 \\
\hline ECM-803 & shallow well & -7.64 \\
\hline ECM-804 & borehole & -8.17 \\
\hline ECM-805 & borehole & -8.06 \\
\hline ECM-806 & shallow well & -7.32 \\
\hline ECM-807 & borehole & -7.69 \\
\hline ECM-808 & borehole & -8.18 \\
\hline ECM-809 & spring & -6.94 \\
\hline ECM-810 & spring & -7.3 \\
\hline ECM-811 & spring & -7.28 \\
\hline ECM-812 & spring & -7.44 \\
\hline ECM-813 & spring & -7.33 \\
\hline ECM-814 & spring & -7.35 \\
\hline ECM-815 & spring & -7.87 \\
\hline ECM-816 & spring & -7.28 \\
\hline ECM-817 & spring & -6.8 \\
\hline ECM-818 & spring & -6.82 \\
\hline ECM-819 & spring & -7.26 \\
\hline ECM-820 & spring & -7.16 \\
\hline ECM-821 & borehole & -8.34 \\
\hline ECM-822 & borehole & -8.83 \\
\hline ECM-823 & borehole & -7.43 \\
\hline ECM-824 & borehole & -8.29 \\
\hline ECM-825 & borehole & -8.79 \\
\hline ECM-826 & borehole & -8.37 \\
\hline ECM-827 & borehole & -8.1 \\
\hline ECM-828 & borehole & -8.31 \\
\hline ECM-829 & borehole & -8.62 \\
\hline ECM-830 & borehole & -8.04 \\
\hline ECM-831 & borehole & -8.48 \\
\hline
\end{tabular}




\begin{tabular}{|c|c|c|}
\hline ECM-832 & borehole & -8.15 \\
\hline ECM-833 & borehole & -7.78 \\
\hline ECM-834 & borehole & -7.25 \\
\hline ECM-835 & river & -7.49 \\
\hline ECM-836 & spring & -8.16 \\
\hline ECM-837 & borehole & -7.98 \\
\hline ECM-838 & borehole & -5.92 \\
\hline ECM-839 & borehole & -7.03 \\
\hline ECM-840 & borehole & -7 \\
\hline ECM-841 & borehole & -7.1 \\
\hline ECM-842 & borehole & -6.61 \\
\hline ECM-843 & borehole & -6.87 \\
\hline ECM-844 & borehole & -7.06 \\
\hline ECM-845 & borehole & -8.98 \\
\hline ECM-846 & borehole & -8.14 \\
\hline ECM-847 & borehole & -9.22 \\
\hline ECM-848 & borehole & -8.31 \\
\hline ECM-849 & borehole & -8.46 \\
\hline ECM-850 & borehole & -8.7 \\
\hline ECM-851 & borehole & -8.57 \\
\hline ECM-852 & borehole & -8.08 \\
\hline ECM-853 & borehole & -8.7 \\
\hline ECM-854 & borehole & -8.2 \\
\hline ECM-855 & borehole & -8.51 \\
\hline ECM-856 & borehole & -8.49 \\
\hline ECM-857 & borehole & -8.65 \\
\hline ECM-858 & borehole & -8.17 \\
\hline ECM-859 & borehole & -8.33 \\
\hline ECM-860 & borehole & -8.81 \\
\hline ECM-861 & borehole & -8.12 \\
\hline ECM-862 & borehole & -7.87 \\
\hline ECM-863 & borehole & -8.5 \\
\hline ECM-864 & borehole & -8.47 \\
\hline ECM-865 & borehole & -8.58 \\
\hline ECM-866 & borehole & -8.76 \\
\hline ECM-867 & borehole & -8.81 \\
\hline ECM-868 & borehole & -8.6 \\
\hline ECM-869 & borehole & -8.43 \\
\hline ECM-870 & borehole & -8.51 \\
\hline ECM-871 & borehole & -8.51 \\
\hline ECM-872 & borehole & -8.93 \\
\hline ECM-873 & borehole & -8.89 \\
\hline ECM-874 & borehole & -8.69 \\
\hline ECM-875 & borehole & -8.78 \\
\hline ECM-876 & borehole & -8.91 \\
\hline
\end{tabular}




\begin{tabular}{|c|c|c|}
\hline ECM-877 & borehole & -8.46 \\
\hline ECM-878 & borehole & -8.9 \\
\hline ECM-879 & borehole & -8.7 \\
\hline ECM-880 & spring & -7.25 \\
\hline ECM-881 & spring & -7.19 \\
\hline ECM-882 & spring & -7.41 \\
\hline ECM-883 & spring & -7.43 \\
\hline ECM-884 & spring & -7.58 \\
\hline ECM-885 & spring & -6.96 \\
\hline ECM-886 & spring & -7.25 \\
\hline ECM-887 & spring & -7.48 \\
\hline ECM-888 & spring & -7.52 \\
\hline ECM-889 & spring & -7.45 \\
\hline ECM-890 & borehole & -7.67 \\
\hline ECM-891 & borehole & -7.86 \\
\hline ECM-892 & borehole & -7.93 \\
\hline ECM-893 & borehole & -7.62 \\
\hline ECM-894 & borehole & -8.03 \\
\hline ECM-895 & spring & -7.38 \\
\hline ECM-896 & borehole & -8.95 \\
\hline ECM-897 & spring & -9.58 \\
\hline ECM-898 & spring & -9.7 \\
\hline ECM-899 & spring & -9.88 \\
\hline ECM-900 & spring & -9.62 \\
\hline ECM-901 & spring & -9.25 \\
\hline ECM-902 & spring & -9.67 \\
\hline ECM-903 & spring & -9.58 \\
\hline ECM-904 & spring & -9.96 \\
\hline ECM-905 & spring & -9.78 \\
\hline ECM-906 & spring & -9.43 \\
\hline ECM-907 & borehole & -7.96 \\
\hline ECM-908 & borehole & -7.68 \\
\hline ECM-909 & borehole & -8.34 \\
\hline ECM-910 & borehole & -8.13 \\
\hline ECM-911 & borehole & -8.28 \\
\hline ECM-912 & borehole & -8.22 \\
\hline ECM-913 & borehole & -7.87 \\
\hline ECM-914 & borehole & -7.47 \\
\hline ECM-915 & borehole & -7.86 \\
\hline ECM-916 & borehole & -9.35 \\
\hline ECM-917 & borehole & -7.2 \\
\hline ECM-918 & borehole & -7.85 \\
\hline ECM-919 & borehole & -8.49 \\
\hline ECM-920 & borehole & -7.57 \\
\hline ECM-921 & borehole & -7.88 \\
\hline
\end{tabular}




\begin{tabular}{|c|c|c|}
\hline ECM-922 & spring & -7.9 \\
\hline ECM-923 & borehole & -7.89 \\
\hline ECM-924 & borehole & -6.95 \\
\hline ECM-925 & borehole & -7.12 \\
\hline ECM-926 & borehole & -7.43 \\
\hline ECM-927 & borehole & -6.88 \\
\hline ECM-928 & borehole & -7.03 \\
\hline ECM-929 & borehole & -6.8 \\
\hline ECM-930 & borehole & -6.7 \\
\hline ECM-931 & borehole & -6.9 \\
\hline ECM-932 & borehole & -6.7 \\
\hline ECM-933 & borehole & -7.31 \\
\hline ECM-934 & borehole & -6.98 \\
\hline ECM-935 & borehole & -7.19 \\
\hline ECM-936 & borehole & -7.37 \\
\hline ECM-937 & borehole & -8.17 \\
\hline ECM-938 & spring & -9.39 \\
\hline ECM-939 & spring & -9.62 \\
\hline ECM-940 & borehole & -7.95 \\
\hline ECM-941 & borehole & -8.3 \\
\hline ECM-942 & borehole & -8.02 \\
\hline ECM-943 & borehole & -8.51 \\
\hline ECM-944 & borehole & -8.18 \\
\hline ECM-945 & borehole & -8.09 \\
\hline ECM-946 & borehole & -8.1 \\
\hline ECM-947 & borehole & -8.2 \\
\hline ECM-948 & borehole & -8.25 \\
\hline ECM-949 & borehole & -8.2 \\
\hline ECM-950 & borehole & -7.75 \\
\hline ECM-951 & borehole & -8.25 \\
\hline ECM-952 & borehole & -8.44 \\
\hline ECM-953 & borehole & -8.04 \\
\hline ECM-954 & borehole & -8.1 \\
\hline ECM-955 & borehole & -7.21 \\
\hline ECM-956 & borehole & -7.36 \\
\hline ECM-957 & borehole & -7.27 \\
\hline ECM-958 & borehole & -7.28 \\
\hline ECM-959 & borehole & -7.35 \\
\hline ECM-960 & borehole & -8.17 \\
\hline ECM-961 & borehole & -7.53 \\
\hline ECM-962 & borehole & -7.11 \\
\hline ECM-963 & borehole & -6.35 \\
\hline ECM-964 & borehole & -7.51 \\
\hline ECM-965 & borehole & -7.08 \\
\hline ECM-966 & borehole & -7.25 \\
\hline
\end{tabular}




\begin{tabular}{|l|l|l|} 
ECM-967 & borehole & -7.1 \\
\hline ECM-968 & borehole & -6.96 \\
\hline ECM-969 & borehole & -7.39 \\
\hline ECM-970 & borehole & -7.33 \\
\hline ECM-971 & borehole & -7.19 \\
\hline ECM-972 & borehole & -7.13 \\
\hline ECM-973 & borehole & -7.1 \\
\hline ECM-974 & borehole & -7.5 \\
\hline ECM-975 & borehole & -8.16 \\
\hline
\end{tabular}


Table 1

\begin{tabular}{|c|c|c|c|c|c|c|}
\hline \multicolumn{7}{|c|}{ Drinking water ranges } \\
\hline & & -4 to -5 & -5 to -6 & -6 to -7 & -7 to -8 & -8 to -9 \\
\hline${ }^{87} \mathrm{Sr} /{ }^{86} \mathrm{Sr}$ ranges & $\begin{array}{l}\text { \% } \\
\text { coverage }\end{array}$ & $1 \%$ & $8 \%$ & $21 \%$ & $60 \%$ & $11 \%$ \\
\hline .704 to .708 & $0.90 \%$ & $0.01 \%$ & $0.07 \%$ & $0.19 \%$ & $0.54 \%$ & $0.10 \%$ \\
\hline .708 to .709 & $14.00 \%$ & $0.10 \%$ & $1.12 \%$ & $2.88 \%$ & $8.36 \%$ & $1.55 \%$ \\
\hline $.709-.710$ & $39.00 \%$ & $0.27 \%$ & $3.12 \%$ & $8.03 \%$ & $23.28 \%$ & $4.33 \%$ \\
\hline $.710-.711$ & $2.00 \%$ & $0.01 \%$ & $0.16 \%$ & $0.41 \%$ & $1.19 \%$ & $0.22 \%$ \\
\hline $.711-.712$ & $19.00 \%$ & $0.13 \%$ & $1.52 \%$ & $3.91 \%$ & $11.34 \%$ & $2.11 \%$ \\
\hline $.712-.713$ & $17.00 \%$ & $0.12 \%$ & $1.36 \%$ & $3.50 \%$ & $10.15 \%$ & $1.89 \%$ \\
\hline $.713-.72$ & $8.00 \%$ & $0.06 \%$ & $0.64 \%$ & $1.65 \%$ & $4.78 \%$ & $0.89 \%$ \\
\hline$>.72$ & $0.30 \%$ & $0.00 \%$ & $0.02 \%$ & $0.06 \%$ & $0.18 \%$ & $0.03 \%$ \\
\hline
\end{tabular}




\section{Figures and captions}

Figure 1 An outline of Great Britain showing the location of the archaeological sites from which data is presented. Highlighted sites are those used to define the two subsets: low and high rainfall areas.

Figure 2 A scatter diagram of strontium concentrations (ppm) plotted against ${ }^{87} \mathrm{Sr} /{ }^{86} \mathrm{Sr}$ isotope ratio for all British burials given in Appendix 1. A

Figure 3 Boxplot comparison of the strontium isotope composition of tooth enamel from Britain compared with biosphere data from Britain ${ }^{29}$.

Figure 4 An histogram of the best estimate for the distribution of oxygen isotope composition in tooth enamel from Britain excluding 51 individuals deemed unlikely to be of British origin (see main text.).

Figure 5 An histogram of shallow ground, and well water, oxygen isotope composition from Britain. (see Appendix 2)

Figure 6 A boxplot comparison of drinking water values derived from tooth enamel with directly measured values from drinking water sources, both from Britain .

Figure 7 A comparison of $\delta^{18} \mathrm{O}_{\text {(VSMOw) }}$ in teeth, that formed at different ages, from (7a) 3 teeth from each of 20 individuals ${ }^{44}$ and (7b) a comparison of all the data from differing tooth types from across Britain.

Figure 7 A Comparison of $\delta^{18} \mathrm{O}_{\text {VsMOw }}$ tooth enamel data from different periods. It should be noted that these groups do not all sample the same geographic distributions.

Figure 9 A comparison of normal distributions of oxygen isotope composition of human tooth enamel from British sites where burial assemblages are taken to be predominantly of local origin.

Figure 10 A comparison of the population means (95\% CI) of tooth enamel oxygen isotope composition from a Roman site at Catterick, Yorkshire and four individuals from an Anglo Saxon site at Easington, on the east coast of Yorkshire compared with the control data sets for the leeward and windward populations. 
Figure 11 A probability density curve for oxygen isotope composition from Roman period burials at the Lankhills cemetery near Winchester. The curve is constructed allocating a blanket error of $\pm 0.15 \%$ o to all oxygen isotope analyses.

Figure 12 A map of the Roman Empire (excluding Britain) contoured for rainwater composition derived from GNIP data and including a tabulation of the percentage areas assonated with different water compositions. .

Figure 13. Comparison of the population means and 95\% confidence intervals of tooth enamel oxygen isotope composition from the Amesbury Archer and Companion against the defining population datasets for high and low rainfall areas.

Table 1 Mathematically combined spatial probability of strontium and oxygen isotope combinations across Britain derived from strontium biosphere ${ }^{\text {ref }}$ and surface and spring water maps $45^{\circ}$

Appendix 1 Strontium and oxygen isotope composition for all data discussed in this paper. Samples in italics are those excluded from the histogram given in Figure 4.

Appendix 2. Oxygen isotope composition of shallow ground water and well water extracted from the British Geological Survey database. The data are tabulated in three parallel sets for compactness and give the type of water source and the measured $\delta^{18} \mathrm{O}_{(\mathrm{VMOW})}$ value. Further details can be obtained from BGS. 


\begin{tabular}{|c|c|c|c|c|c|c|}
\hline & & $:-4$ to -5 & $:-5$ to -6 & $:-6$ to -7 & $:-7$ to -8 & $:-8$ to -9 \\
\hline \multicolumn{2}{|c|}{ strontium isotope range } & $1 \%$ & $8 \%$ & $21 \%$ & $60 \%$ & $11 \%$ \\
\hline .704 to .708 & $0.9 \%$ & $0.01 \%$ & $0.07 \%$ & $0.187 \%$ & $0.54327 \%$ & $0.101 \%$ \\
\hline .708 to .709 & $14.0 \%$ & $0.10 \%$ & $1.12 \%$ & $2.88 \%$ & $8.36 \%$ & $1.55 \%$ \\
\hline $.709-.710$ & $39.0 \%$ & $0.27 \%$ & $3.12 \%$ & $8.03 \%$ & $23.28 \%$ & $4.33 \%$ \\
\hline $.710-.711$ & $2.0 \%$ & $0.01 \%$ & $0.16 \%$ & $0.41 \%$ & $1.19 \%$ & $0.22 \%$ \\
\hline $.711-.712$ & $19.0 \%$ & $0.13 \%$ & $1.52 \%$ & $3.91 \%$ & $11.34 \%$ & $2.11 \%$ \\
\hline $.712-.713$ & $17.0 \%$ & $0.12 \%$ & $1.36 \%$ & $3.50 \%$ & $10.15 \%$ & $1.89 \%$ \\
\hline $.713-.72$ & $8.0 \%$ & $0.06 \%$ & $0.64 \%$ & $1.65 \%$ & $4.78 \%$ & $0.89 \%$ \\
\hline$>.72$ & $0.3 \%$ & $0.00 \%$ & $0.02 \%$ & $0.06 \%$ & $0.18 \%$ & $0.03 \%$ \\
\hline
\end{tabular}


